\title{
Melting hadrons, boiling quarks
}

\author{
Johann Rafelski ${ }^{1,2, a}$ \\ 1 CERN-PH/TH, 1211 Geneva 23, Switzerland \\ 2 Department of Physics, The University of Arizona Tucson, Arizona, 85721, USA
}

Received: 12 August 2015 / Revised: 23 August 2015

Published online: 22 September 2015

(C) The Author(s) 2015. This article is published with open access at Springerlink.com Communicated by T.S. Bíró

\begin{abstract}
In the context of the Hagedorn temperature half-centenary I describe our understanding of the hot phases of hadronic matter both below and above the Hagedorn temperature. The first part of the review addresses many frequently posed questions about properties of hadronic matter in different phases, phase transition and the exploration of quark-gluon plasma (QGP). The historical context of the discovery of QGP is shown and the role of strangeness and strange antibaryon signature of QGP illustrated. In the second part I discuss the corresponding theoretical ideas and show how experimental results can be used to describe the properties of QGP at hadronization. The material of this review is complemented by two early and unpublished reports containing the prediction of the different forms of hadron matter, and of the formation of QGP in relativistic heavy ion collisions, including the discussion of strangeness, and in particular strange antibaryon signature of QGP.
\end{abstract}

\section{Introduction}

The years 1964/65 saw the rise of several new ideas which in the following 50 years shaped the discoveries in fundamental subatomic physics:

1) The Hagedorn temperature $T_{\mathrm{H}}$; later recognized as the melting point of hadrons into

2) Quarks as building blocks of hadrons; and

3) The Higgs particle and field escape from the Goldstone theorem, allowing the understanding of weak interactions, the source of inertial mass of the elementary particles.

The topic in this paper is Hagedorn temperature $T_{\mathrm{H}}$ and the strong interaction phenomena near to $T_{\mathrm{H}}$. I present an overview of 50 years of effort with emphasis on:

a) Hot nuclear and hadronic matter.

b) Critical behavior near $T_{\mathrm{H}}$.

c) Quark-gluon plasma (QGP).

d) Relativistic heavy ion (RHI) collisions $^{1}$.

e) The hadronization process of QGP.

f) Abundant production of strangeness flavor.

\footnotetext{
${ }^{a}$ e-mail: rafelski@physics.arizona.edu

1 We refer to atomic nuclei which are heavier than the $\alpha$ particle as "heavy ions".
}

This presentation connects and extends a recent retrospective work, ref. [1]: Melting Hadrons, Boiling Quarks; From Hagedorn temperature to ultra-relativistic heavy-ion collisions at CERN; with a tribute to Rolf Hagedorn. This report complements prior summaries of our work: 1986 [2], 1991 [3],1996 [4], 2000 [5], 2002 [6], 2008 [7].

A report on "Melting Hadrons, Boiling Quarks and $T_{\mathrm{H}}$ " relates strongly to quantum chromodynamics (QCD), the theory of quarks and gluons, the building blocks of hadrons, and its lattice numerical solutions; QCD is the quantum (Q) theory of color-charged (C) quark and gluon dynamics (D); for numerical study the space-time continuum is discretized on a "lattice".

Telling the story of how we learned that strong interactions are a gauge theory involving two types of particles, quarks and gluons, and the working of the lattice numerical method would entirely change the contents of this article, and be beyond the expertise of the author. I recommend instead the book by Weinberg [8], which also shows the historical path to QCD. The best sources of the QCD relation to the topic of this article are: a) the book by Kohsuke Yagi and Tetsuo Hatsuda [9] as well as, b) the now 15 year old monograph by Letessier and the author [6]. We often refer to lattice-QCD method to present QCD properties of interest in this article. There are books and many reviews on lattice implementation of gauge theories of interacting fields, also specific to hot-lattice-QCD method. At the time of writing I do not have a favorite to recommend. 
Immediately in the following subsect. 1.1 the famous Why? is addressed. After that I turn to answering the How? question in subsect. 1.2, and include a few reminiscences about the accelerator race in subsect. 1.3. I close this introduction with subsect. 1.4 where the organization and contents of this review will be explained.

\subsection{What are the conceptual challenges of the $\mathrm{QGP} / \mathrm{RHI}$ collisions research program?}

Our conviction that we achieved in laboratory experiments the conditions required for melting (we can also say, dissolution) of hadrons into a soup of boiling quarks and gluons became firmer in the past 15-20 years. Now we can ask, what are the "applications" of the quark-gluon plasma physics? Here is a short wish list:

1) Nucleons dominate the mass of matter by a factor 1000. The mass of the three "elementary" quarks found in nucleons is about 50 times smaller than the nucleon mass. Whatever compresses and keeps the quarks within the nucleon volume is thus the source of nearly all of mass of matter. This clarifies that the Higgs field provides the mass scale to all particles that we view today as elementary. Therefore only a small \%-sized fraction of the mass of matter originates directly in the Higgs field; see sect. 7.1 for further discussion. The question: What is mass? can be studied by melting hadrons into quarks in RHI collisions.

2) Quarks are kept inside hadrons by the "vacuum" properties which abhor the color charge of quarks. This explanation of 1) means that there must be at least two different forms of the modern æether that we call "vacuum": the world around us, and the holes in it that are called hadrons. The question: Can we form arbitrarily big holes filled with almost free quarks and gluons? was and remains the existential issue for laboratory study of hot matter made of quarks and gluons, the QGP. Aficionados of the lattice-QCD should take note that the presentation of two phases of matter in numerical simulations does not answer this question as the lattice method studies the entire Universe, showing hadron properties at low temperature, and QGP properties at high temperature.

3) We all agree that QGP was the primordial BigBang stuff that filled the Universe before "normal" matter formed. Thus any laboratory exploration of the QGP properties solidifies our models of the Big Bang and allows us to ask these questions: What are the properties of the primordial matter content of the Universe? and How does "normal" matter formation in early Universe work?

4) What is flavor? In elementary particle collisions, we deal with a few, and in most cases only one, pair of newly created 2nd, or 3rd flavor family of particles at a time. A new situation arises in the QGP formed in relativistic heavy ion collisions. QGP includes a large number of particles from the second family: the strange quarks and also, the yet heavier charmed quarks; and from the third family at the LHC we expect an appreciable abundance of bottom quarks. The novel ability to study a large number of these 2nd and 3rd generation particles offers a new opportunity to approach in an experiment the riddle of flavor.
5) In relativistic heavy ion collisions the kinetic energy of ions feeds the growth of quark population. These quarks ultimately turn into final state material particles. This means that we study experimentally the mechanisms leading to the conversion of the colliding ion kinetic energy into mass of matter. One can wonder aloud if this sheds some light on the reverse process: Is it possible to convert matter into energy in the laboratory?

The last two points show the potential of "applications" of QGP physics to change both our understanding of, and our place in the world. For the present we keep these questions in mind. This review will address all the other challenges listed under points 1),2), and 3) above; however, see also thoughts along comparable foundational lines presented in subsects. 7.3 and 7.4.

\subsection{From melting hadrons to boiling quarks}

With the hindsight of 50 years I believe that Hagedorn's effort to interpret particle multiplicity data has led to the recognition of the opportunity to study quark deconfinement at high temperature. This is the topic of the book [1] Melting Hadrons, Boiling Quarks; From Hagedorn temperature to ultra-relativistic heavy-ion collisions at CERN; with a tribute to Rolf Hagedorn published at Springer Open, i.e. available for free on-line. This article should be seen as a companion addressing more recent developments, and setting a contemporary context for this book.

How did we get here? There were two critical milestones:

I) The first milestone occurred in 1964-1965, when Hagedorn, working to resolve discrepancies of the statistical particle production model with the $p p$ reaction data, produced his "distinguishable particles" insight. Due to a twist of history, the initial research work was archived without publication and has only become available to a wider public recently; that is, 50 years later, see chapt. 19 in [1] and ref. [10]. Hagedorn went on to interpret the observation he made. Within a few months, in Fall 1964, he created the Statistical Bootstrap Model (SBM) [11], showing how the large diversity of strongly interacting particles could arise; Steven Frautschi [12] coined in 1971 the name "Statistical Bootstrap Model".

II) The second milestone occurred in the late $70 \mathrm{~s}$ and early 80s when we spearheaded the development of an experimental program to study "melted" hadrons and the "boiling" quark-gluon plasma phase of matter. The intense theoretical and experimental work on the thermal properties of strongly interacting matter, and the confirmation of a new quark-gluon plasma paradigm started in 1977 when the SBM mutated to become a model for melting nuclear matter. This development motivated the experimental exploration in the collisions of heavy nuclei at relativistic energies of the phases of matter in conditions close to those last seen in the early Universe. I refer to Hagedorn's account of these developments for further details chapt. 25 loc. cit. and ref. [13]. We return to this time period in subsect. 4.1 . 
At the beginning of this new field of research in the late 70s, quark confinement was a mystery for many of my colleagues; gluons mediating the strong color force were neither discovered nor widely accepted, especially not among my nuclear physics peers, and QCD vacuum structure was just finishing kindergarten. The discussion of a new phase of deconfined quark-gluon matter was therefore in many eyes not consistent with established wisdom and certainly too ambitious for the time.

Similarly, the special situation of Hagedorn deserves remembering: early on Hagedorn's research was undermined by outright personal hostility; how could Hagedorn dare to introduce thermal physics into the field governed by particles and fields? However, one should also take note of the spirit of academic tolerance at CERN. Hagedorn advanced through the ranks along with his critics, and his presence attracted other like-minded researchers, who were welcome in the CERN Theory Division, creating a bridgehead towards the new field of RHI collisions when the opportunity appeared on the horizon.

In those days, the field of RHI collisions was in other ways rocky terrain:

1) RHI collisions required the use of atomic nuclei at highest energy. This required cooperation between experimental nuclear and particle physicists. Their culture, background, and experience differed. A similar situation prevailed within the domain of theoretical physics, where an interdisciplinary program of research merging the three traditional physics domains had to form. While ideas of thermal and statistical physics needed to be applied, very few subatomic physicists, who usually deal with individual particles, were prepared to deal with many body questions. There were also several practical issues: In which (particle, nuclear, stat-phys) journal can one publish and who could be the reviewers (other than direct competitors)? To whom to apply for funding? Which conference to contribute to?

2) The small group of scientists who practiced RHI collisions were divided on many important questions. In regard to what happens in relativistic collision of nuclei the situation was most articulate: a) One group believed that nuclei (baryons) pass through each other with a new phase of matter formed in a somewhat heated projectile and/or target. This picture required detection systems of very different character than the systems required by, both: b) those who believed that in RHI collisions energy would be consumed by a shock wave compression of nuclear matter crashing into the center of momentum frame; and c) a third group who argued that up to top CERN$\operatorname{SPS}\left(\sqrt{\left(s_{N N}\right)} \simeq \mathcal{O}(20) \mathrm{GeV}\right)$ collision energy a high temperature, relatively low baryon density quark matter fireball will be formed. The last case turned out to be closest to results obtained at CERN-SPS and at Brookhaven National Laboratory (BNL) RHI Collider (RHIC).

From outside, we were ridiculed as being speculative; from within we were in state of uncertainty about the fate of colliding matter and the kinetic energy it carried, with disagreements that ranged across theory vs. experiment, and particle vs. nuclear physics. In this situation, "QGP formation in RHI collisions" was a field of research that could have easily fizzled out. However, in Europe there was CERN, and in the US there was strong institutional support. Early on it was realized that RHI collisions required large experiments uniting much more human expertise and manpower as compared to the prior nuclear and even some particle physics projects. Thus work had to be centralized in a few "pan-continental" facilities. This meant that expertise from a few laboratories would need to be united in a third location where prior investments would help limit the preparation time and cost.

\subsection{The accelerator race for quark matter}

These considerations meant that in Europe the QGP formation in RHI collisions research program found its home at CERN. The CERN site benefited from being a multiaccelerator laboratory with a large pool of engineering expertise and where some of the necessary experimental equipment already existed, thanks to prior related particle physics efforts.

The CERN program took off by the late $80 \mathrm{~s}$. The time line of the many CERN RHI experiments through the beginning of this millennium is shown in fig. 1; the representation is based on a similar CERN document from the year 2000. The experiments WA85, NA35, HELIOS2, NA38 were built largely from instrumental components from prior particle physics detectors. Other experiments and/or experimental components were contributed by US and European laboratories. These include the heavy ion source and its preaccelerator complex, required for heavy ion insertion into CERN beam lines.

When the CERN SPS program faded out early in this millennium, the resources were focused on the LHC-ion collider operations, and in the US, the RHIC came on-line. As this is written, the SPS fixed target program experiences a second life; the experiment NA61, built with large input from the NA49 equipment, is searching for the onset of QGP formation, see subsect. 6.3.

The success of the SPS research program at CERN has strongly supported the continuation of the RHI collision program. The Large Hadron Collider (LHC) was designed to accept highest energy counter propagating heavy ion beams opening the study of a new domain of collision energy. LHC-ion operation allows us to exceed the top RHIC energy range by more than an order of magnitude. In preparation for LHC-ion operations, in the mid-90s the SPS groups founded a new collider collaboration, and have built one of the four LHC experiments dedicated to the study of RHI collisions. Two other experiments also participate in the LHC-ion research program which we will introduce in subsect. 6.2.

In parallel to CERN there was a decisive move in the same direction in the US. The roots of the US relativistic heavy ion program predate the interest of CERN by nearly a decade. In 1975, the Berkeley SuperHILAC, a low energy heavy ion accelerator was linked to the Bevatron, an antique particle accelerator at the time, yet capable of accelerating the injected ions to relativistic energies with 


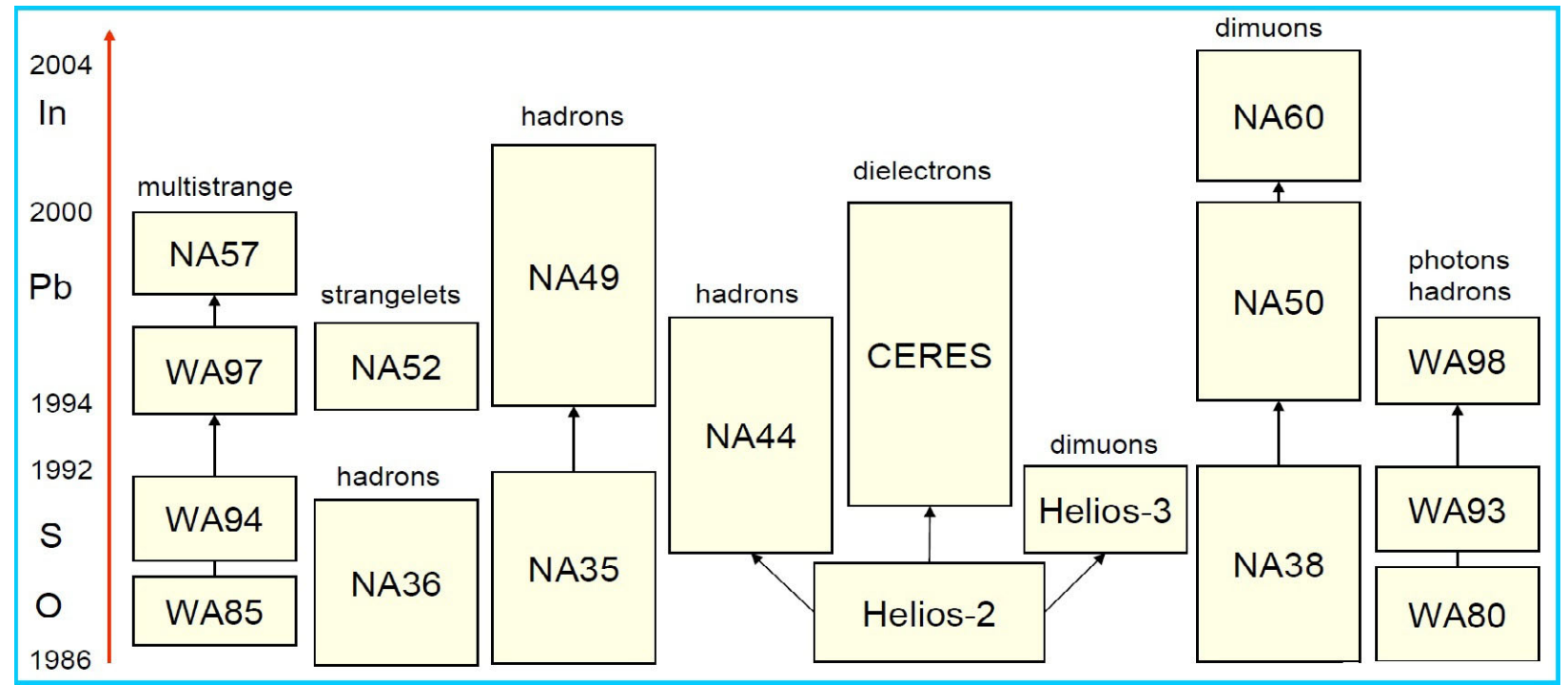

Fig. 1. Time line of the CERN-SPS RHI program: on the left axis we see year and ion beam available $(\mathrm{S}=\mathrm{sulfur}, \mathrm{Pb}=\mathrm{lead}$, $\mathrm{In}=$ indium) as a "function" of the experimental code. The primary observables are indicated next to each square; arrows connecting the squares indicate that the prior equipment and group, both in updated format, continued. Source: CERN release February 2000 modified by the author.

the Lorentz factor above two. The system of accelerators was called the Bevalac. It offered beams of ions which were used in study of properties of compressed nuclear matter, conditions believed to be similar to those seen within collapsing neutron stars.

As interest in the study of quark matter grew, by 1980 the Bevalac scientists formulated the future Variable Energy Nuclear Synchrotron (VENUS) heavy ion facility. Representing the Heavy Ion Program at Berkeley Howell Pugh [14] opened in October 1980 the "Quark Matter 1 " conference at GSI in Germany making this comment

\section{"20 A GeV $<E<1000 A \mathrm{GeV} \ldots$. LBL's VENUS proposal. In view of the long lead time in VENUS construction it would be extremely valuable to pro- ceed with the necessary modifications to accelerate light nuclei at CERN. . . the rich environment of so- phisticated detectors would be hard to reproduce elsewhere."}

It is clear from the context that CERN was in these remarks synonymous with CERN-ISR, a collider. Within following two years the incoming CERN Director Herwig Schopper closed ISR and created an alternative, the SPS heavy ion program capable of using the heaviest ions.

However, Pugh's remarks created in the minds of all concerned in the US a question: was there a place in the US, other than LBL, with capabilities similar to CERN? When Berkley moved to define the research program for an ultrarelativistic heavy ion collider in 1983, another candidate laboratory was waiting in the wings: The Brookhaven National Laboratory (BNL) had a completed project with 4 experimental halls. This was to be the $p p$ collider ISABELLE, now mothballed having been scooped by CERN's bet on the Spp $\overline{\mathrm{S}}$ collider in the race to discover the $\mathrm{W}$ and $\mathrm{Z}$ weak interaction mesons. If ISABELLE were modified to be a RHI Collider (RHIC), it was thought that it could be completed within a few years, offering the US a capability comparable to that expected by Pugh at CERN.

This evaluation prompted a major investment decision by the US Department of Energy to create a new relativistic heavy ion research center at BNL shown in fig. 2, a plan that would be cementing the US leadership role in the field of heavy ions. In a first step, already existing tandems able to create low energy heavy ion beams were connected by a transfer line to the already existing AGS proton synchrotron adapted to accelerate these ions. In this step a system similar to the Berkeley Bevalac was formed, while beam energies about 7 times greater than those seen at Bevalac could be reached.

The AGS-ion system performed experiments with fixed targets, serving as a training ground for the next generation of experimentalists. During this time, another transfer line was built connecting AGS to the ISABELLE project tunnel, in which the RHIC was installed. The initial RHIC experiments are shown around their ring locations in fig. 2: STAR, PHENIX, PHOBOS and BRAHMS, see also subsect. 4.2. The first data taking at RHIC began in Summer 2001, about 10 years later than many had hoped for back in 1984.

I recall vividly that when in 1984 we were told at a meeting at BNL that RHIC was to operate by 1990, a colleague working at the Bevalac asked, why not 1988 ? So a big question remains today: why in the end was it 2001? In seeking an answer we should note that while the RHIC project took 17 years to travel from the first decision to first beam, SPS took 11 years ( $\mathrm{Pb}$ beam capability). However, SPS was an already built, functional accelerator. Moreover, RHIC development was hindered by the need to move heavy ion activities from LBL to 


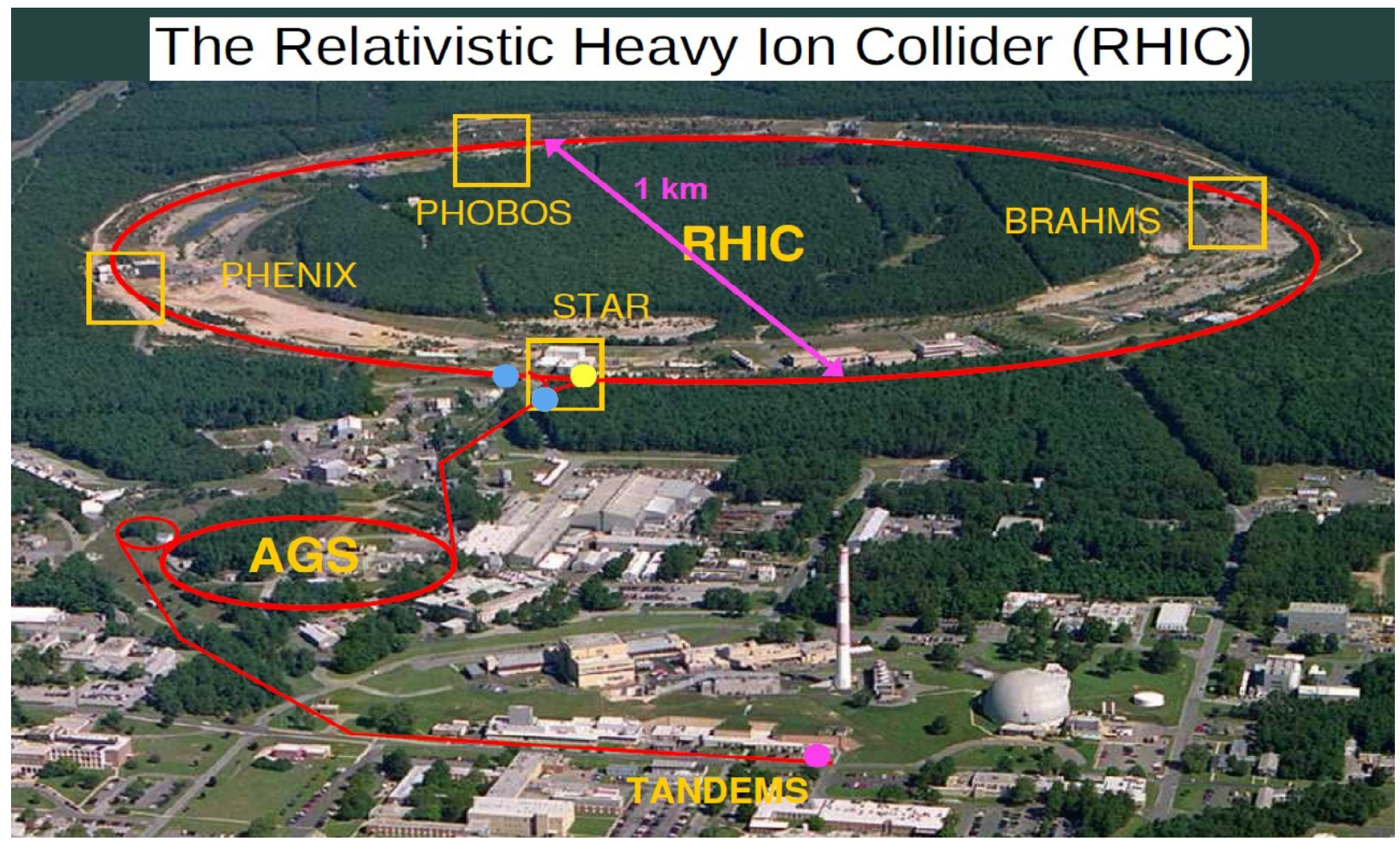

Fig. 2. The Brookhaven National Laboratory heavy ion accelerator complex. Creative Commons picture modified by the author.

BNL, by the adaptation of ISABELLE design to fit RHIC needs, and by typical funding constraints. As this work progressed nobody rushed. I think this was so since at BNL the opinion prevailed that RHIC was invulnerable, a dream machine not to be beaten in the race to discover the new phase of matter. Hereto I note that nobody back then could tell what the energy threshold for QGP formation in the very heavy ion collisions would be. The theoretical presumption that this threshold was above the energy produced at SPS turned out to be false.

Because data taking for the RHIC beam did not happen until 2001, the priority in the field of heavy ions that the US pioneered in a decisive way at Berkeley in the early 70s passed on to CERN where a large experimental program at SPS was developed, and as it is clear today, the energy threshold for QGP formation in $\mathrm{Pb}-\mathrm{Pb}$ collisions was within SPS reach, see sect. 4.2. It is important to remember that CERN moved on to develop the relativistic heavy ion research program under the leadership of Herwig Schopper. Schopper, against great odds, bet his reputation on Heavy Ions to become one of the pillars of CERN's future. This decision was strongly supported by many national nuclear physics laboratories in Europe, where in my opinion the most important was the support offered by the GSI and the continued development of relativistic heavy ion physics by one of GSI directors, Rudolph Bock.

To conclude the remarks about where we came from and where we are now: a new fundamental set of science arguments about the formation of quark-gluon plasma and deep-rooted institutional support carried the field forward.
CERN was in a unique position to embark on RHI research by having not only the accelerators, engineering expertise, and research equipment, but mainly due to Hagedorn, also the scientific expertise on the ground, for more detail consult ref. [1]. In the US a major new experimental facility, RHIC at BNL, was developed. With the construction of LHC at CERN a new RHI collision energy domain was opened. The experimental programs at SPS, RHIC and LHC-ion continue today.

\subsection{Format of this review}

More than 35 years into the QGP endeavor I can say with conviction that the majority of nuclear and particle physicists and the near totality of the large sub-group directly involved with the relativistic heavy ion collision research agree that a new form of matter, the (deconfined) quarkgluon plasma phase has been discovered. The discovery announced at CERN in the year 2000, see subsect. 4.2, has been confirmed both at RHIC and by the recent results obtained at LHC. This review has, therefore, as its primary objective, the presentation of the part of this announcement that lives on, see subsect. 4.3, and how more recent results are addressing these questions: What are the properties of hot hadron matter? How does it turn into QGP, and how does QGP turn back into normal matter? These are to be the topics addressed in the second half of this review.

There are literally thousands of research papers in this field today; thus this report cannot aim to be inclusive of all work in the field. We follow the example of John 
A. Wheeler. Addressing in his late age a large audience of physicists, he showed one transparency with one line, "What is the question?". In this spirit, this review begins with a series of questions, and answers, aiming to find the answer to: Which question is THE question today? A few issues we raise are truly fundamental present day challenges. Many provide an opportunity to recognize the state of the art, both in theory and experiment. Some questions are historical in character and will kick off a debate with other witnesses with a different set of personal memories.

These introductory questions are grouped into three separate sections: first come the theoretical concepts on the hadron side of hot hadronic matter, sect. 2 ; next, concepts on the quark side, sect. 3 ; and third, the experimental "side" sect. 4 about RHI collisions. Some of the questions formulated in sects. 2, 3, and 4 introduce topics that this review addresses in later sections in depth. The roles of strangeness enhancement and strange antibaryon signature of QGP are highlighted.

We follow this discussion by addressing the near future of the QGP and RHI collision research in the context of this review centered around the strong interactions and hadron-quarks phase. In sect. 5, I present several conceptual RHI topics that both are under present active study, and which will help determine which direction the field will move on in the coming decade. Section 6 shows the current experimental research program that address these questions. Assuming that this effort is successful, I propose in sect. 7 the next generation of physics challenges. The topics discussed are very subjective; other authors will certainly see other directions and propose other challenges of their interest.

In sect. 8 we deepen the discussion of the origins and the contents of the theoretical ideas that have led Hagedorn to invent the theoretical foundations leading on to $T_{\mathrm{H}}$ and melting hadrons. The technical discussion is brief and serves as an introduction to ref. [15] which is published for the first time as an addendum to this review. Section 8 ends with a discussion, subsect. 8.5, of how the present day lattice-QCD studies test and verify the theory of hot nuclear matter based on SBM.

Selected theoretical topics related to the study of QGP hadronization are introduced in the following: In sect. 9 we describe the numerical analysis tool within the Statistical Hadronization Model (SHM); that is, the SHARE suite of computer programs and its parameters. We introduce practical items such as triggered centrality events and rapidity volume $\mathrm{d} V / \mathrm{d} y$, resonance decays, particle number fluctuations, which all enter into the RHIC and LHC data analysis.

Section 10 presents the results of the SHM analysis with emphasis put on bulk properties of the fireball; subsect. 10.1, addresses SPS and RHIC prior to LHC, while in subsect. 10.2: it is shown how hadron production can be used to determine the properties of QGP and how the threshold energy for QGP formation is determined. The results of RHIC and LHC are compared and the universality of QGP hadronization across a vast range of energy and fireball sizes described. Subsection 10.3 explains, in terms of evaluation by example of prior work, why the prior two subsections address solely the SHARE-model results. In subsect. 10.4 the relevance of LHC results to QGP physics is described, and further lattice-QCD relations to these results pointed out.

The final sect. 11 does not attempt a summary which in case of a review would mean presenting a review of a review. Instead, a few characteristic objectives and results of this review are highlighted.

An integral part of this review are two previously unpublished technical papers which are for the first time in print as an addendum to this review, one from 1980 (ref. [15]) and another from 1983 (ref. [16]). These two are just a tip of an iceberg; there are many other unpublished papers by many authors hidden in conference volumes. There is already a published work reprint volume [17] in which the pivotal works describing QGP theoretical foundations are reproduced; however, the much less accessible and often equally interesting unpublished work is at this juncture in time practically out of sight. This was one of the reasons leading to the presentation of ref. [1]. These two papers were selected from this volume and are shown here unabridged. They best complement the contents of this review, providing technical detail not repeated here, while also offering a historical perspective. Beside the key results and/or discussion they also show the rapid shift in the understanding that manifested itself within a short span of two years.

Reference [15] presents Extreme States of Nuclear Matter, from the Workshop on Future Relativistic Heavy Ion Experiments held 7-10 October 1980. This small gathering convened by Rudolph Bock and Reinhard Stock is now considered to be the first of the "Quark Matter" series i.e. QM80 conference. Most of this report is a summary of the theory of hot hadron gas based on Hagedorn's Statistical Bootstrap Model (SBM). The key new insight in this work was that in RHI collisions the production of particles rather than the compression of existent matter was the determining factor. The hadron gas phase study was complemented by a detailed QGP model presented as a large, hot, interacting quark-gluon bag. The phase boundary between these two phases characterized by Hagedorn temperature $T_{\mathrm{H}}$ was evaluated in quantitative manner. It was shown how the consideration of different collision energies allows us to explore the phase boundary. This 1980 paper ends with the description of strangeness flavor as the observable of QGP. Strange antibaryons are introduced as a signature of quark-gluon plasma.

Reference [16] presents Strangeness and Phase Changes in Hot Hadronic Matter, from the Sixth High Energy Heavy Ion Study, Berkeley, 28 June - 1 July 1983. The meeting, which had a strong QGP scientific component, played an important role in the plans to develop a dedicated relativistic heavy ion collider (RHIC). In this lecture I summarize and update in qualitative terms the technical phase transition consideration seen in ref. [15], before turning to the physics of strangeness in hot hadron and quark matter. The process of strangeness production 


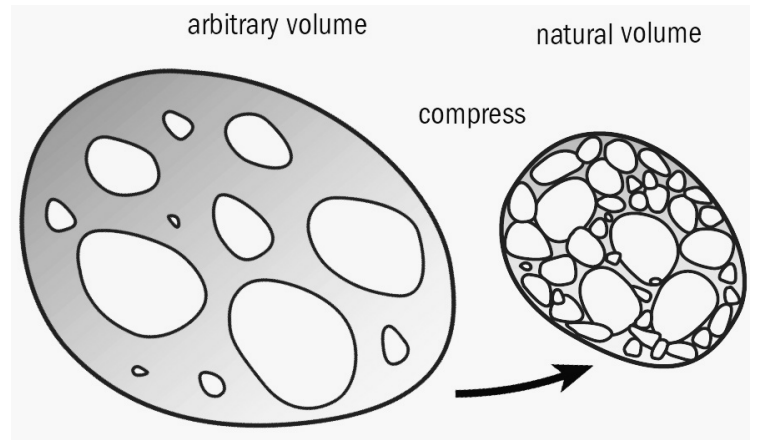

Fig. 3. Illustration of the Statistical Bootstrap Model idea: a volume comprising a gas of fireballs when compressed to natural volume is itself again a fireball. Drawing from ref. [20] modified for this review.

is presented as being a consequence of dynamical collision processes both among hadrons and in QGP, and the dominance of gluon-fusion processes in QGP is described. The role of strangeness in QGP search experiments is presented. For a more extensive historical recount see ref. [18].

\section{The concepts: Theory hadron side}

\subsection{What is the Statistical Bootstrap Model (SBM)?}

Considering that the interactions between hadronic particles are well characterized by resonant scattering, see subsect. 2.4, we can describe the gas of interacting hadrons as a mix of all possible particles and their resonances " $i$ ". This motivates us to consider the case of a gas comprising several types of particles of mass $m_{i}$, enclosed in a heat bath at temperature $T$, where the individual populations " $i$ " are unconstrained in their number, that is like photons in a black box adapting abundance to what is required for the ambient $T$. The non-relativistic limit of the partition function this gas takes the form

$$
\ln Z=\sum_{i} \ln Z_{i}=V\left(\frac{T}{2 \pi}\right)^{3 / 2} \sum_{i} m_{i}^{3 / 2} \mathrm{e}^{-m_{i} / T},
$$

where the momentum integral was carried out and the sum " $i$ " includes all particles of differing parity, spin, isospin, baryon number, strangeness etc. Since each state is counted, there is no degeneracy factor.

It is convenient to introduce the mass spectrum $\rho(m)$, where

$\rho(m) \mathrm{d} m=$ number of " $i$ " hadron states in $\{m, m+\mathrm{d} m\}$

Thus we have

$$
Z(T, V)=\exp \left[V\left(\frac{T}{2 \pi}\right)^{3 / 2} \int_{0}^{\infty} \rho(m) m^{3 / 2} \mathrm{e}^{-m / T} \mathrm{~d} m\right] .
$$

On the other hand, a hadronic fireball comprising many components seen on the left in fig. 3, when compressed to its natural volume $V \rightarrow V_{0}$, is itself a highly excited hadron, a resonance that we must include in eq. (3). This is what Hagedorn realized in 1964 [11]. This observation leads to an integral equation for $\rho(m)$ when we close the "bootstrap" loop that emerges.

Frautschi [12] transcribed Hagedorn's grand canonical formulation into microcanonical format. The microcanonical bootstrap equation reads in invariant Yellin [19] notation

$$
\begin{aligned}
H \tau\left(p^{2}\right)= & H \sum_{m_{\mathrm{in}}} \delta_{0}\left(p^{2}-m_{\mathrm{in}}^{2}\right) \\
& +\sum_{n=2}^{\infty} \frac{1}{n !} \int \delta^{4}\left(p-\sum_{i=1}^{n} p_{i}\right) \prod_{i=1}^{n} H \tau\left(p_{i}^{2}\right) \mathrm{d}^{4} p_{i}
\end{aligned}
$$

where $H$ is a universal constant assuring that eq. (4) is dimensionless; $\tau\left(p^{2}\right)$ on the left-hand side of eq. (4) is the fireball mass spectrum with the mass $m=\sqrt{p_{\mu} p^{\mu}}$ which we are seeking to model. The right-hand side of eq. (4) expresses that the fireball is either just one input particle of a given mass $m_{\mathrm{in}}$, or else composed of several (two or more) particles $i$ having mass spectra $\tau\left(p_{i}^{2}\right)$, and

$$
\tau\left(m^{2}\right) \mathrm{d} m^{2} \equiv \rho(m) \mathrm{d} m .
$$

A solution to eq. (4) has naturally an exponential form

$$
\rho(m) \propto m^{-a} \exp \left(m / T_{\mathrm{H}}\right) .
$$

The appearance of the exponentially growing mass spectrum, eq. (6), is a key SBM result. One of the important consequences is that the number of different hadron states grows so rapidly that practically every strongly interacting particle found in the fireball is distinguishable. Hagedorn realized that the distinguishability of hadron states was an essential input in order to reconcile statistical hadron multiplicities with experimental data. Despite his own initial rejection of a draft paper, see chapts. 18 and 19 loc. cit., this insight was the birth of the theory of hot hadronic matter as it produced the next step, a model [13].

SBM solutions provide a wealth of information including the magnitude of the power index $a$ seen in eq. (6). Frautschi, Hamer, Carlitz [12,21-25] studied solutions to eq. (4) analytically and numerically and by 1975 drew important conclusions:

- Fireballs would predominantly decay into two fragments, one heavy and one light.

- By iterating their bootstrap equation with realistic input, they found numerically $T_{\mathrm{H}} \approx 140 \mathrm{MeV}$ and $a=2.9 \pm 0.1$, which ruled out the previously adopted approximate value $[11,26] a=5 / 2$.

- Each imposed conservation law implemented by fixing a quantum number, e.g., baryon number $\rho(B, m)$, in the mass spectrum, increases the value of $a$ by $1 / 2$.

Werner Nahm independently obtained $a=3$ [27]. Further refinement was possible. In ref. [15], a SBM with compressible finite-size hadrons is introduced where one must 
Table 1. Thermodynamic quantities assuming exponential form of hadron mass spectrum with pre-exponential index $a$, eq. (6); results from ref. [28].

\begin{tabular}{|l||c|c|c|}
\hline$a$ & $P$ & $\varepsilon$ & $\delta \varepsilon / \varepsilon$ \\
\hline \hline $1 / 2$ & $C / \Delta T^{2}$ & $C / \Delta T^{3}$ & $C+C \Delta T$ \\
\hline 1 & $C / \Delta T^{3 / 2}$ & $C / \Delta T^{5 / 2}$ & $C+C \Delta T^{3 / 4}$ \\
\hline $3 / 2$ & $C / \Delta T$ & $C / \Delta T^{2}$ & $C+C \Delta T^{1 / 2}$ \\
\hline 2 & $C / \Delta T^{1 / 2}$ & $C / \Delta T^{3 / 2}$ & $C+C \Delta T^{1 / 4}$ \\
\hline $5 / 2$ & $C \ln \left(T_{0} / \Delta T\right)$ & $C / \Delta T$ & $C$ \\
\hline 3 & $P_{0}-C \Delta T^{1 / 2}$ & $C / \Delta T^{1 / 2}$ & $C / \Delta T^{1 / 4}$ \\
\hline $7 / 2$ & $P_{0}-C \Delta T$ & $\varepsilon_{0}$ & $C / \Delta T^{1 / 2}$ \\
\hline 4 & $P_{0}-C \Delta T^{3 / 2}$ & $\varepsilon_{0}-C \Delta T^{1 / 2}$ & $C / \Delta T^{3 / 4}$ \\
\hline
\end{tabular}

consistently replace eq. (29) by eq. (30). This leads to a finite energy density already for a model which produces $a=3$ with incompressible hadrons.

For any $\rho(m)$ with a given value of $a$, eq. (6), it is easy to understand the behavior near to $T_{\mathrm{H}}$. Inserting eq. (6) into the relativistic form of eq. (1), see chapt. 23 loc. cit. allows the evaluation near critical condition, $T_{\mathrm{H}}-T \equiv$ $\Delta T \rightarrow 0$ of the physical properties such as shown in table 1: pressure $P$, energy density $\varepsilon$, and other physical properties, as example the mean relative fluctuations $\delta \varepsilon / \varepsilon$ of $\varepsilon$ are shown, for $a=1 / 2,2 / 2, \ldots, 8 / 2$. We see that, as $T \rightarrow T_{\mathrm{H}}(\Delta T \rightarrow 0)$, the energy density diverges for $a \leq 3$.

In view of the entries shown in table 1 an important further result can be obtained using these leading order terms for all cases of $a$ considered: the speed of sound at which the small density perturbations propagate

$$
c_{s}^{2}:=\frac{\mathrm{d} P}{\mathrm{~d} \varepsilon} \propto \Delta T \rightarrow 0
$$

This universal for all $a$ result is due to the exponential mass spectrum of hadron matter studied here. $c_{s}^{2} \rightarrow 0$ at $T_{\mathrm{H}}$ harbors an interesting new definition of the phase boundary in the context of lattice-QCD. A non-zero but small value $c_{s}^{2}$ should arise from the subleading terms contributing to $P$ and $\varepsilon$ not shown in table 1 . The way singular properties work, it could be that the $c_{s}^{2}=0$ point exists. The insight that the sound velocity vanishes at $T_{\mathrm{H}}$ is known since 1978, see ref. [28]. An "almost" rediscovery of this result is seen in sects. 3.5 and 8.7 of ref. [29].

The above discussion shows both the ideas that led to the invention of SBM, and how SBM can evolve with our understanding of the strongly interacting matter, becoming more adapted to the physical properties of the elementary "input" particles. Further potential refinements include introducing strange quark related scale into characterization of the hadron volume, making baryons more compressible as compared to mesons. These improvements could generate a highly realistic shape of the mass spectrum, connecting SBM more closely to the numerical study of QCD in lattice approach. We will return to SBM, and the mass spectrum, and describe the method of finding a solution of eq. (4) in sect. 8.
Table 2. Parameters of eq. (8) fitted for a prescribed preexponential power $a$. Results from ref. [30].

\begin{tabular}{|l||c|c|c|c|}
\hline \multicolumn{1}{|c||}{$a$} & $c\left[\mathrm{GeV}^{a-1}\right]$ & $m_{0}[\mathrm{GeV}]$ & $T_{\mathrm{H}}[\mathrm{MeV}]$ & $T_{\mathrm{H}}\left[10^{12} \cdot \mathrm{K}\right]$ \\
\hline \hline 2.5 & 0.83479 & 0.6346 & 165.36 & 1.9189 \\
3. & 0.69885 & 0.66068 & 157.60 & 1.8289 \\
3.5 & 0.58627 & 0.68006 & 150.55 & 1.7471 \\
4. & 0.49266 & 0.69512 & 144.11 & 1.6723 \\
5. & 0.34968 & 0.71738 & 132.79 & 1.5410 \\
6. & 0.24601 & 0.73668 & 123.41 & 1.4321 \\
\hline
\end{tabular}

\subsection{What is the Hagedorn temperature $T_{H}$ ?}

Hagedorn temperature is the parameter entering the exponential mass spectrum eq. (6). It is measured by fitting to data the exponential shape of the hadron mass spectrum. The experimental mass spectrum is discrete; hence a smoothing procedure is often adopted to fit the shape eq. (8) to data. In technical detail one usually follows the method of Hagedorn (see chapt. 20 loc. cit. and ref. [26]), applying a Gaussian distribution with a width of $200 \mathrm{MeV}$ for all hadron mass states. However, the accessible experimental distribution allows fixing $T_{\mathrm{H}}$ uniquely only if we know the value of the pre-exponential power " $a$ ".

The fit procedure is encumbered by the singularity for $m \rightarrow 0$. Hagedorn proposed a regularized form of eq. (6)

$$
\rho(m)=c \frac{e^{m / T_{\mathrm{H}}}}{\left(m_{0}^{2}+m^{2}\right)^{a / 2}} .
$$

In fits to experimental data all three parameters $T_{\mathrm{H}}, m_{0}, c$ must be varied and allowed to find their best value. In 1967 Hagedorn fixed $m_{0}=0.5 \mathrm{GeV}$ as he was working in the limit $m>m_{0}$, and he also fixed $a=2.5$ appropriate for his initial SBM approach [26]. The introduction of a fitted value $m_{0}$ is necessary to improve the characterization of the hadron mass spectrum for low values of $m$, especially when a range of possible values for $a$ is considered.

The fits to experimental mass spectrum shown in table 2 are from 1994 [30] and thus include a smaller set of hadron states than is available today. However, these results are stable since the new hadronic states found are at high mass. We see in table 2 that as the pre-exponential power law $a$ increases, the fitted value of $T_{\mathrm{H}}$ decreases. The value of $c$ for $a=2.5$ corresponds to $c=2.64 \times 10^{4} \mathrm{MeV}^{3 / 2}$, in excellent agreement to the value obtained by Hagedorn in 1967 . In fig. 4 the case $a=3$ is illustrated and compared to the result of the 1967 fit by Hagedorn and the experimental smoothed spectrum. All fits for different $a$ were found at nearly equal and convincing confidence level as can be inferred from fig. 4 .

Even cursory inspection of table 2 suggests that the value of $T_{\mathrm{H}}$ that plays an important role in physics of RHI collisions depends on the understanding of the value of $a$. This is the reason that we discussed the different cases in depth in previous subsect. 2.1. The pre-exponential power value $a=2.5$ in eq. (8) corresponds to Hagedorn's original 


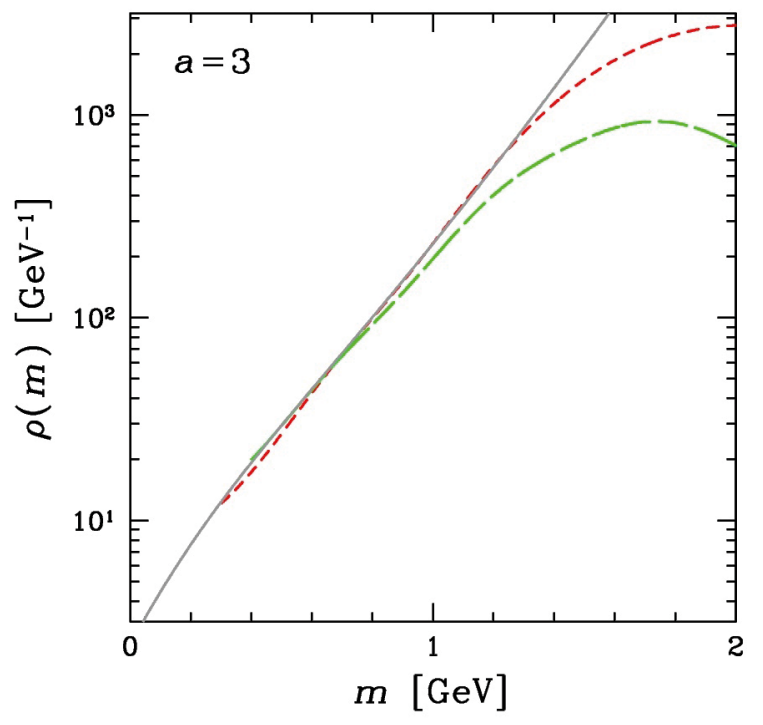

Fig. 4. The experimental mass spectrum (solid line), the fit (short dashed), compared to 1967 fit of Hagedorn (long dashed): The case $a=3$ is shown, for parameters see table 2 . Figure from ref. [20] with results obtained in [31] modified for this review.

preferred value; the value $a=3$ was adopted by the mid70s following extensive study of the SBM as described. However, results seen in table 1 and ref. [15] imply $a \geq$ $7 / 2$.

This is so since for $a<7 / 2$ we expect $T_{\mathrm{H}}$ to be a maximum temperature, for which we see in table 1 a divergence in energy density. Based on study of the statistical bootstrap model of nuclear matter with conserved baryon number and compressible hadrons presented in ref. [15], I believe that $3.5 \leq a \leq 4$. A yet greater value $a \geq 4$ should emerge if in addition strangeness and charge are introduced as a distinct conserved degree of freedom - in any consistently formulated SBM with canonically conserved quantum numbers one unique value of $T_{\mathrm{H}}$ will emerge for the mass spectrum, that is $\rho(m, b, S, \ldots) \propto \exp \left(m / T_{\mathrm{H}}\right)$ for any value of $b, S, Q, \ldots$, i.e. the same $T_{\mathrm{H}}$ for mesons and baryons. Only the pre-exponential function can depend on $b, S, Q, \ldots$ An example for this is provided by the SBM model of Beitel, Gallmeister and Greiner [32]. Using a conserved discrete quantum numbers approach, explicit fits lead to the same (within $1 \mathrm{MeV}$ ) value of $T_{\mathrm{H}}$ for mesons and baryons [32].

These results of ref. [32] are seen in fig. 5: the top frame for mesons and the bottom frame for baryons. Two different fits are shown characterized by a model parameter $R$ which, though different from $H$ seen in eq. (15) in ref. [15], plays a similar role. Thus the two results bracket the value of $T_{\mathrm{H}}$ from above (blue, $T_{\mathrm{H}} \simeq 162 \mathrm{MeV}$ ) and from below (red, $\left.T_{\mathrm{H}} \simeq 145 \mathrm{MeV}\right)$ in agreement with typical empirical results seen in table 2 .

We further see in fig. 5 that a noticeably different number of $M>2 \mathrm{GeV}$ states can be expected depending on the value of $T_{\mathrm{H}}$, even if the resonances for $M<1.7 \mathrm{GeV}$ are equally well fitted in both cases. Thus it would seem

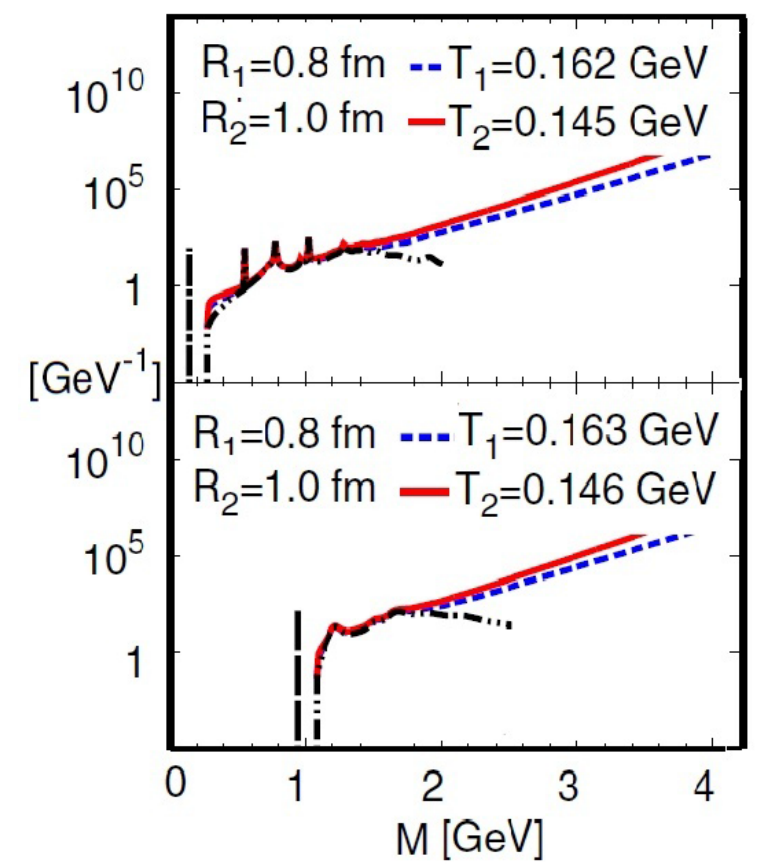

Fig. 5. Meson- (top) and baryon- (bottom) mass spectra $\rho(M)$ (particles per $\mathrm{GeV}$ ): dashed line the experimental spectrum including discrete states. Two different fits are shown, see test. Figure from ref. [32] modified for this review.

that the value of $T_{\mathrm{H}}$ can be fixed more precisely in the future when more hadronic resonances are known. However, for $M \simeq 3 \mathrm{GeV}$ there are about $10^{5}$ different meson or baryon states per GeV. This means that states of this mass are on average separated by $10 \mathrm{eV}$ in energy. On the other hand, their natural width is at least $10^{6}$ larger. Thus there is little if any hope to experimentally resolve such "Hagedorn" states. Hence we cannot expect to determine, based on experimental mass spectrum, the value of $T_{\mathrm{H}}$ more precisely than it is already done today. However, there are other approaches to measure the value of $T_{\mathrm{H}}$. For example, we address at the end of subsect. 3.3 why the behavior of lattice-QCD determined speed of sound suggests that $T_{\mathrm{H}} \simeq 145 \mathrm{MeV}$.

To summarize, our current understanding is that Hagedorn temperature has a value still needing an improved determination,

$$
140 \leq T_{\mathrm{H}} \leq 155 \mathrm{MeV} \quad T_{\mathrm{H}} \simeq(1.7 \pm 0.1) \times 10^{12} \mathrm{~K} .
$$

$T_{\mathrm{H}}$ is the maximum temperature at which matter can exist in its usual form. $T_{\mathrm{H}}$ is not a maximum temperature in the Universe. The value of $T_{\mathrm{H}}$ which we evaluate in the study of hadron mass spectra is, as we return to discuss in sect. 3.3, the melting point of hadrons dissolving into the quark-gluon plasma (QGP), a liquid phase made of Debye-screened color-ionic quarks and gluons. A further heating of the quark-gluon plasma "liquid" can and will continue. A similar transformation can occur already at a lower temperature at a finite baryon density.

Indeed, there are two well studied ways to obtain deconfinement: a) high temperature; and b) high baryon 
density. In both cases the trick is that the number of particles per unit volume is increased.

a) In the absence of all matter (zero net baryon number corresponding to baryochemical potential $\mu_{\mathrm{B}} \rightarrow 0$ ), in full thermal equilibrium temperature alone controls the abundance of particles as we already saw in the context of SBM. The result of importance to this review is that confinement is shown to dissolve in the study of QCD by Polyakov [33], and this has been also argued early on and independently in the context of lattice-QCD [34].

b) At nuclear (baryon) densities an order of magnitude greater than the prevailing nuclear density in large nuclei, this transformation probably can occur near to, or even at, zero temperature; for further quantitative discussion see ref. [15]. This is the context in which asymptotically free quark matter was proposed in the context of neutron star physics [35].

Cabibbo and Parisi [36] were first to recognize that these two distinct limits are smoothly connected and that the phase boundary could be a smooth line in the $\mu_{\mathrm{B}}, T$ plane. Their qualitative remarks did not address a method to form, or to explore, the phase boundary connecting these limits. The understanding of high baryon density matter properties in the limit $T \rightarrow 0$ is a separate vibrant research topic which will not be further discussed here [37-40]. Our primary interest is the domain in which the effects of temperature dominate, in this sense the limit of small $\mu_{\mathrm{B}} \ll T$.

\subsection{Are there several possible values of $T_{H}$ ?}

The singularity of the SBM at $T_{\mathrm{H}}$ is a unique singular point of the model. If and when within SBM we implement distinguishability of mesons from baryons, and/or of strange and non-strange hadrons, all these families of particles would have a mass spectrum with a common value of $T_{\mathrm{H}}$. No matter how complex are the so-called SBM "input" states, upon Laplace transform they always lead to one singular point, see subsect. 8.3. In subsequent projection of the generating SBM function onto individual families of hadrons one common exponential is found for all. On the other hand, it is evident from the formalism that when extracting from the common expression the specific forms of the mass spectrum for different particle families, the preexponential function must vary from family to family. In concrete terms this means that we must fit the individual mass spectra with common $T_{\mathrm{H}}$ but particle family dependent values of $a$ and dimensioned parameter $c, m_{0}$ seen in table 2, or any other assumed pre-exponential function.

There are several recent phenomenological studies of the hadron mass spectrum claiming to relate to SBM of Hagedorn, and the approaches taken are often disappointing. The frequently seen defects are: i) Assumption of $a=2.5$ along with the Hagedorn 1964-67 model, a value obsolete since 1971 when $a=3$ and higher was recognized; and ii) Choosing to change $T_{\mathrm{H}}$ for different particle families, e.g. baryons and mesons or strange/non-strange hadrons instead of modifying the pre-exponential function for different particle families. iii) A third technical problem is that an integrated ("accumulated") mass spectrum is considered,

$$
R(M)=\int_{0}^{M} \rho(m) \mathrm{d} m
$$

While the Hagedorn-type approach requires smoothing of the spectrum, adopting an effective Gaussian width for all hadrons, the integrated spectrum eq. (10) allows one to address directly the step function arising from integrating the discrete hadron mass spectrum, i.e. avoiding the Hagedorn smoothing. One could think that the Hagedorn smoothing process loses information that is now available in the new approach, eq. (10). However, it also could be that a greater information loss comes from the consideration of the integrated "signal". This situation is not uncommon when considering any integrated signal function.

The Krakow group refs. [41,42] was first to consider the integrated mass spectrum eq. (10). They also break the large set of hadron resonances into different classes, e.g. non-strange/strange hadrons, or mesons/baryons. However, they chose same pre-exponential fit function and varied $T_{\mathrm{H}}$ between particle families. The fitted value of $T_{\mathrm{H}}$ was found to be strongly varying in dependence on supplementary hypotheses made about the procedure, with the value of $T_{\mathrm{H}}$ changing by 100 's $\mathrm{MeV}$, possibly showing the inconsistency of procedure aggravated by the loss of signal information.

Reference [43] fixes $m_{0}=0.5 \mathrm{GeV}$ at $a=2.5$, i.e. Hagedorn's 1968 parameter choices. Applying the Krakow method approach, this fit produces with present day data $T_{\mathrm{H}}=174 \mathrm{MeV}$. We keep in mind that the assumed value of $a$ is incompatible with SBM, while the assumption of a relatively small $m_{0}=0.5 \mathrm{GeV}$ is forcing a relatively large value of $T_{\mathrm{H}}$, compare here also the dependence of $T_{\mathrm{H}}$ on $a$ seen in table 2. Another similar work is ref. [44], which seeing poor phenomenological results that emerge from an inconsistent application of Hagedorn SBM, criticizes unjustly the current widely accepted Hagedorn approach and Hagedorn temperature. For reasons already described, we do not share in any of the views presented in this work.

However, we note two studies $[45,46]$ of differentiated (meson vs. baryon) hadron mass spectrum done in the way that we consider correct: using a common singularity, that is one and the same exponential $T_{\mathrm{H}}$, but "family" dependent pre-exponential functions obtained in projection on the appropriate quantum number. It should be noted that the hadronic volume $V_{h}$ enters any reduction of the mass spectrum by the projection method, see ref. [15], where volume effect for strangeness is shown.

Biro and Peshier [45] search for $T_{\mathrm{H}}$ within nonextensive thermodynamics. They consider two different values of $a$ for mesons and baryons (somewhat on the low side), and in their fig. 2 the two fits show a common value of $T_{\mathrm{H}}$ around $150-170 \mathrm{MeV}$. A very recent lattice motivated effort assumes differing shape of the pre-exponential function for different families of particles [46], and uses a common, but assumed, not fitted, value of $T_{\mathrm{H}}$. 
Arguably, the most important recent step forward in regard to improving the Hagedorn mass spectrum analysis is the realization first made by Majumder and Müller [47] that one can infer important information about the hadron mass spectrum from lattice-QCD numerical results. However, this first effort also assumed $a=2.5$ without a good reason. Moreover, use of asymptotic expansions of the Bessel functions introduced errors, preventing a comparison of these results with those seen in table 2 .

To close let us emphasize that phenomenological approach in which one forces same pre-exponential function and fits different values of $T_{\mathrm{H}}$ for different families of particles is at least within the SBM framework blatantly wrong. A more general argument indicating that this is always wrong could be also made: the only universal natural constant governing phase boundary is the value of $T_{\mathrm{H}}$, the preexponential function, which varies depending on how we split up the hadron particle family - projection of baryon number (meson, baryon), and strangeness, are two natural choices.

\subsection{What is hadron resonance gas (HRG)?}

We are seeking a description of the phase of matter made of individual hadrons. One would be tempted to think that the SBM provides a valid framework. However, we already know from discussion above that the experimental realities limit the ability to fix the parameters of this model; specifically, we do not know $T_{\mathrm{H}}$ precisely.

In the present day laboratory experiments one therefore approaches the situation differently. We employ all experimentally known hadrons as explicit partial fractions in the hadronic gas: this is what in general is called the hadron resonance gas (HRG), a gas represented by the non-averaged, discrete sum partial contributions, corresponding to the discrete format of $\rho(m)$ as known empirically.

The emphasis here is on "resonances" gas, reminding us that all hadrons, stable and unstable, must be included. In his writings Hagedorn went to great length to justify how the inclusion of unstable hadrons, i.e. resonances, accounts for the dominant part of the interaction between all hadronic particles. His argument was based on work of Belenky (also spelled Belenkij) [48], but the intuitive content is simple: if and when reaction cross sections are dominated by resonant scattering, we can view resonances as being all the time present along with the scattering particles in order to characterize the state of the physical system. This idea works well for strong interactions since the S-matrix of all reactions is pushed to its unitarity limit.

To illustrate the situation, let us imagine a hadron system at "low" $T \simeq T_{\mathrm{H}} / 5$ and at zero baryon density; this is in essence a gas made of the three types of pions, $\pi^{(+,-, 0)}$. In order to account for dominant interactions between pions we include their scattering resonances as individual contributing fractions. Given that these particles have considerably higher mass compared to that of two pions, their number is relatively small.

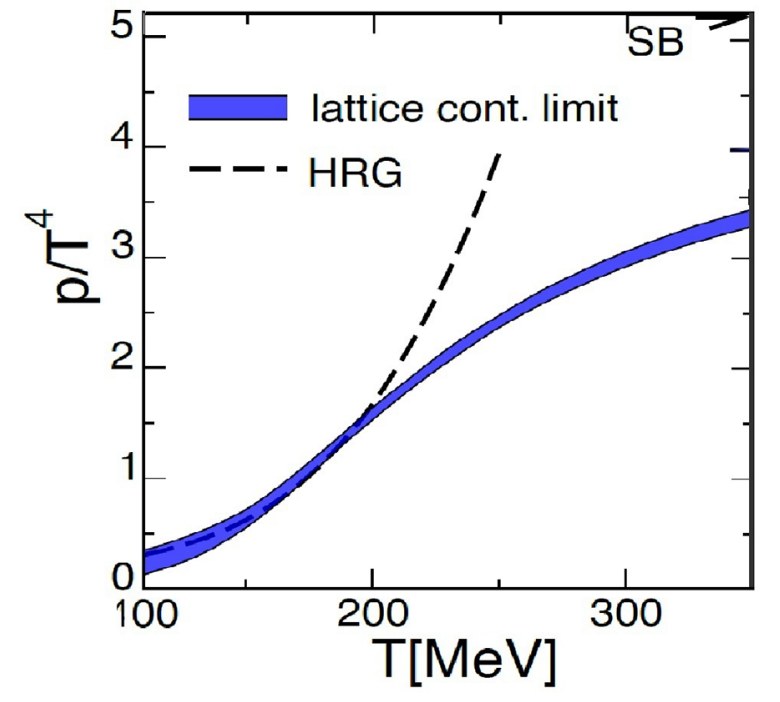

Fig. 6. Pressure $P / T^{4}$ of QCD matter evaluated in lattice approach (includes $2+1$ flavors and gluons) compared with their result for the HRG pressure, as function of $T$. The upper limit of $P / T^{4}$ is the free Stephan-Boltzmann (SB) quark-gluon pressure with three flavors of quarks in the relativistic limit $T \gg$ strange quark mass. Figure from ref. [72] modified for this review.

But as we warm up our hadron gas, for $T>T_{\mathrm{H}} / 5$ resonance contribution becomes more noticeable and in turn their scattering with pions requires inclusion of other resonances and so on. As we reach $T \lesssim T_{\mathrm{H}}$ in the heat-up process, Hagedorn's distinguishable particle limit applies: very many different resonances are present such that this hot gas develops properties of classical numbered-ball system, see chapt. 19 loc. cit.

All heavy resonances ultimately decay, the process creating pions observed experimentally. This yield is well ahead of what one would expect from a pure pion gas. Moreover, spectra of particles born in resonance decays differ from what one could expect without resonances. As a witness of the early Hagedorn work from before 1964, Maria Fidecaro of CERN told me recently, I paraphrase "when Hagedorn produced his first pion yields, there were many too few, and with a wrong momentum spectrum". As we know, Hagedorn did not let himself be discouraged by this initial difficulty.

The introduction of HRG can be tested theoretically by comparing HRG properties with lattice-QCD. In fig. 6 we show the pressure presented in ref. [72]. We indeed see a good agreement of lattice-QCD results obtained for $T \lesssim T_{\mathrm{H}}$ with HRG, within the lattice-QCD uncertainties. In this way we have $a b$ initio confirmation that Hagedorn's ideas of using particles and their resonances to describe a strongly interacting hadron gas is correct, confirmed by more fundamental theoretical ideas involving quarks, gluons, QCD.

Results seen in fig. 6 comparing pressure of latticeQCD with HRG show that, as temperature decreases towards and below $T_{\mathrm{H}}$, the color charge of quarks and gluons literally freezes, and for $T \lesssim T_{\mathrm{H}}$ the properties of 
strongly interacting matter should be fully characterized by a HRG. Quoting Redlich and Satz [49]:

"The crucial question thus is, if the equation of state of hadronic matter introduced by Hagedorn can describe the corresponding results obtained from QCD within lattice approach." and they continue: "There is a clear coincidence of the Hagedorn resonance model results and the lattice data on the equation of states. All bulk thermodynamical observables are very strongly changing with temperature when approaching the deconfinement transition. This behavior is well understood in the Hagedorn model as being due to the contribution of resonances. ... resonances are indeed the essential degrees of freedom near deconfinement. Thus, on the thermodynamical level, modeling hadronic interactions by formation and excitation of resonances, as introduced by Hagedorn, is an excellent approximation of strong interactions."

\subsection{What does lattice-QCD tell us about HRG and about the emergence of equilibrium?}

The thermal pressure reported in fig. 6 is the quantity least sensitive to missing high mass resonances which are nonrelativistic and thus contribute little to pressure. Thus the agreement we see in fig. 6 is testing: a) the principles of Hagedorn's HRG ideas; and b) consistency with the part of the hadron mass spectrum already known, see fig. 4. A more thorough study is presented in subsect. 8.5, describing the compensating effect for pressure of finite hadron size and missing high mass states in HRG, which than produces good fit to energy density.

Lattice-QCD results apply to a fully thermally equilibrated system filling all space-time. This in principle is true only in the early Universe. After hadrons are born at $T \lesssim T_{\mathrm{H}}$, the Universe cools in expansion and evolves, with the expansion time constant governed by the magnitude of the (applicable to this period) Hubble parameter; one finds $[6,50] \tau_{q} \propto 25 \cdot \mu \mathrm{s}$ at $T_{\mathrm{H}}$, see also subsect. 7.4. The value of $\tau_{q}$ is long on hadron scale. A full thermal equilibration of all HRG particle components can be expected in the early Universe.

Considering the early Universe conditions, it is possible and indeed necessary to interpret the lattice-QCD results in terms of a coexistence era of hadrons and QGP. This picture is usually associated with a 1st order phase transition, see Kapusta and Csernai [51] where one finds separate spatial domains of quarks and hadrons. However, as one can see modeling the more experimentally accessible smooth transition of hydrogen gas to hydrogen plasma, this type of consideration applies in analogy also to any smooth phase transformation. The difference is that for smooth transformation, the coexistence means that the mixing of the two phases is complete at microscopic level; no domain formation occurs. However, the physical properties of the mixed system like in the 1st order transition case are obtained in a superposition of fractional gas components.

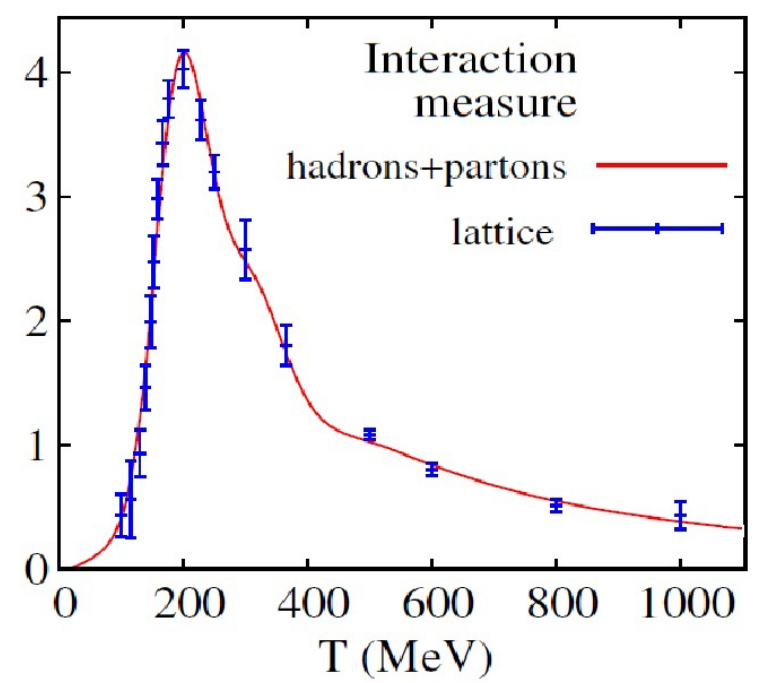

Fig. 7. The Interaction measure $(\varepsilon-3 P) / T^{4}$ within mixed parton-hadron model, model fitted to match the lattice data of ref. [72]. Figure from ref. [52] modified for this review.

The recent analysis of lattice-QCD results of Biro and Jakovac [52] proceeds in terms of a perfect microscopic mix of partons and hadrons. One should take note that as soon as QCD-partons appear, in such a picture color deconfinement is present. In figs. 10 and 11 in [52] the appearance of partons for $T>140 \mathrm{MeV}$ is noted. Moreover, this model is able to describe precisely the interaction measure

$$
I_{\mathrm{m}} \equiv \frac{\varepsilon-3 P}{T^{4}}
$$

as shown in fig. 7. $I_{\mathrm{m}}$ is a dimensionless quantity that depends on the scale invariance violation in QCD. We note the maximum value of $I_{\mathrm{m}} \simeq 4.2$ in fig. 7 , a value which reappears in the hadronization fit in fig. 35, subsect. 10.2, where we see for a few classes of collisions the same value $I_{\mathrm{m}} \simeq 4.6 \pm 0.2$.

Is this agreement between a hadronization fit and lattice $I_{\mathrm{m}}$ an accident? The question is open since a priori this agreement has to be considered allowing for the rapid dynamical evolution occurring in laboratory experiments, a situation differing vastly from the lattice simulation of static properties. The dynamical situation is also more complex and one cannot expect that the matter content of the fireball is a parton-hadron ideal mix. The rapid expansion could and should mean that the parton system evolves without having time to enter equilibrium mixing with hadrons, this is normally called super-cooling in the context of a 1st order phase transition, but in context of a mix of partons and hadrons [52], these ideas should also apply: as the parton phase evolves to lower temperature, the yet non-existent hadrons will need to form.

To be specific, consider a dense hadron phase created in RHI collisions with a size $R_{h} \propto 5 \mathrm{fm}$ and a $T \simeq 400 \mathrm{MeV}$, where the fit of ref. [52] suggest small if any presence of hadrons. Exploding into space this parton domain dilutes at, or even above, the speed of sound in the transverse direction and even faster into the longitudinal 


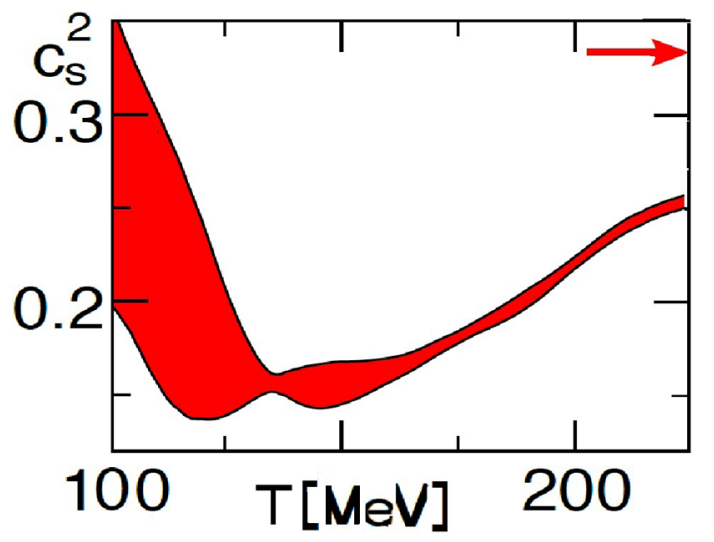

Fig. 8. The square of speed of sound $c_{s}^{2}$ as function of temperature $T$, the relativistic limit is indicated by an arrow. Figure from ref. [72] modified for this review.

direction. For relativistic matter the speed of sound eq. (7) approaches $c_{s}=c / \sqrt{3}$, see fig. 8 and becomes small only near to $T_{\mathrm{H}}$. Within time $\tau_{h} \propto 10^{-22} \mathrm{~s}$ a volume dilution by a factor 50 and more can be expected.

It is likely that this expansion is too fast to allow hadron population to develop from the parton domain. What this means is that for both the lattice-QGP interpreted as parton-hadron mix, and for a HRG formed in laboratory, the reaction time is too short to allow development of a multi-structure hadron abundance equilibrated state, which one refers to as "chemical" equilibrated hadron gas, see here the early studies in refs. [53$55]$.

To conclude: lattice results allow various interpretations, and HRG is a consistent simple approximation for $T \lesssim 145 \mathrm{MeV}$. More complex models which include coexistence of partons and hadrons manage a good fit to all lattice results, including the hard to get interaction measure $I_{\mathrm{m}}$. Such models in turn can be used in developing dynamical model of the QGP fireball explosion. One can argue that the laboratory QGP cannot be close to the full chemical equilibrium; a kinetic computation will be needed to assess how the properties of parton-hadron phase evolve given a characteristic lifespan of about $\tau_{h} \propto 10^{-22} \mathrm{~s}$. Such a study may be capable of justifying accurately specific hadronization models.

\subsection{What does lattice-QCD tell us about $T_{H}$ ?}

We will see in subsect. 3.3 that we do have two different lattice results showing identical behavior at $T \in$ $\{150 \pm 25\} \mathrm{MeV}$. This suggests that it should be possible to obtain a narrow range of $T_{\mathrm{H}}$. Looking at fig. 6 , some see $T_{\mathrm{H}}$ at $140-145 \mathrm{MeV}$, others as high as $170 \mathrm{MeV}$. Such disparity can arise when using eyesight to evaluate fig. 6 without applying a valid criterion. In fact such a criterion is available if we believe in exponential mass spectrum.

When presenting critical properties of SBM table 1 we reported that sound velocity eq. (7) has the unique property $c_{s} \rightarrow 0$ for $T \rightarrow T_{\mathrm{H}}$. What governs this result is solely the exponential mass spectrum, and this result holds in leading order irrespective of the value of the power index $a$. Thus a surprisingly simple SBM-related criterion for the value of $T_{\mathrm{H}}$ is that there $c_{s} \rightarrow 0$. Moreover, $c_{s}$ is available in lattice-QCD computation. Figure 8 shows $c_{s}^{2}$ as function of $T$, adapted from ref. [72]. There is a noticeable domain where $c_{s}$ is relatively small.

In fig. 8 the bands show the computational uncertainty. To understand better the value of $T_{\mathrm{H}}$ we follow the drop of $c_{s}^{2}$ when temperature increases, and when $c_{s}$ begins to increase that is presumably, in the context of lattice-QCD, when the plasma material is mostly made of deconfined and progressively more mobile quarks and gluons. As temperature rises further, we expect to reach the speed of sound limit of ultra relativistic matter $c_{s}^{2} \rightarrow 1 / 3$, indicated in fig. 8 by an arrow. This upper limit, $c_{s}^{2} \leq 1 / 3$ arises according to eq. (7) as long as the constraint $\varepsilon-3 P \rightarrow 0$ from above at high $T$ applies; that is $I_{\mathrm{m}}>0$ and $I_{\mathrm{m}} \rightarrow 0$ at high $T$.

The behavior of the lattice result-bands in fig. 8 suggests hadron dominance below $T=125 \mathrm{MeV}$, and quark dominance above $T=150 \mathrm{MeV}$. This is a decisively more narrow range compared to the wider one seen in the fit in which a mixed parton-hadron phase was used to describe lattice results [52]; see discussion in subsect. 2.5.

The shape of $c_{s}^{2}$ in fig. 8 suggests that $T_{\mathrm{H}}=138 \pm$ $12 \mathrm{MeV}$. There are many ramifications of such a low value, as is discussed in the context of hadronization model in the following subsect. 2.7.

\subsection{What is the statistical hadronization model (SHM)?}

The pivotal point leading on from the last subsection is that in view of fig. 6 we can say that $\mathrm{HRG}$ for $T \lesssim T_{\mathrm{H}} \simeq$ $150 \mathrm{MeV}$ works well at a precision level that rivals the numerical precision of lattice-QCD results. This result justifies the method of data analysis that we call Statistical Hadronization Model (SHM). SHM was invented to characterize how a blob of primordial matter that we call QGP falls apart into individual hadrons. At zero baryon density this "hadronization" process is expected to occur near if not exactly at $T_{\mathrm{H}}$. The SHM relies on the hypothesis that a hot fireball made of building blocks of future hadrons populates all available phase space cells proportional to their respective size, without regard to any additional interaction strength governing the process.

The model is presented in depth in sect. 9. Here we would like to place emphasis on the fact that the agreement of lattice-QCD results with the HRG provides today a firm theoretical foundation for the use of the SHM, and it sets up the high degree of precision at which SHM can be trusted.

Many argue that Koppe [56,57], and later, independently, Fermi [58] with improvements made by Pomeranchuk [59], invented SHM in its microcanonical format; this is the so called Fermi-model, and that Hagedorn [11, $60]$ used these ideas in computing within grand canonical formulation. However, in all these approaches the particles emitted were not newly formed; they were seen 
as already being the constituents of the fireball. Such models therefore are what we today call freeze-out models.

The difference between QGP hadronization and freezeout models is that a priori we do not know if right at the time of QGP hadronization particles will be born into a condition that allows free-streaming and thus evolve in hadron form to the freeze-out condition. In a freeze-out model all particles that ultimately free-stream to a detector are not emergent from a fireball but are already present. The fact that the freeze-out condition must be established in a study of particle interactions was in the early days of the Koppe-Fermi model of no relevance since the experimental outcome was governed by the phase space and microcanonical constraints as Hagedorn explained in his very vivid account "The long way to the Statistical Bootstrap Model", chapt. 17 loc. cit.

In the Koppe-Fermi-model, as of the instant of their formation, all hadrons are free-streaming. This is also Hagedorn's fireball pot with boiling matter. This reaction view was formed before two different phases of hadronic matter were recognized. With the introduction of a second primordial phase a new picture emerges: there are no hadrons to begin with. In this case in a first step quarks freeze into hadrons at or near $T_{\mathrm{H}}$, and in a second step at $T<T_{\mathrm{H}}$ hadrons decouple into free-streaming particles. It is possible that $T_{\mathrm{H}}$ is low enough so that when the quark freezing into hadrons occurs, hadrons are immediately free-streaming; that is $T \simeq T_{\mathrm{H}}$, in which case one would expect abundances of observed individual particles to be constrained by the properties of QGP, and not of the HRG.

On the other hand if in the QGP hadronization a dense phase of hadron matter should form, this will assure both chemical and thermal equilibrium of later free-streaming hadrons as was clearly explained in 1985 [61]: Why the Hadronic Gas Description of Hadronic Reactions Works: The Example of Strange Hadrons. It is argued that the way parton deconfinement manifests itself is to allow a short lived small dynamical system to reach nearly full thermal and chemical equilibrium.

The analysis of the experimental data within the SHM allows us to determine the degree of equilibration for different collision systems. The situation can be very different in $p p$ and $A A$ collisions and depend on both collision energy and the size $A$ of atomic nuclei, and the related variable describing the variable classes, the participant number $N_{\text {part }}$, see subsect. 9.3. Study of strangeness which is not present in initial RHI states allows us to address the equilibration question in a quantitative way as was noted already 30 years ago [61]. We return to the SHM strangeness results in sect. 10 demonstrating the absence of chemical equilibrium in the final state, and the presence of (near) chemical equilibrium in QGP formed at LHC, see figs. 36 and 38.

One cannot say it strongly enough: the transient presence of the primordial phase of matter means that there are two different possible scenarios describing production of hadrons in RHI collisions: a) A dense fireball disintegrates into hadrons. There can be two temporally separate physical phenomena: the recombinant-evaporative hadronization of the fireball made of quarks and gluons forming a HRG; this is followed by freeze-out; that is, the beginning of free-streaming of the newly created particles.

b) The quark fireball expands significantly before converting into hadrons, reaching a low density before hadronization. As a result, some features of hadrons upon production are already free-streaming: i) The hadronization temperature may be low enough to freeze-out particle abundance (chemical freeze-out at hadronization), yet elastic scattering can still occur and as result momentum distribution will evolve (kinetic non-equilibrium at hadronization). ii) At a yet lower temperature domain, hadrons would be born truly free-streaming and both chemical and kinetic freeze-out conditions would be the same. This condition has been proposed for SPS yields and spectra in the year 2000 by Torrieri [62], and named "single freeze-out" in a later study of RHIC results $[63,64]$.

\subsection{Why value of $\mathrm{T}_{\mathrm{H}}$ matters to $\mathrm{SHM}$ analysis?}

What exactly happens in RHI collisions in regard to particle production depends to a large degree on the value of the chemical freeze-out temperature ${ }^{2} T \leq T_{\mathrm{H}}$. The value of $T_{\mathrm{H}}$ as determined from mass spectrum of hadrons depends on the value of the pre-exponential power index $a$, see table 2 . The lower is $T_{\mathrm{H}}$, the lower the value of $T$ must be. Since the value of $T$ controls the density of particles, as seen in, e.g., eq. (1), the less dense would be the HRG phase that can be formed. Therefore, the lower is $T_{\mathrm{H}}$ the more likely that particles boiled off in the hadronization process emerge without rescattering, at least without the rescattering that changes one type of particle into another i.e. "chemical" free streaming. In such a situation in chemical abundance analysis we expect to find $T \simeq T_{\mathrm{H}}$.

The SHM analysis of particle production allows us to determine both the statistical parameters including the value of $T$ characterizing the hadron phase space, as well as the extensive (e.g., volume) and intensive (e.g., baryon density) physical properties of the fireball source. These govern the outcome of the experiment on the hadron side, and thus can be measured employing experimental data on hadron production as we show in sect. 10 .

The faster is the hadronization process, the more information is retained about the QGP fireball in the hadronic populations we study. For this reason there is a longlasting discussion in regard to how fast or, one often says, sudden is the breakup of QGP into hadrons. Sudden hadronization means that the time between QGP breakup and chemical freeze-out is short as compared to the time needed to change abundances of particles in scattering of hadrons.

\footnotetext{
${ }^{2}$ We omit subscript for all different "temperatures" under consideration - other than $T_{\mathrm{H}}$ - making the meaning clear in the text contents.
} 
Among the source (fireball) observables we note the nearly conserved, in the hadronization process, entropy content, and the strangeness content, counted in terms of the emerging multiplicities of hadronic particles. The physical relevance of these quantities is that they originate, e.g. considering entropy or strangeness yield, at an earlier fireball evolution stage as compared to the hadronization process itself; since entropy can only increase, this provides a simple and transparent example how in hadron abundances which express total entropy content there can be memory of the initial state dynamics.

Physical bulk properties such as the conserved (baryon number), and almost conserved (strangeness pair yields, entropy yield) can be measured independent of how fast the hadronization process is, and independent of the complexity of the evolution during the eventual period in time while the fireball cools from $T_{\mathrm{H}}$ to chemical freeze-out $T$. We do not know how the bulk energy density $\varepsilon$ and pressure $P$ at hadronization after scaling with $T^{4}$ evolve in time to freeze-out point, and even more interesting is how $I_{m}$ eq. (11) evolves. This can be a topic of future study.

Once scattering processes came into discussion, the concept of dynamical models of freeze-out of particles could be addressed. The review of Koch et al. [2] comprises many original research results and includes for the first time the consideration of dynamical QGP fireball evolution into free-streaming hadrons and an implementation of SHM in a format that we could today call SHM with sudden hadronization. In parallel it was recognized that the experimentally observed particle abundances allow the determination of physical properties of the source. This insight is introduced in ref. [16], fig. 3 where we see how the ratio $\mathrm{K}^{+} / \mathrm{K}^{-}$allows the evaluation of the baryochemical potential $\mu_{\mathrm{B}}$; this is stated explicitly in pertinent discussion. Moreover, in the following fig. 4 the comparison is made between abundance of final state $\bar{\Lambda} / \Lambda$ particle ratio emerging from equilibrated HRG with abundance expected in direct evaporation of the quark-fireball an effect that we attribute today to chemical non-equilibrium with enhanced phase space abundance.

Discussion of how sudden the hadronization process is reaches back to the 1986 microscopic model description of strange (antibaryon) formation by Koch, Müller and the author [2] and the application of hadron afterburner. Using these ideas in 1991, SHM model saw its first humble application in the study of strange (anti)baryons [65]. Strange baryon and antibaryon abundances were interpreted assuming a fast hadronization of QGP - fast meaning that their relative yields are little changed in the following evolution. For the past 30 years the comparison of data with the sudden hadronization concept has never led to an inconsistency. Several theoretical studies support the sudden hadronization approach, a sample of works includes refs. [66-70]. Till further notice we must presume that the case has been made.

Over the past 35 years a simple and naive thermal model of particle production has resurfaced multiple times, reminiscent of the work of Hagedorn from the early 60 s. Hadron yields emerge from a fully equilibrated hadron fireball at a given $T, V$ and to account for baryon content at low collision energies one adds $\mu_{\mathrm{B}}$. As Hagedorn found out, the price of simplicity is that the yields can differ from experiment by a factor two or more. His effort to resolve this riddle gave us SBM.

However, in the context of experimental results that need attention, one seeks to understand systematic behavior across yields varying by many orders of magnitude as parameters (collision energy, impact parameter) of RHI collision change. So if a simple model practically "works", for many the case is closed. However, one finds in such a simple model study the value of chemical freezeout $T$ well above $T_{\mathrm{H}}$. This is so since in fitting abundant strange antibaryons there are two possible solutions: either a $T \gg T_{\mathrm{H}}$, or $T \lesssim T_{\mathrm{H}}$ with chemical non-equilibrium. A model with $T \gg T_{\mathrm{H}}$ for the price of getting strange antibaryons right creates other contradictions, one of which is discussed in subsect. 10.4.

How comparison of chemical freeze-out $T$ with $T_{\mathrm{H}}$ works is shown in fig. 9. The bar near to the temperature axis displays the range $T_{\mathrm{H}}=147 \pm 5 \mathrm{MeV}[71,72]$. The symbols show the results of hadronization analysis in the $T-\mu_{\mathrm{B}}$ plane as compiled in ref. [73] for results involving most (as available) central collisions and heaviest nuclei. The solid circles are results obtained using the full SHM parameter set [7,73-79]. The SHARE LHC freeze-out temperature is clearly below the lattice critical temperature range. The results of other groups are obtained with simplified parameter sets: marked GSI [80, 81], Florence [82-84], THERMUS [85], STAR [86] and ALICE $[87,88]$. These results show the chemical freeze-out temperature $T$ in general well above the lattice $T_{\mathrm{H}}$. This means that these restricted SHM studies are incompatible with lattice calculations, since chemical hadron decoupling should not occur inside the QGP domain.

\subsection{How is SHM analysis of data performed?}

Here the procedure steps are described which need technical implementation presented in sect. 9 .

Data: The experiment provides, within a well defined collision class, see subsect. 9.3, spectral yields of many particles. For the SHM analysis we focus on integrated spectra, the particle number-yields. The reason that such data are chosen for study is that particle yields are independent of local matter velocity in the fireball which imposes spectra deformation akin to the Doppler shift. However, if the $p_{\perp}$ coverage is not full, an extrapolation of spectra needs to be made that introduces the same uncertainty into the study. Therefore it is important to achieve experimentally as large as possible $p_{\perp}$ coverage in order to minimize extrapolation errors on particles yields considered.

Evaluation: In first step we evaluate, given an assumed SHM parameter set, the phase space size for all and every particle fraction that could be in principle measured, including resonances. This complete set is necessary since the observed particle set includes particles arising from 


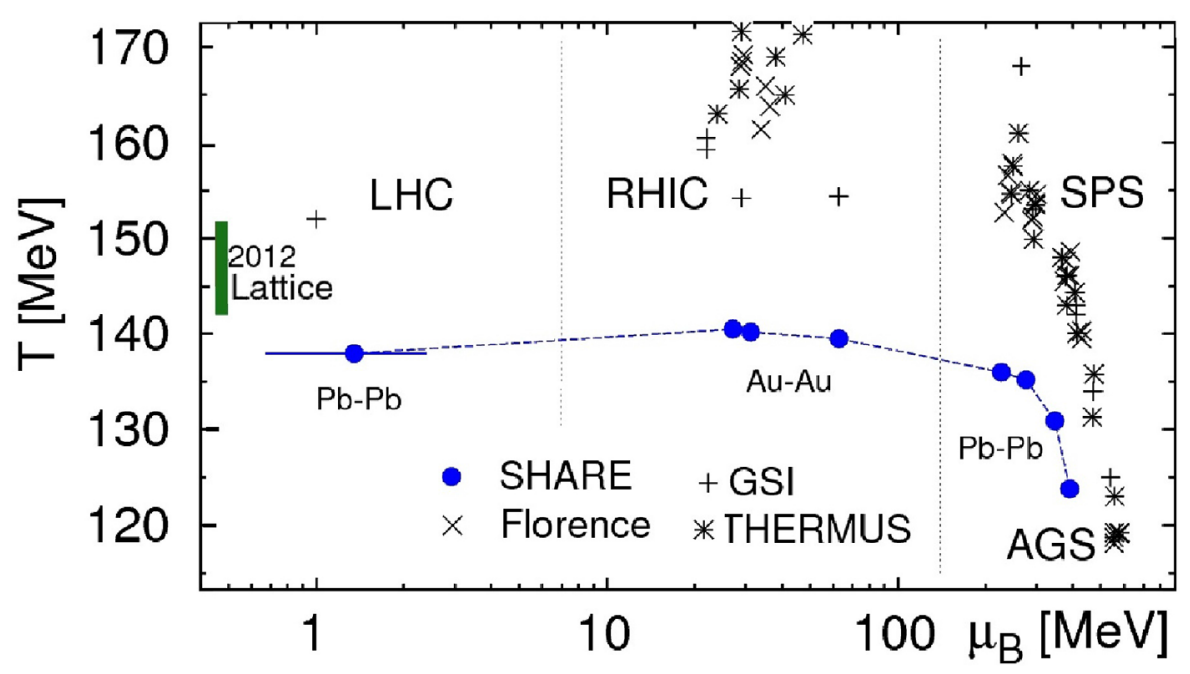

Fig. 9. $T, \mu_{\mathrm{B}}$ diagram showing current lattice value of critical temperature $T_{c}$ (bar on left) [71,72], the SHM-SHARE results (full circles) [7,73-79] and results of other groups [80-88]. Figure from ref. [73] modified for this review.

a sequel chain of resonance decays. These decays are implemented and we obtain the relative phase space size of all potential particle yields.

Optional: Especially should hadronization $T$ be at a relatively large value, the primary particle populations can undergo modifications in subsequent scattering. However, since $T<T_{\mathrm{H}}$, a large $T$ requires an even larger $T_{\mathrm{H}}$ which shows importance of knowing $T_{\mathrm{H}}$. If $T$ is large, a further evolution of hadrons can be treated with hadron "afterburners" taking the system from $T_{\mathrm{H}}$ to $T$. Since in our analysis the value of hadronization $T$ is small, we do not address this stage further here; see however refs. [89,90].

Iteration: The particle yields obtained from phase space evaluation represent the SHM parameter set assumed. A comparison of this predicted yield with observed yields allows the formation of a value parameter such as

$$
\chi^{2}=\sum_{i}(\text { theory-data })^{2} / \mathrm{FWHM}^{2},
$$

where FWHM is the error in the data, evaluated as "Full Width at Half Maximum" of the data set. In an iterative approach minimizing $\chi^{2}$ a best set of parameters is found.

Constraints: There may be significant constraints; an example is the required balance of $\bar{s}=s$ as strangeness is produced in pairs and strangeness changing weak decays have no time to operate [91]. Such constraints can be implemented most effectively by constraints in the iteration steps; the iterative steps do not need till the very end to conserve e.g. strangeness.

Bulk properties: When our iteration has converged, we have obtained all primary particle yields; those that are measured, and all others that are, in essence, extrapolations from known to unknown. It is evident that we can use all these yields in order to compute the bulk properties of the fireball source, where the statement is exact for the conserved quantities such as net baryon number (baryons less antibaryons) and approximate for quantities where kinetic models show little modification of the value during hadronization. An example here is the number of strange quark pairs or entropy.

Discussion: The best fit is characterized by a value function, typically $\chi^{2}$ eq. (12). Depending on the complexity of the model, and the accuracy of the inherent physics picture, we can arrive at either a well converged fit, or at a poor one where $\chi^{2}$ normalized by degrees of freedom (dof) is significantly above unity. Since the objective of the SHM is the description of the data, for the case of a bad $\chi^{2}$ one must seek a more complex model. The question about analysis degeneracy also arises: are there two different SHM model variants that achieve in a systematic way as a function of reaction energy and/or collision parameters always a success? Should degeneracy be suspected, one must attempt to break degeneracy by looking at specific experimental observables, as was argued in ref. [92].

We perform SHM analysis of all "elementary" hadrons produced - that is we exclude composite light nuclei and antinuclei that in their tiny abundances may have a different production history; we will allow the data to decide what are the necessary model characteristics. We find that for all the data we study and report on in sect. 10, the result is strongly consistent with the parameter set and values associated with chemical non-equilibrium. In any case, we obtain a deeper look into the history of the expanding QGP fireball and QGP properties at chemical freeze-out temperature $T<T_{\mathrm{H}}$ and, we argue that QGP was formed. In a study of the bulk fireball properties a precise description of all relevant particle yields is needed. Detailed results of SHM analysis are presented in sect. 10. 


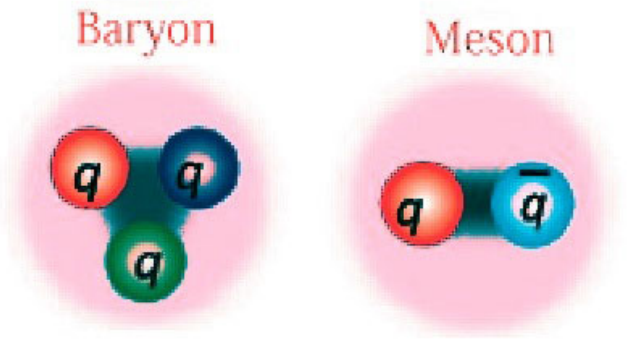

Fig. 10. Illustration of the quark bag model colorless states: baryons $q q q$ and mesons $q \bar{q}$. The range of the quantum wave function of quarks, the hadronic radius is indicated as a (pink) cloud, the color electrical field lines connect individual quarks.

\section{The concepts: Theory quark side}

\subsection{Are quarks and gluons "real" particles?}

The question to be addressed in our context is: How can quarks and gluons be real particles and yet we fail to produce them? The fractional electrical charge of quarks is a strong characteristic feature and therefore the literature is full of false discoveries. Similarly, the understanding and explanation of quark confinement has many twists and turns, and some of the arguments though on first sight contradictory are saying one and the same thing. Our present understanding requires the introduction of a new paradigm, a new conceptual context how in comparison to the other interactions the outcome of strong interactions is different.

A clear statement is seen in the September 28, 1979 lecture by T.D. Lee [93] and the argument is also presented in T.D. Lee's textbook [94]: at zero temperature quarks can only appear within a bound state with other quarks as a result of transport properties of the vacuum state, and not as a consequence of the enslaving nature of interquark forces. However, indirectly QCD forces provide the vacuum structure, hence quarks are enslaved by the same QCD forces that also provide the quark-quark interaction. Even so the conceptual difference is clear: we can liberate quarks by changing the nature of the vacuum, the modern day æther, melting its confining structure.

The quark confinement paradigm is seen as an expression of the incompatibility of quark and gluon colorelectrical fields with the vacuum structure. This insight was inherent in the work by Ken Wilson [95] which was the backdrop against which an effective picture of hadronic structure, the "bag model" was created in 1974/1975 [9699]. Each hadronic particle is a bubble [96]. Below $T_{\mathrm{H}}$, with their color field lines expelled from the vacuum, quarks can only exist in colorless cluster states: baryons $q q q$ (and antibaryons $\overline{q q q}$ ) and mesons $q \bar{q}$ as illustrated in fig. 10.

These are bubbles with the electric field lines contained in a small space domain, and the color-magnetic (spin-spin hyperfine) interactions contributing the details of the hadron spectrum [97]. This implementation of quark confinement is the so-called (MIT) quark-bag model. By imposing boundary conditions between the two vacuua,

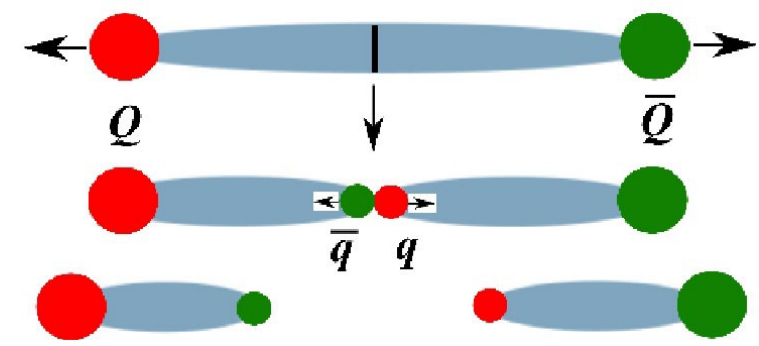

Fig. 11. Illustration of the heavy quark $Q=c, b$ and antiquark $\bar{Q}=\bar{c}, \bar{b}$ connected by a color field string. As $Q \bar{Q}$ separate, a pair of light quarks $q \bar{q}$ caps the broken field-string ends.

quark-hadron wave functions in a localized bound state were obtained; for a succinct review see Johnson [98]. The later developments which address the chiral symmetry are summarized in 1982 by Thomas [99], completing the model.

The quark-bag model works akin to the localization of quantum states in an infinite square-well potential. A new ingredient is that the domain occupied by quarks and/or their chromo-electrical fields has a higher energy density called bag constant $\mathcal{B}$ : the deconfined state is the state of higher energy compared to the conventional confining vacuum state. In our context an additional finding is important: even for small physical systems comprising three quarks and/or quark-antiquark pairs once strangeness is correctly accounted for, only the volume energy density $\mathcal{B}$ without a "surface energy" is present. This was shown by an unconstrained hadron spectrum model study [100, 101]. This result confirms the two vacuum state hypothesis as the correct picture of quark confinement, with nonanalytical structure difference at $T=0$ akin to what is expected in a phase transition situation.

The reason that in the bag model the color-magnetic hyperfine interaction dominates the color-electric interaction is due to local color neutrality of hadrons made of light quarks; the quark wave function of all light quarks fill the entire bag volume in same way, hence if the global state is colorless so is the color charge density in the bag. However, the situation changes when considering the heavy charm $c$, or bottom $b$, quarks and antiquarks. Their mass scale dominates, and their semi-relativistic wave functions are localized. The color field lines connecting the charges are, however, confined. When we place heavy quarks relatively far apart, the field lines are, according to the above, squeezed into a cigar-like shape, see top of fig. 11.

The field occupied volume grows linearly with the size of the long axis of the cigar. Thus heavy quarks interact when pulled apart by a nearly linear potential, but only when the ambient temperature $T<T_{\mathrm{H}}$. One can expect that at some point the field line connection snaps, producing a quark-antiquark pair. This means that when we pull on a heavy quark, a colorless heavy-meson escapes from the colorless bound state, and another colorless heavyantimeson is also produced; this sequence is shown from top to bottom in fig. 11. The field lines connecting the quark to its color-charge source are called a "QCD string". 
The energy per length of the string, the string tension, is nearly $1 \mathrm{GeV} / \mathrm{fm}$. This value includes the modification of the vacuum introduced by the color field lines.

For $T>T_{\mathrm{H}}$ the field lines can spread out and mix with thermally produced light quarks. However, unlike light hadrons which melt at $T_{\mathrm{H}}$, the heavy $Q \bar{Q}$ mesons (often referred as "onium states, like in charmonium $c \bar{c}$ ) may remain bound, albeit with different strength for $T>T_{\mathrm{H}}$. Such heavy quark clustering in QGP has been of profound interest: it impacts the pattern of production of heavy particles in QGP hadronization [102,103]. Furthermore, this is a more accessible model of what happens to light quarks in close vicinity of $T_{\mathrm{H}}$, where considerable clustering before and during hadronization must occur.

The shape of the heavy quark potential, and thus the stability of "onium states can be studied as a function of quark separation, and of the temperature, in the framework of lattice-QCD, showing how the properties of the heavy quark potential change when deconfinement sets in for $T>T_{\mathrm{H}}[104,105]$.

To conclude, quarks and gluons are real particles and can, for example, roam freely above the vacuum melting point, i.e. above Hagedorn temperature $T_{\mathrm{H}}$. This understanding of confinement allows us to view the quark-gluon plasma as a domain in space in which confining vacuum structure is dissolved, and chromo-electric field lines can exist. We will return to discuss further ramifications of the QCD vacuum structure in subsects. 7.2 and 7.3.

\subsection{Why do we care about lattice-QCD?}

The understanding of quark confinement as a confinement of the color-electrical field lines and characterization of hadrons as quark bags suggests as a further question: how can there be around us, everywhere, a vacuum structure that expels color-electric field lines? Is there a latticeQCD based computation showing color field lines confinement? Unfortunately, there seems to be no answer available. Lattice-QCD produces values of static observables, and not interpretation of confinement in terms of moving quarks and dynamics of the color-electric field lines.

So why care about lattice-QCD? For the purpose of this article lattice-QCD upon convergence is the ultimate authority, resolving in an unassailable way all questions pertinent to the properties of interacting quarks and gluons, described within the framework of QCD. The word lattice reminds us how continuous space-time is represented in a discrete numerical implementation on the most powerful computers of the world.

The reason that we trust lattice-QCD is that it is not a model but a solution of what we think is the foundational characterization of the hadron world. Like in other theories, the parameters of the theory are the measured properties of observed particles. In case of QED we use the Coulomb force interaction strength at large distance, $\alpha=e^{2} / \hbar c=1 / 137$. In QCD the magnitude of the strength of the interaction $\alpha_{s}=g^{2} / \hbar c$ is provided in terms of a scale, typically a mass that the lattice approach captures precisely; a value of $\alpha_{s}$ at large distance cannot be measured given the confinement paradigm.

There are serious issues that have impacted the capability of the lattice-QCD in the past. One is the problem of Fermi-statistics which is not easily addressed by classical computers. Another is that the properties we wanted to learn about depend in a decisive way on the inclusion of quark flavors, and require accurate value of the mass of the strange quark; the properties of QCD at finite $T$ are very finely tuned. Another complication is that in view of today's achievable lattice point and given the quark-, and related hadron-, scales, a lattice must be much more finely spaced than was believed necessary 30 years ago. Serious advances in numerical and theoretical methods were needed, see e.g. refs. [9,29].

Lattice capability is limited by how finely spaced lattice points in terms of their separation must be so that over typical hadron volume sufficient number is found. Therefore, even the largest lattice implemented at present cannot "see" any spatial structure that is larger than a few proton diameters, where for me: few $=2$. The rest of the Universe is, in the lattice approach, a periodic repetition of the same elementary cell.

The reason that lattice at finite temperature cannot replace models in any foreseeable future is the time evolution: temperature and time are related in the theoretical formulation. Therefore considering hadrons in a heat bath we are restricted to consideration of a thermal equilibrium system. When we include temperature, nobody knows how to include time in lattice-QCD, let alone the question of time sequence that has not been so far implemented at $T=0$. Thus all we can hope for in hot-lattice-QCD is what we see in this article, possibly much refined in understanding of internal structure, correlations, transport coefficient evaluation, and achieved computational precision.

After this description some may wonder why we should bother with lattice-QCD at all, given on one hand its limitations in scope, and on another the enormous cost rivaling the experimental effort in terms of manpower and computer equipment. The answer is simple; lattice-QCD provides what model builders need, a reference point where models of reality meet with solutions of theory describing the reality.

We have already by example shown how this works. In the previous sect. 2 we connected in several different ways the value of $T_{\mathrm{H}}$ to lattice results. It seems clear that the interplay of lattice, with experimental data and with models can fix $T_{\mathrm{H}}$ with a sufficiently small error. A further similar situation is addressed in the following subsect. 3.3 where we seek to interpret the lattice results on hot QCD and to understand the properties of the new phase of matter, quark-gluon plasma.

\subsection{What is quark-gluon plasma?}

An artist's view of Quark-Gluon Plasma (QGP), fig. 12, shows several quarks and "springy" gluons -in an image similar to fig. 10. It is common to represent gluons by 


\section{Quark-Gluon-Plasma}

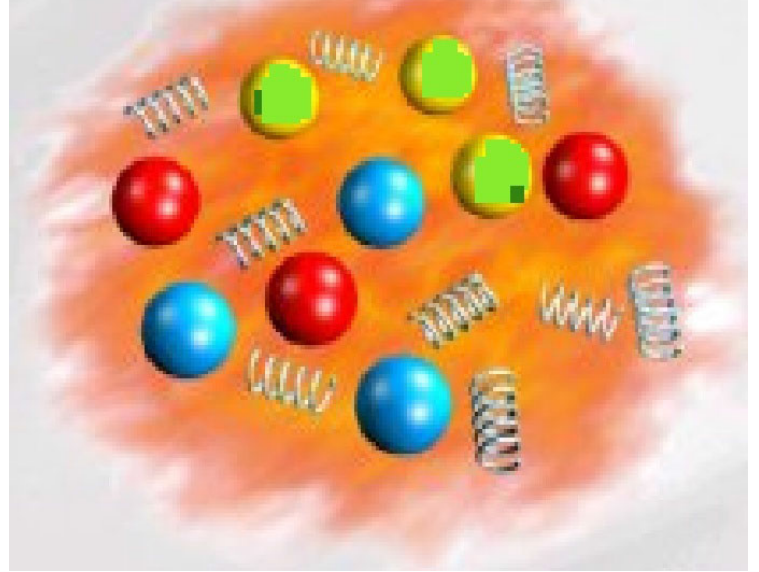

Fig. 12. Illustration of quark-gluon plasma (QGP) comprising several red, green, blue (RGB colored) quarks and springy gluons in a modified vacuum state.

springs, a historical metaphor from times when we viewed gluons as creating a force that grew at a distance so as to be able to permanently keep quarks confined. Our views of confinement evolved, but springs remain in gluon illustrations. In principle these springs are also colored: there are 9 bi-color combination, and excluding the "white" case we have 8 bi-colors of gluons. As this is hard to illustrate, these springs are gray. The domain of space comprising quarks and gluons is colored to indicate that we expect this to be a much different space domain from the surroundings.

In a nutshell, QGP in the contemporary use of the language is an interacting localized assembly of quarks and gluons at thermal (kinetic) and (close to) chemical (abundance) equilibrium. The word "plasma" signals that free color charges are allowed. Since the temperature is above $T_{\mathrm{H}}$ and thus above the scale of light quark $u, d$-mass, the pressure exhibits the relativistic Stefan-Boltzmann format,

$$
P=\left(g_{\mathrm{B}}^{*}+\frac{7}{8} g_{\mathrm{F}}^{*}\right) \frac{(\pi T)^{4}}{90 \pi^{2}}+g_{\mathrm{F}}^{* *}\left(\frac{(\pi T)^{2} \mu^{2}}{24 \pi^{2}}+\frac{\mu^{4}}{48 \pi^{2}}\right) .
$$

The stars next to degeneracy $g$ for Bosons B, and Fermions $\mathrm{F}$, indicate that these quantities are to be modified by the QCD interaction which affects this degeneracy significantly, and differently for B, F and also fort the two terms ${ }^{*} \mathrm{~F}$ vs. ${ }^{* *} \mathrm{~F}$.

In eq. (13) the traditional Stefan-Boltzmann $T^{4}$ terms, and the zero temperature limit quark-chemical potential $\mu^{4}$ term are well known and also easy to obtain by integrating the Bose/Fermi gas expressions in the respective limit. The ideal (QCD interaction $\alpha_{s}=0$ ) relativistic hot quark gas at finite $T$ including the $T^{2} \mu^{2}$ term in explicit analytical form of the expression was for the first time presented by Harrington and Yildiz in 1974 [106] in a work which has the telling title "High-Density Phase Transitions in Gauge Theories".

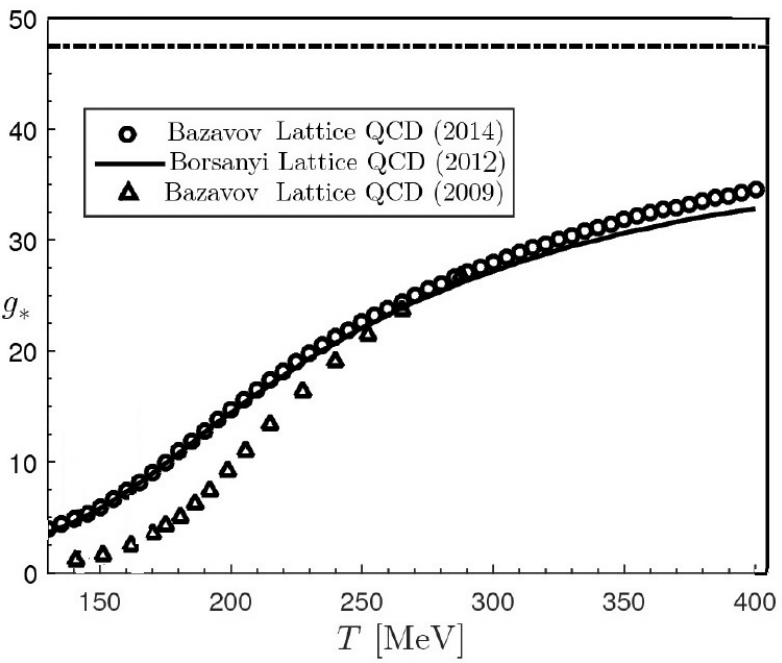

Fig. 13. The number of degrees of freedom $g_{*}$ as function of temperature $T$. The solid line includes the effect of QCD interactions as obtained within the framework of lattice QCD by Borsanyi et al. beginning in 2012 and published in 2014 (see text). Horizontal dashed line: $g=47.5$ for free quark-gluon gas.

Regarding the degeneracy factors for the ideal gases: The Boson term generalizes the usual Stefan-Boltzmann expression by an added factor $8_{c}$ for color degeneracy of gluons:

$$
g_{\mathrm{B}}=2_{s} \times 8_{c}=16 .
$$

The corresponding $T^{4}$ Fermi (quark) Stefan-Boltzmann term differs by the well known factor $7 / 8$ for each degree of freedom. We count particles and antiparticles as degrees of freedom:

$$
g_{\mathrm{F}}=2_{s} \times 2_{p} \times 3_{c} \times(2+1)_{f}=31.5,
$$

where indices stand for: $s=$ spin $(=2), p$-particle and antiparticle $(=2), c=$ color $(=3$, or $=8), f$-flavor: 2 flavors $q=u, d$ always satisfy $m_{q} \ll T$ and one flavor (strangeness $s$ ) at phase boundary satisfies $m_{s} \lesssim T$ and turns into a light flavor at high temperatures. To make sure this situation is remembered we write $(2+1)_{f}$.

The analytical and relatively simple form of the first order in $\mathcal{O}\left(\alpha_{s}\right)$ thermal QCD perturbative correction results are given in analytical format in the work of Chin in 1979 [107], and result in the following degeneracy:

$$
\begin{aligned}
g_{*} \equiv & g_{\mathrm{B}}^{*}+\frac{7}{8} g_{\mathrm{F}}^{*}=2_{s} \times 8_{c}\left(1-\frac{15 \alpha_{s}}{4 \pi}\right) \\
& +\frac{7}{8} 2_{s} \times 2_{p} \times 3_{c} \times(2+1)_{f}\left(1-\frac{50 \alpha_{s}}{21 \pi}\right) \\
g_{\mathrm{F}}^{* *}= & 2_{s} \times 2_{p} \times 3_{c} \times(2+1)_{f}\left(1-\frac{2 \alpha_{s}}{\pi}\right) .
\end{aligned}
$$

$\alpha_{s}$ is the QCD energy scale dependent coupling constant. In the domain of $T$ we consider $\alpha_{s} \simeq 0.5$, but it is rapidly decreasing with $T$. To some extent this is why in fig. 13, showing the lattice-QCD results for pressure, 
we see a relatively rapid rise of $g_{*}$ as a function of $T$, towards the indicated limit $g=47.5$ of a free gas, horizontal dashed line. In fig. 13 three results also depict the path to the current understanding of the value of $T_{\mathrm{H}}$. The initial results (triangles)were presented by Bazavov 2009 [108]; this work did not well describe the "low" temperature domain where the value of $T_{\mathrm{H}}$ is determined. This is the origin of the urban legend that $T_{\mathrm{H}} \simeq 190 \mathrm{MeV}$, and a lot of confusion.

The solid line in fig. 6 shows Borsanyi et al. 2012 [71] results presented at the Quark Matter 2012 meeting, with later formal paper comprising the same results [72]; these are the same results as we see in fig. 6 connecting at low $T$ with HRG. These results of the Wuppertal-Budapest group at first contradicted the earlier and highly cited result of [108]. However, agreement between both lattice groups was restored by the revised results of Bazavov 2014 (HotQCD Collaboration) [109].

Let us also remember that the low value of $T_{\mathrm{H}}$ we obtained at the end of subsect. 2.6 is due to the difference seen below in fig. 13 between the results of 2009, and those reported a few years later, through 2014. This difference is highly relevant and shows that hadrons melt into quarks near to $T_{\mathrm{H}}=138 \pm 12 \mathrm{MeV}$ corresponding to $4<a<4.5$ see table 2 .

As the results of lattice-QCD became reliable in the high $T>300 \mathrm{MeV}$ domain a decade ago, it became apparent that an accurate understanding of $g_{*}$ emerges [110] by taking $\mathcal{O}\left(\alpha_{s}\right)$ corrections literally and evaluating the behavior $\alpha_{s}(T)$. A more modern study of the behavior of thermal QCD and its comparison with lattice QCD is available [111-113]. The thermal QCD explains the difference between the asymptotic value $g=47.5$ and lattice results which we see in fig. 13 to be significant at the highest $T=400$ considered. In fact thermal quarks are never asymptotically free; asymptotic freedom for hot QCD matter quarks suffers from logarithmic behavior. $\alpha_{s}(T)$ drops slowly and even at the thermal end of the standard model $T \rightarrow 150,000 \mathrm{MeV}$ the QCD interaction remains relevant and $g_{*} / g \simeq 0.9$. This of course is also true for very high density cold QCD matter, a small disappointment when considering the qualitative ideas seen in the work of Collins and Perry [35].

We can conclude by looking at high $T$ domains of all these results that the state of strongly interacting matter at $T \simeq 4 T_{\mathrm{H}}$ is composed of the expected number of nearly free quarks and gluons, and the count of these particles in thermal-QCD and lattice-QCD agree. We can say that QGP emerges to be the phase of strongly interacting matter which manifests its physical properties in terms of nearly free dynamics of practically massless gluons and quarks. The "practically massless" is inserted also for gluons as we must remember that in dense plasma matter all color charged particles including gluons acquire an effective in medium mass.

It seems that today we are in control of the hot QCD matter, but what properties characterize this QGP that differ in a decisive way from more "normal" hadron matter? It seems that the safest approach in a theoretical review is to rely on theoretical insights. As the results of lattice-QCD demonstrate, the "quark-gluon plasma" is a phase of matter comprising color charged particles (gluons and quarks) that can move nearly freely so as to create ambient pressure close to the Stefan-Boltzmann limit and whose motion freezes into hadrons across a narrow temperature domain characteristic of the Hagedorn temperature $T_{\mathrm{H}}$. The properties of QGP that we check for are thus:

1) Kinetic equilibrium —allowing a meaningful definition of temperature.

2) Dominance by effectively massless particles assuring that $P \propto T^{4}$.

3) Both quarks in their large number, and gluons, must be present in conditions near chemical (yield) equilibrium with their color charge "open" so that the count of their number produces the correctly modified StefanBoltzmann constant of QCD.

\subsection{How did the name QGP come into use?}

In this article we use practically always the words QuarkGluon Plasma and the acronym QGP to describe the phase of matter made of deconfined quarks and gluons interacting according to (thermal) QCD and described in numerical lattice simulations with ever increasing accuracy. However, even today there is a second equivalent name; the series of conferences devoted to the study of quark-gluon plasma formation in laboratory calls itself "Quark Matter". In 1987 Léon Van Hove (former scientific director general of CERN) wrote a report entitled "Theoretical prediction of a new state of matter, the "quarkgluon plasma" (also called "quark matter")" [114] establishing the common meaning of these two terms.

When using Quark Matter we can be misunderstood to refer to zero-temperature limit. That is why QGP seems the preferred term. However, to begin, QGP actually meant something else. This is not unusual; quite often in physics in the naming of an important new insight older terms are reused. This phenomenon reaches back to antiquity: the early ancient Greek word "Chaos" at first meant "emptiness". The science of that day concluded that emptiness would contain disorder, and the word mutated in its meaning into the present day use.

At first QGP denoted a parton gas in the context of $p p$ collisions; Hagedorn attributes this to Bjorken 1969, but I could not find in the one paper Hagedorn cited the explicit mention of "QGP" (see chapt. 25 in [1]). Shuryak in 1978 [115] used "QGP" in his publication title addressing partons in $p p$ collisions, thus using the language in the old fashion.

Soon after "QGP" appears in another publication title, in July 1979 work by Kalashnikov and Klimov [116], now describing the strongly interacting quark-gluon thermal equilibrium matter. This work did not invent what the authors called QGP. They were, perhaps inadvertently, connecting with the term used by others in another context giving it the contemporary meaning. The results of 
Kalashnikov-Klimov agree with our eq. (16) attributed to a year earlier, July 1978, work of S.A. Chin [107] presented under the title Hot Quark Matter. This work (despite the title) included hot gluons and their interaction with quarks and with themselves.

But QGP in its new meaning already had deeper roots. Quark-star models [117] appear as soon as quarks are proposed; "after" gluons join quarks [118], within a year

- Peter Carruthers in 1973/74 [119] recognized that dense quark matter would be a quite "bizarre" plasma and he explores its many body aspects. His paper has priority but is also hard to obtain, published in a new journal that did not last.

- A theory of thermal quark matter is that of Harrington and Yidliz 1974 [106], but has no discussion of the role of gauge interaction in quantitative terms. This paper is little known in the field of RHI collisions yet it lays the foundation for the celebrated work by Linde on electroweak symmetry restoration in the early Universe [120]. There is a remarkable bifurcation in the literature: those who study the hot Universe and its early stages use the same physics as those who explore the properties of hot quarks and gluons; yet the crosscitations between the two groups are sparse.

- Collins and Perry 1975 [35] in Superdense Matter: Neutrons or Asymptotically Free Quarks propose that high density nuclear matter turns into quark matter due to weakness of asymptotically free QCD. Compared to Harrington and Yidliz this is a step back to a zerotemperature environment, yet also a step forward as the argument that interaction could be sufficiently weak to view the dense matter as a Fermi gas of quarks is explicitly made.

Following this there are a few, at times parallel developments - but this is not the place to present a full history of the field. However fragmentary, let me mention instead those papers I remember best:

- Freedman and McLerran 1976/77 [121] who address the thermodynamic potential of an interacting relativistic quark gas.

- Shuryak 1977/78 [122], writes about Theory of Hadron Plasma developing the properties of QGP in the framework of QCD.

- Kapusta 1978/79 [123] which work completes Quantum Chromodynamics at High Temperature.

- Chin 1978 [107] synthesized all these results and was the first to provide the full analytical first order $\alpha_{s}$ corrections as seen in eq. (16).

However, in none of the early thermal QCD work is the acronym "QGP", or spelled out "Quark-Gluon Plasma" introduced. So where did Kalashnikov-Klimov [116] get the idea to use it? I can speculate that seeing the work by Shuryak on Theory of Hadron Plasma they borrowed the term from another Shuryak paper [115] where he used "QGP" in his title addressing partons in $p p$ collisions. Indeed, in an aberration of credit Shuryak's $p p$ parton work is cited in $A A$ QGP context, clearly in recognition of the

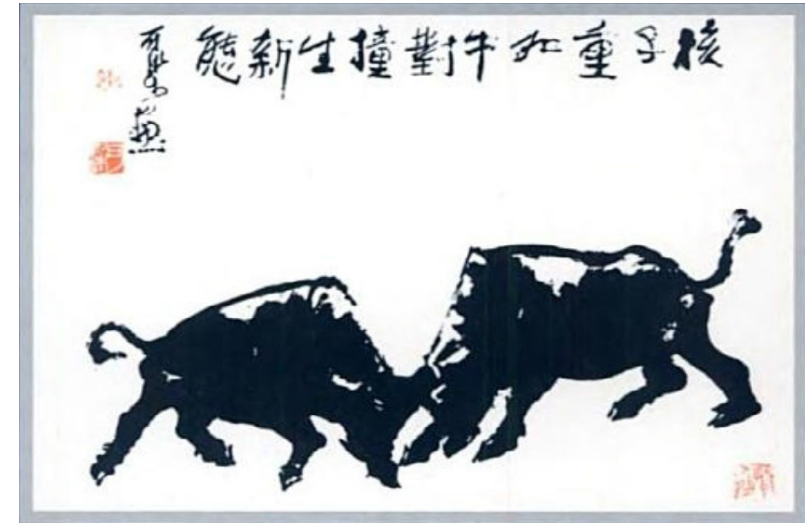

Fig. 14. Ink painting masterpiece 1986: Nuclei as Heavy as Bulls, Through Collision Generate New States of Matter by Li Keran, reproduced from open source works of T.D. Lee.

use of the QGP acronym in the title, while Shuryak's "true" QGP paper, Theory of Hadron Plasma is often not cited in this context. In his 1980 review Shuryak [124] is almost shifting to QGP nomenclature, addressing "QCD Plasma" and also uses in the text "Quark Plasma", omitting to mention "gluons" which are not established experimentally for a few more years. In this he echoes the approach of others in this period.

Having said all the above, it is clear that when "QGP" is mentioned as the theory of both hot quarks and hot gluons, we should remember Kalashnikov-Klimov [116] for as I said, the probably inadvertent introduction of this name into its contemporary use.

\section{Quark-gluon plasma in laboratory}

\subsection{How did RHI collisions and QGP come together?}

The artistic representation of RHI collisions is seen in fig. 14 -two fighting bulls. The ink masterpiece was created in 1986 by Li Keran and has been around the field of heavy ions for the past 30 years, a symbol of nascent symbiosis between science and art, and also a symbol of great friendship between T.D. Lee and Li Keran. The bulls are the heavy ions, and the art depicts the paradigm of heavy-bull(ion) collisions.

So how did the bulls aka heavy ions connect to QGP? In October 1980, I remarked in a citation" "The possible formation of quark-gluon plasma in nuclear collisions was first discussed quantitatively by S.A. Chin: Phys. Lett. B 78, 552 (1978); see also N. Cabibbo, G. Parisi: Phys. Lett. B 59, 67 (1975)". Let me refine this:

a) The pioneering insight of the work by Cabibbo and Parisi [36] is: i) to recognize the need to modify SBM to include melting of hadrons and ii) in a qualitative

3 The present day format requirement means that these words are now found in the text of ref. [15], end of 3rd paragraph below eq. (61), so that each of the two references can be cited and hyperlinked as a separate citation item. 
drawing to recognize that both high temperature and baryon density allow a phase transformation process. However, there is no mention direct or indirect in this work about "bull" collision.

b) The paper by Chin [107], of July 1978, in its ref. [7] grants the origin of the idea connecting RHI with QGP to Chapline and Kerman [125], an unpublished manuscript entitled On the possibility of making quark matter in nuclear collisions of March 1978. This paper clearly states the connection of QGP and RHI collisions that Chin explores in a quantitative fashion recomputing the QCD thermodynamic potential, and enclosing particles, quarks and gluons, in the bag-like structure, see sect. 3.1.

The preprint of Chapline and Kerman is available online at MIT [125]. It is a qualitative, mostly conceptual idea paper, a continuation of an earlier effort by Chapline and others in 1974 [126] where we read (abstract):

\section{It is suggested that very hot and dense nuclear mat- ter may be formed in a transient state in "head-on" collisions of very energetic heavy ions with medium and heavy nuclei. A study of the particles emitted in these collisions should give clues as to the nature of dense hot nuclear matter.}

At the time of the initial Chapline effort in 1974 it was too early for a mention of quark matter and heavy ions in together. Indeed, at the Bear Mountain [127] workshop in Fall 1974 the physics of the forthcoming RHI collisions was discussed in a retreat motivated by Lee-Wick [128] matter, a proposed new state of nuclear matter. These authors claim:

... the state ... inside a very heavy nucleus can become the minimum-energy state, at least within the tree approximation; in such a state, the "effective" nucleon mass inside the nucleus may be much lower than the normal value.

In presenting this work, the preeminent theorists T.D. Lee and G.C. Wick extended an open invitation to explore in relativistic heavy ion collisions the new exotic state of dense nuclear matter. This work generated exciting scientific prospects for the BEVELAC accelerator complex at Berkley. We keep in mind that there is no mention of quark matter in any document related to BEVELAC [129], nor at the Bear Mountain workshop [127]. However, the ensuing experimental search for the Lee-Wick nuclear matter generated the experimental expertise and equipment needed to plan and perform experiments in search of quark-gluon plasma [130]. And, ultimately, T.D. Lee will turn to recognize QGP as the new form of hot nuclear matter resulting, among other things, in the very beautiful painting by Li Keran, fig. 14 .

Now back to the March 1978 Chapline-Kerman manuscript: why was it never published? There are a few possible answers: a) It is very qualitative; b) In the $5 y$ run up period 1973-1978 the field of RHI collisions was dominated by other physics such as Lee-Wick. In fact at the time quarks were not part of nuclear physics which "owned" the field of heavy ions. Judging by personal experience I am not really surprised that Chapline-Kerman work was not published. Planck was dead for 30 years ${ }^{4}$. It is regrettable that once Chapline-Kerman ran into resistance they did not pursue the publication, and/or further development of their idea; instead,

a) A year later, Kerman (working with Chin who gave him the credit for the QGP-RHI connection idea in his paper), presents strangelets [131], cold drops of quark matter containing a large strangeness content.

b) And a few years later, Chapline [132] gives credit for the quark-matter connection to RHI collisions both to Chapline-Kerman [125] work, and the work of Anishetty, Koehler, and McLerran of 1980 [133]. Anishetty et al. claim in their abstract

... two hot fireballs are formed. These fireballs would have rapidities close to the rapidities of the original nuclei. We discuss the possible formation of hot, dense quark plasmas in the fireballs.

That Anishetty, Koehler, and McLerran view of RHI collision dynamics is in direct conflict with the effort of Hagedorn to describe particle production in $p p$ collisions which at the time was being adapted to the $A A$ case and presented e.g. in the QM1-report [134].

Anishetty et al. created the false paradigm that QGP was not produced centrally (as in center of momentum), a point that was corrected a few years later in 1982/83 in the renowned paper of J.D. Bjorken [135]. He obtained an analytical, one dimensional, solution of relativistic hydrodynamics that could be interpreted for the case of the RHI collision as description at asymptotically high energy of the collision events. If so, the RHI collision outcome would be a trail of energy connecting the two nuclei that naturally qualifies to be the QGP. While this replaced the Anishetty, Koehler, and McLerran "cooking nuclei, nothing in-between" picture, this new asymptotic energy idea also distracted from the laboratory situation of the period which had to deal with realistic, rather than asymptotic collision energies.

In that formative period I wrote papers which argued that the hot, dense QGP fireball would be formed due to hadron inelasticity stopping some or even all of nuclear matter in the center of momentum frame (CM). However, my referees literally said I was delusional. As history has shown (compare Chapline and Kerman) referees are not always useful. The long paper on the topic of forming QGP at central rapidity was first published 20 years later in the memorial volume dedicated to my collaborator on this project, Michael Danos [136].

Here it is good to remember that the CERN-SPS discovery story relies on the formation of a baryon-rich QGP in the CM frame of reference i.e. at "central rapidity".

\footnotetext{
${ }^{4}$ Many credit Planck with fostering an atmosphere of openness and tolerance as a publisher; certainly he did not hesitate to take responsibility for printing Einstein miraculous 1905 papers.
} 


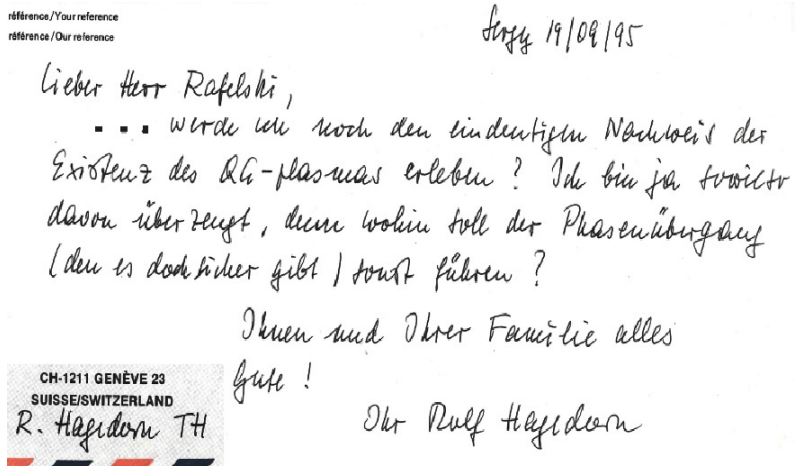

Fig. 15. Hagedorn in September 1995 awaiting QGP discovery, see text.

RHIC is in transition domain in energy, and LHC energy scale, finally and 30 years later, is near to the Bjorken "scaling" limit. The word scaling is used, as we should in a rather wide range of rapidity observe the same state of hot QGP, a claim still awaiting an experiment.

To close the topic, some regrets: an "idea" paper equivalent to ref. [125] introducing the bootstrap model of hot finite sized hadron matter and transformation into QGP in RHI collisions could have been written by Hagedorn and myself in late 1977. Hagedorn, however, desired a working model. After 10 months of telling the world about our work, and much further effort in Summer 1978 we wrote with I. Montvay a 99 page long paper [137], as well as a few months later a much evolved shorter version [28].

Only in the Spring of 1980 was Hagedorn sure we understood the SBM and the hadron melting into QGP in RHI. Of course we were looking at central rapidity i.e. CM system, quite different from the work of Anishetty et al. [133]. Hagedorn explains the time line of our and related work in his 1984 review [13]. His later point of view is succinctly represented in a letter of September 1995, fig. 15, where he says ${ }^{5}$ : “.. . can I hope to witness a proof of existence of QG plasma? I am in any case convinced of its existence, where else could the phase transition (which with certainty is present) lead?...".

\subsection{When and where was QGP discovered?}

Both CERN and BNL have held press conferences describing their experimental work. In fig. 16 a screen shot shows how CERN advertised its position in February 2000 to a wider public [138]. The document for scientists agreed to by those representing the seven CERN experiments (see the time line of CERN-SPS experiments in fig. 1) provided at the event read:

"The year 1994 marked the beginning of the CERN lead beam program. A beam of $33 \mathrm{TeV}$ (or $160 \mathrm{GeV}$ per nucleon) lead ions from the SPS now extends

\footnotetext{
${ }^{5}$ German original: ... werde ich noch den eindeutigen Nachweis der Existenz des QGP erleben? Ich bin sowieso davon überzeugt denn wohin soll der Phasenübergang (den es doch sicher gibt) sonst führen?
}

\section{CERN press office \\ New State of Matter created at CERN}

10 Feb 2000

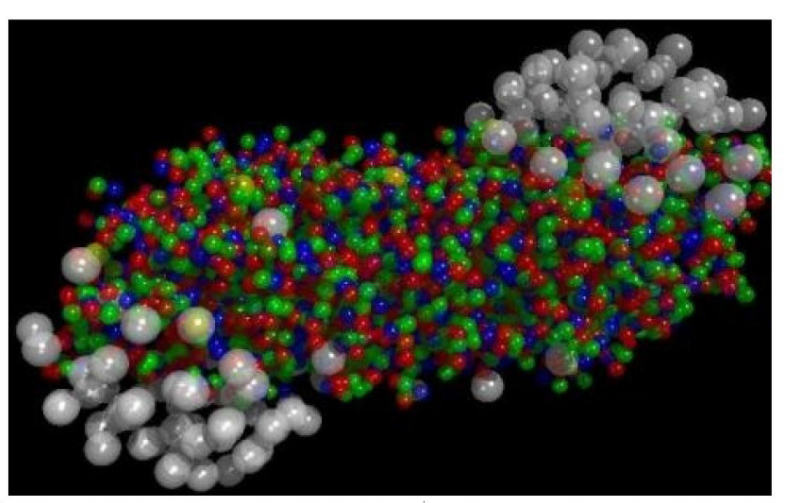

Fig. 16. The press release text: "At a special seminar on 10 February 2000, spokespersons from the experiments on CERN's Heavy Ion program presented compelling evidence for the existence of a new state of matter in which quarks, instead of being bound up into more complex particles such as protons and neutrons, are liberated to roam freely."

the CERN relativistic heavy ion program, started in the mid eighties, to the heaviest naturally occurring nuclei. A run with lead beam of $40 \mathrm{GeV}$ per nucleon in fall of 1999 complemented the program towards lower energies. Seven large experiments participate in the lead beam program, measuring many different aspects of lead-lead and lead-gold collision events: NA44, NA45/CERES, NA49, NA50, NA52/NEWMASS, WA97/NA57, and WA98. ...

Physicists have long thought that a new state of matter could be reached if the short range repulsive forces between nucleons could be overcome and if squeezed nucleons would merge into one another. Present theoretical ideas provide a more precise picture for this new state of matter: it should be a quark-gluon plasma (QGP), in which quarks and gluons, the fundamental constituents of matter, are no longer confined within the dimensions of the nucleon, but free to move around over a volume in which a high enough temperature and/or density prevails. ... (explicative in original:) A common assessment of the collected data leads us to conclude that we now have compelling evidence that a new state of matter has indeed been created, .... The new state of matter found in heavy ion collisions at the SPS features many of the characteristics of the theoretically predicted quark-gluon plasma .... In spite of its many facets the resulting picture is simple: the two colliding nuclei deposit energy into the reaction zone which materializes in the form of quarks and gluons which strongly interact with each other. This early, very dense state 
(energy density about $3-4 \mathrm{GeV} / \mathrm{fm}^{3}$, mean particle momenta corresponding to $T \approx 240 \mathrm{MeV}$ ) suppresses the formation of charmonia, enhances strangeness and begins to drive the expansion of the fireball ...."

BNL presented the following comment [139]

The CERN results are quite encouraging, says Tom Ludlam, Brookhaven's Deputy Associate Director for High-Energy and Nuclear Physics. "These results set the stage for the definitive round of experiments at RHIC in which the quark-gluon plasma will be directly observed, opening up a vast landscape for discovery regarding the nature and origins of matter."

Brookhaven's Director John Marburger congratulated CERN scientists on their achievement, stating that "piecing together even this indirect evidence of the quark-gluon plasma is a tour de force. The CERN teams have pressed their capabilities to the limit to extract these tantalizing glimpses into a new domain of matter."

Dr. Marburger was evidently expecting a better "direct evidence" to ultimately emerge. Let us look at what this may be: The turn of BNL to announce its QGP arrived 5 years later. At the April 2005 meeting of the American Physical Society, held in Tampa, Florida a press conference took place on Monday, April 18, 9:00 local time. The public announcement of this event was made April 4, 2005:

\section{EVIDENCE FOR A NEW TYPE OF NUCLEAR MATTER At the Relativistic Heavy Ion Collider (RHIC) at Brookhaven National Lab (BNL), two beams of gold atoms are smashed together, the goal being to recreate the conditions thought to have prevailed in the universe only a few microseconds after the big bang, so that novel forms of nuclear matter can be studied. At this press conference, RHIC scientists will sum up all they have learned from several years of observing the worlds most energetic collisions of atomic nuclei. The four ex- perimental groups operating at RHIC will present a consolidated, surprising, exciting new interpreta- tion of their data. Speakers will include: Dennis Ko- var, Associate Director, Office of Nuclear Physics, U.S. Department of Energy's Office of Science; Sam Aronson, Associate Laboratory Director for High Energy and Nuclear Physics, Brookhaven National Laboratory. Also on hand to discuss RHIC results and implications will be: Praveen Chaudhari, Di- rector, Brookhaven National Laboratory; represen- tatives of the four experimental collaborations at the Relativistic Heavy Ion Collider; and several the- oretical physicists.}

The participants at the press conference each obtained a "Hunting for Quark-Gluon Plasma" report, of which the cover in fig. 17 shows the four BNL experiments operating

\section{Hunting the Quark Gluon Plasma}

\author{
RESULTS FROM THE FIRST 3 YEARS AT RHIC \\ ASSESSMENTS BY THE EXPERIMENTAL COLLABORATIONS
}

April 18, 2005
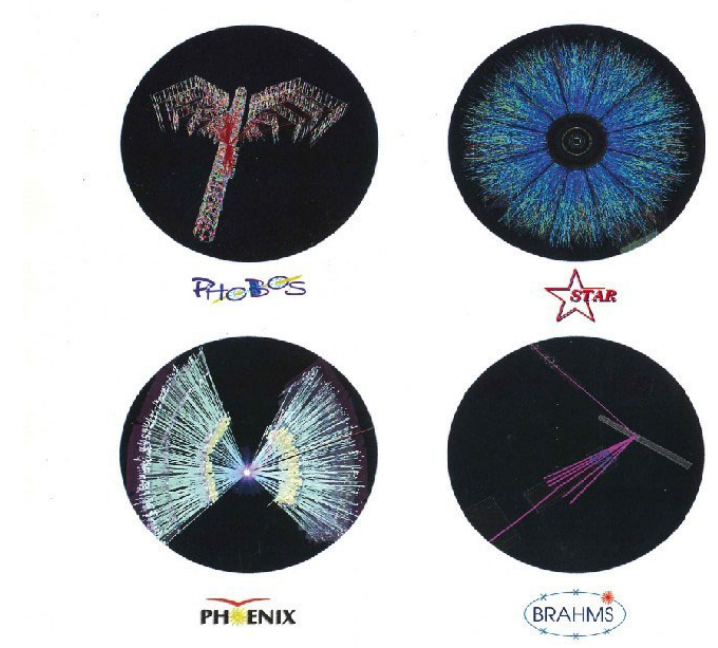

Relativistic Heavy Ion Collider (RHIO) • Brookhaven National Laboratory, Upton, NY | 1974-5000

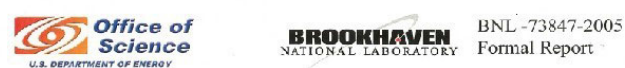

Fig. 17. The cover of the BNL-73847-2005 Formal Report prepared by the Brookhaven National Laboratory, on occasion of the RHIC experimental program press conference April 2005. The cover identified the four RHIC experiments.

at the time: BRAHMS, PHOBOS, PHENIX, and STAR, which reported on the QGP physical properties that have been discovered in the first three years of RHIC operations. These four experimental reports were later published in an issue of Nuclear Physics A [140-143].

The 10 year anniversary was relived at the 2015 RHIC \& AGS Users' Meeting, June 9-12, which included a special celebration session "The Perfect Liquid at RHIC: 10 Years of Discovery". Berndt Müller, the 2015 Brookhaven' Associate Laboratory Director for Nuclear and Particle Physics is quoted as follows [144]:

"RHIC lets us look back at matter as it existed throughout our universe at the dawn of time, before QGP cooled and formed matter as we know it, ... The discovery of the perfect liquid was a turning point in physics, and now, 10 years later, RHIC has revealed a wealth of information about this remarkable substance, which we now know to be a QGP, and is more capable than ever of measuring its most subtle and fundamental properties."

An uninvolved scientist will ask: "Why is the flow property of QGP: a) Direct evidence of QGP and b) Worth full scientific attention 15 years after the new phase of matter was announced for the first time?" Berndt Müller answers for this article:

Nuclear matter at "room temperature" is known to behave like a superfluid. When heated the nuclear 
fluid evaporates and turns into a dilute gas of nucleons and, upon further heating, a gas of baryons and mesons (hadrons). But then something new happens; at $T_{\mathrm{H}}$ hadrons melt and the gas turns back into a liquid. Not just any kind of liquid. At RHIC we have shown that this is the most perfect liquid ever observed in any laboratory experiment at any scale. The new phase of matter consisting of dissolved hadrons exhibits less resistance to flow than any other substance known. The experiments at RHIC have a decade ago shown that the Universe at its beginning was uniformly filled with a new type of material, a super-liquid, which once Universe cooled below $T_{\mathrm{H}}$ evaporated into a gas of hadrons.

Detailed measurements over the past decade have shown that this liquid is a quark-gluon plasma; i.e. matter in which quarks, antiquarks and gluons flow independently. There remain very important questions we need to address: What makes the interacting quark-gluon plasma such a nearly perfect liquid? How exactly does the transition to confined quarks work? Are there conditions under which the transition becomes discontinuous first-order phase transition? Today we are ready to address these questions. We are eagerly awaiting new results from the upgraded STAR and PHENIX experiments at RHIC.

\subsection{How did the SPS-QGP announcement withstand the test of time?}

It is impossible to present in extensive manner in this review all the physics results that have driven the SPS announcement, and I will not even venture into the grounds of the RHIC announcement. I will focus here instead on what I consider my special expertise, the strangeness signature of QGP. The events accompanying the discovery and development of strangeness signature of QGP more than 30 years ago have been reported [18], and the first extensive literature mention of strangeness signature of QGP from 1980 is found in ref. [15].

So, what exactly is this signature? The situation is illustrated in fig. 18 and described in detail in ref. [16]. In the center of the figure we see thermal QCD based strangeness production processes. This thermal production dominates the production occurring in first collision of the colliding nuclei. This is unlike heavier flavors where the mass threshold $2 m_{Q} \gg T, Q=c, b$. Strange quark pairs: $s$ and antiquarks $\bar{s}$, are found produced in processes dominated by gluon fusion [145]. Processes based on light quark collisions contribute fewer $s \bar{s}$-pairs by nearly a factor 10 [146]. When $T \geq m_{s}$ the chemical equilibrium abundance of strangeness in QGP is similar in abundance to the other light $u$ and $d$ quarks [15].

Even for the gluon fusion processes enough lifespan of QGP is needed to reach the large abundance of strange quark pairs in chemical equilibrium. The lifespan of the

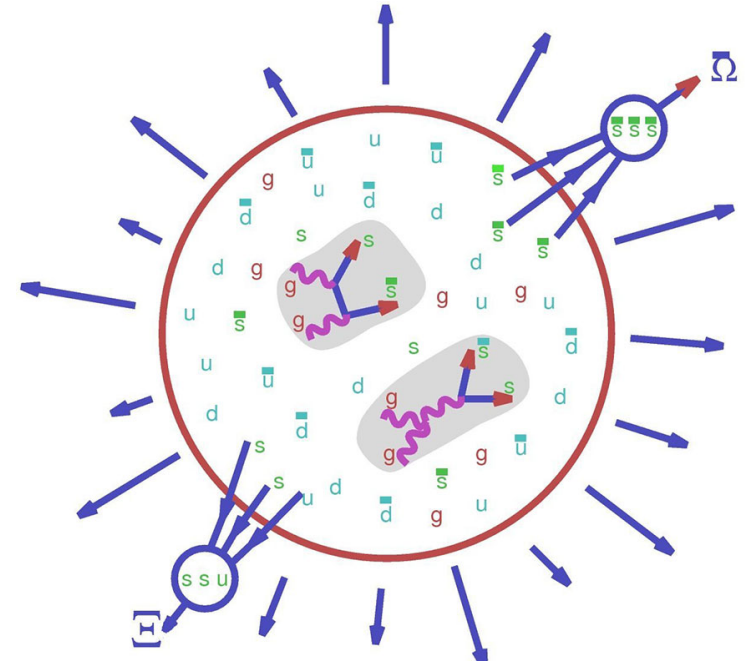

Fig. 18. Multistrange (anti)baryons as signature of QGP, see text for further discussion.

QGP fireball increases as the collision volume increases and/or the energy increases. Since the gluon fusion $G G \rightarrow$ $s \bar{s}$ dominates quark flavor conversion $q \bar{q} \rightarrow s \bar{s}$ the abundance of strangeness is signature of the formation of a thermal gluon medium.

Of course we need to ask, how come there is a gluon medium at SPS energy scale? In the cascade evolution model one finds that gluons are in general the first to equilibrate in their number and momentum distribution. Equilibration means entropy $S$ production, a topic of separate importance as $S$ production is proceeding in temporal sequence other hadronic observables of QGP, and how entropy is produced remains today an unresolved question, see subsect. 5.2 .

The gluon based processes are driving the equilibration of quarks and antiquarks; first light $q=u, d$, next the slightly massive $s$ and also some thermal evolution of charm is possible. Strangeness evolves along with the light $(u, \bar{u}, d, \bar{d})$ quarks and gluons $G$ until the time of hadronization, when these particles seed the formation of hadrons observed in the experiment. In QGP, $s$ and $\bar{s}$ can move freely and their large QGP abundance leads to unexpectedly large yields of particles with a large $s$ and $\bar{s}$ content $[147,148]$, as is illustrated exterior of the QGP domain in fig. 18 .

A signature of anything requires a rather background free environment, and a good control of anything that is there as no signature is background free. There are ways two other than QGP to make strange antibaryons:

I) Direct production of complex multistrange (anti)baryons is less probable for two reasons:

1) When new particles are produced in a color string breaking process, strangeness is known to be produced less often by a factor 3 compared to lighter quarks.

2) The generation of multistrange content requires multiple such suppressed steps. 


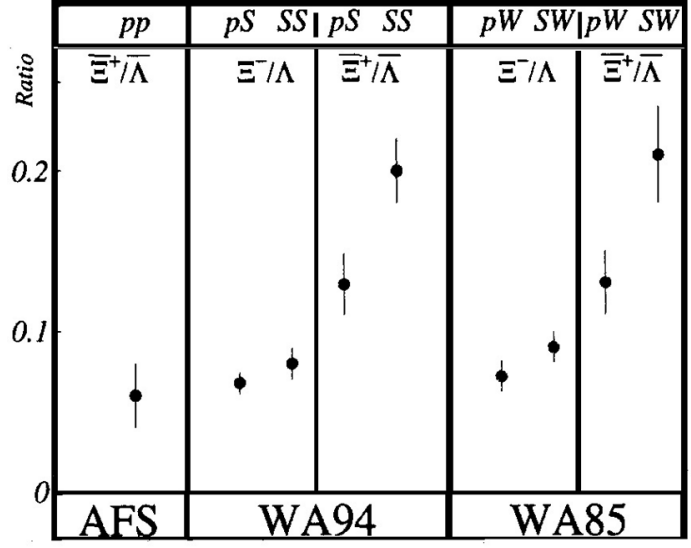

Fig. 19. Results obtained at the CERN-SPS $\Omega^{\prime}$-spectrometer for $\Xi / \Lambda$-ratio in fixed target $\mathrm{S}-\mathrm{S}$ and $\mathrm{S}-\mathrm{Pb}$ at $200 A \mathrm{GeV} / c$; results from the compilation presented in ref. [150] adapted for this report.

Thus the conclusion is that with increasing strangeness content the production by string processes of strange hadrons is progressively more suppressed.

II) Hadron-hadron collisions can redistribute strangeness into multistrange hadrons. Detailed kinetic model study shows that the hadron-reaction based production of multistrange hadrons is rather slow and requires time that exceeds collision time of RHI collisions significantly. This means that both $\Xi, \bar{\Xi}$ and $\Omega, \bar{\Omega}$ are in their abundance signatures of QGP formation and hadronization, for further details see refs. [16,55,2].

Léon Van Hove, the former DG (1976-1980), characterized the strange antibaryon signature after hearing the reports $[147,148]$ as follows [149]:

In the "Signals for Plasma" section: ... implying (production of) an abnormally large antihyperon to antinucleon ratio when plasma hadronizes. The qualitative nature of this prediction is attractive, all the more so that no similar effect is expected in the absence of plasma formation.

Given this opinion of the "man in charge", strange antibaryons became the intellectual cornerstone of the experimental strangeness program carried out at the CERN SPS, see fig. 1. Thus it was no accident that SPS research program included as a large part the exploration of the predicted strange (anti)baryon enhancement. We see this on left in fig. 1 noting that "hadrons" include of course (multi)strange hadrons and strange antibaryons.

In $A A$ collisions at the CERN-SPS $\Omega^{\prime}$-spectrometer, the production of higher strangeness content baryons and antibaryons was compared to lower strangeness content particles, $\Xi / \Lambda$ and $\bar{\Xi} / \bar{\Lambda}$. These early SPS experiments published in 1997 clearly confirmed the QGP prediction in a systematic fashion, as we see in the 1997 compilation of the pertinent experimental WA85 and WA94 results by Antinori [150], see fig. 19. Given the systematic multiple observable 3 s.d. agreement of experiment with the model predictions, I saw this result as first and clear experimental

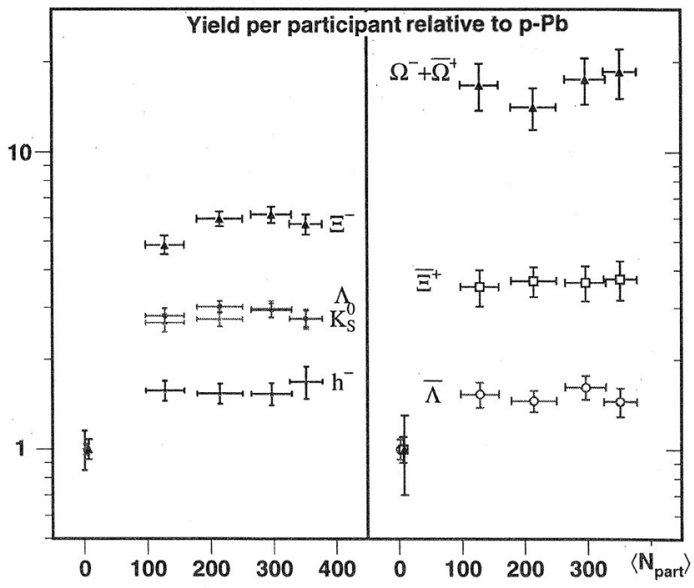

Fig. 20. Results obtained by the CERN-SPS NA57 experiment (former $\Omega^{\prime}$-spectrometer WA85 and WA94 team) for multistrangeness enhancement at mid-rapidity $\left|y_{\mathrm{CM}}\right|<0.5$ in fixed target $\mathrm{Pb}-\mathrm{Pb}$ collisions at $158 \mathrm{AGeV} / \mathrm{c}$ as a function of the mean number of participants $\left\langle N_{\text {part }}\right\rangle$, from ref. [151].

evidence of QGP obtained by the experiment-line WA85 and WA94 designed to discover QGP.

In these experiments WA85 and WA94 (see fig. 1) the sulfur ions (S) at $200 \mathrm{AGeV}$ hit stationary laboratory targets, S, W (tungsten), respectively, with reference date from $p p$ (AFS-ISR experiment at CERN) and $p$ on $\mathrm{S}$ shown for comparison. The $\Xi / \Lambda$ and $\bar{\Xi} / \bar{\Lambda}$ ratio enhancement rises with the size of the reaction volume measured in terms of target $A$, and is larger for antimatter as compared to matter particles. Looking at fig. 19, the effect is systematic, showing the QGP predicted pattern $[15,16$, $55,2]$.

The "enhancement" results obtained by the same group now working in CERN North Area for the top SPS energy $\mathrm{Pb}$ (lead) beam of $156 \mathrm{AGeV}$ as published in 1999 by Andersen (NA57 Collaboration) [151] is shown in fig. 20. On the right hadrons made only of quarks and antiquarks that are created in the collision are shown. On the left some of the hadron valence quarks from matter can be brought into the reaction volume.

The enhancement in production of higher strangeness content baryons and antibaryons in $A A$ collisions increases with the particle strangeness content. To arrive at this result, the "raw" $A A$ yields are compared with reference $p p$-, $p A$-reaction results and presented per number of "participants" $\left\langle N_{\text {part }}\right\rangle$ obtained from geometric models of reaction based on energy and particle flows. We will discuss this in subsect. 9.3. The number of collision participants for all data presented in fig. 20 is large, greater than 100, a point to remember in further discussion.

We see that production of hadrons made entirely from newly created quarks are up to 20 times more abundant in $A A$-reactions when compared to $p A$ reference measurement. This enhancement falls with decreasing strangeness content and increasing contents of the valence quarks which are brought into collision. These reference results at yield ratio "1" provide the dominant error measure. 


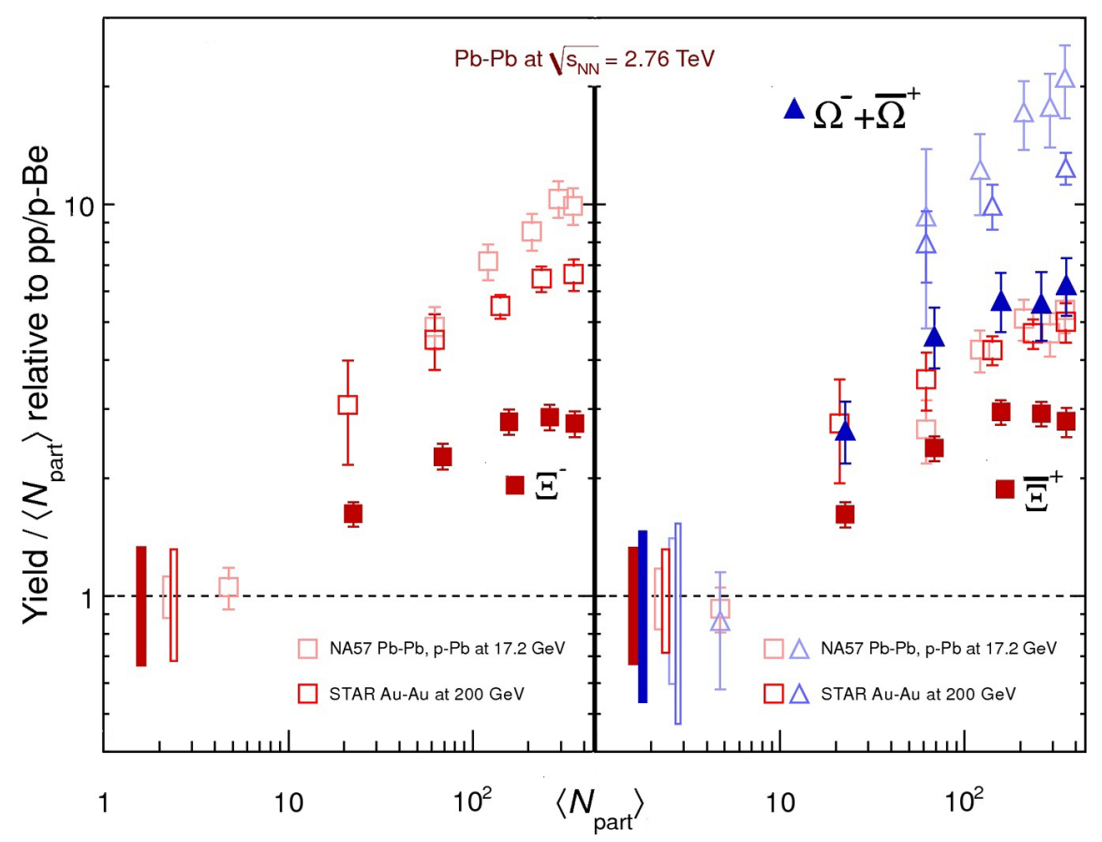

Fig. 21. Enhancements of $\Xi^{-}, \bar{\Xi}^{+}, \Omega^{-}+\bar{\Omega}^{+}$in the rapidity range $\left|y_{\mathrm{CM}}\right|<0.5$ as a function of the mean number of participants $\left\langle N_{\text {part }}\right\rangle$ : LHC-ALICE: full symbols; RHIC-STAR and SPS-NA57: open symbols. The LHC reference data use interpolated in energy $p p$ reference values. Results at the dashed line (at unity) indicate statistical and systematic uncertainties on the $p p$ or $p$ Be (at SPS) reference. Error bars on the data points represent the corresponding uncertainties for all the heavy-ion measurements. Results presented and compiled in ref. [153].

The pattern of enhancement follows the QGP prediction and is now at a level greater than 10 s.d.. There is no known explanation of these results other than QGP. This is also the largest "medium effect" observed in RHI collision experiments.

These discoveries are now all more than 15 years old. They have been confirmed by further results obtained at SPS, at RHIC, and at the LHC. The present day experimental summary is shown in fig. 21. We see results obtained by the collaborations:

SPS: NA57 for collision energy $\sqrt{s_{\mathrm{NN}}}=17.2 \mathrm{GeV}$ (lighter open symbols).

RHIC: STAR for collision energy $\sqrt{s_{\mathrm{NN}}}=200 \mathrm{GeV}$ (darker open symbols).

$L H C$ : Alice for collision energy $\sqrt{s_{\mathrm{NN}}}=2760 \mathrm{GeV}$ (filled symbols).

These results span a range of collision energies that differ by a factor 160 and yet they are remarkably similar.

Comparing the results of fig. 21 with those seen in fig. 20 we note that $\left\langle N_{\text {part }}\right\rangle$ is now on a logarithmic scale: the results of fig. 20 which show that the enhancement is volume independent are in fig. 21 compressed to a relatively small domain on the right in both panels. The SPS-NA57 results in fig. 21 are in agreement with the 1999 "high" participant number results shown in fig. 20.

The rise of enhancement which we see in fig. 21 as a function of the number of participants $2<\left\langle N_{\text {part }}\right\rangle<80$ reflects on the rise of strangeness content in QGP to its chemical equilibrium abundance with an increase in volume and thus lifespan of QGP fireball. It is not surprising that the enhancement at SPS is larger than that seen at RHIC and LHC, considering that the reference yields play an important role in this comparison. Especially the high energy LHC $p p$ reactions should begin to create space domains that resemble QGP and nearly achieve the degree of chemical strangeness equilibration that could erase the enhancement effect entirely.

The study of the $\phi(s \bar{s})$ abundance and enhancement corroborates these findings [152]. The importance in the present context is that while $\phi(s \bar{s})$ by its strangeness connects to $\Xi^{-}(s s d), \bar{\Xi}, \phi(s \bar{s})$ is a net-strangeness free particle. Therefore if it follows the pattern of enhancement established for $\Xi, \bar{\Xi}$ this confirms strangeness as being the quantity that causes the effect. For some of my colleagues, these year 2008 results were the decisive turning point to differentiate the strangeness effect from the effect associated with the source volume described in the closing discussion of ref. [15]. Those reading more contemporary literature should note that this volume source effect has been rediscovered three times since, and at some point in time was called "canonical suppression".

The reader should also consult subsect. 10.1, where it is shown that QGP formation threshold for $\mathrm{Pb}-\mathrm{Pb}$ collisions is found at about $1 / 4$ of the $156 \mathrm{AGeV}$ projectile energy, and that the properties of physical QGP fireball formed at SPS are just the same, up to volume size, when SPS results are compared to RHIC, and with today data from LHC. Today, seen across energy, participant number, and type of hadron considered, there cannot be any doubt that 
the source of enhancement is the mobility of quarks in the fireball, with the specific strangeness content showing gluon based processes.

Recall, in February 2000 in the snap of the QGP announcement event, the highly influential Director of BNL, Jeff Marburger ${ }^{6}$ called these NA57 results and other CERN-ion experimental results, I paraphrase the earlier year 2000 precise quote: "pieced together indirect glimpse of QGP". Today I would respond to this assessment as follows: the NA57 results seen in fig. 20 and confirmed in past 15 years of work, see fig. 21 are a direct, full panoramic sight of QGP, as good as one will ever obtain. There is nothing more direct, spectacular, and convincing that we have seen as evidence of QGP formation in RHI collision experiments.

\section{The RHI physics questions of today}

\subsection{How is energy and matter stopped?}

We arrange to collide at very high, relativistic energies, two nuclei such as lead $(\mathrm{Pb})$ or gold $(\mathrm{Au})$, having each about $12 \mathrm{fm}$ diameter. In the rest frame of one of the two nuclei we are looking at the other Lorentz-contracted nucleus. The Lorentz contraction factor is large and thus what an observer traveling along with each nucleus sees approaching is a thin, ultra dense matter pancake. As this pancake penetrates into the other nucleus, there are many reactions that occur, slowing down projectile matter.

For sufficiently high initial energy the collision occurs at the speed of light $c$ despite the loss of motion energy. Hence each observer comoving with each of the nuclei records the interaction time $\tau$ that a pancake needs to traverse the other nucleus. The geometric collision time thus is $c \tau^{0}=12 \mathrm{fm}$ as measured by an observer comoving with one of the nuclei. Thus if you are interested like Anishetty et al. [133] in hot projectile and target nuclei there is no doubt this is one of the outcomes of the collision.

An observer in the center of momentum (CM) frame can determine the fly-by time that two nuclei need to pass each other should they miss to hit: this is $\tau^{0} / \gamma$, where $\gamma$ is the Lorentz-factor of each of the nuclei with respect to CM frame. This time is, in general, very short and even if nuclei were to touch in such short time very little could happen. The situation changes if we model this like a collision of the two bulls of Li Keran and T.D. Lee. Once some of the energy (and baryon number) of two nuclei has slowed down to rest in CM, the clocks of both "slowed" bulls tick nearly at the same speed as the clock comoving with CM frame - for the stopped energy and baryon number the lifespan of the fireball is again quite large.

But how do we stop the bulls or at least some of their energy? The answer certainly depends on the energy regime. The lower is the energy of the bulls, the less we need to worry; the pancakes are not thin and one can try

\footnotetext{
6 Jeff Marburger was a long term Presidential Science Advisor, President of Stony Brook campus of the NY State University System, Director of BNL.
}

to make parton-collision cascade to describe the physics case, see e.g. Geiger-Sriwastava $[154,158,159]$ for SPS energy range. The use of these methods for RHIC or even LHC energies looks less convincing [155].

To put the problem in perspective, we need a way to concentrate entropy so that a thermal state can rapidly arise. Beginning with the work of Bjorken [135] a formation time is introduced, which is more than an order of magnitude shorter compared to $\tau^{0}$. It is hard to find tangible experimental evidence which compels a choice such as $0.5 \mathrm{fm} / c$, and theory models describing this stage are not fully convincing. A model aims to explain how as a function of collision energy and centrality the easy to observe final entropy (hadron multiplicity) content arises. For some related effort see review work of the Wernergroup [156] and Iancu-Venugopalan [157].

To summarize, in the "low" energy regime of SPS we can try to build a parton cascade model to capture the essence of heavy-ion collision dynamics $[158,159]$. The understanding of the initial "formation" of QGP as a function of collision energy and the understanding of the mechanism that describe energy and baryon number stopping remains one of the fundamental challenges of the ongoing theoretical and experimental research program.

\subsection{How and what happens, allowing QGP creation?}

In the previous subsect. 5.1 we addressed the question how the energy and baryon number is extracted from fast moving nuclei. In this section the added challenge is, how is the entropy produced that we find in the fireball? While in some solutions of the initial state formation in RHI collisions these two topics are confounded, these are two different issues: stopping precedes and is not the same as abundant entropy production.

For many the mechanism of fast, abundant entropy formation is associated with the breaking of color bonds, the melting of vacuum structure, and the deconfinement of quarks and gluons. How exactly this should work has never been shown: Among the first to address a parton based entropy production quantitatively within a kinetic collision model was Klaus Geiger [158,159] who built computer cascade models at parton level, and studied thermalization as a collision based process.

In order to understand the QGP formation process a solution of this riddle is necessary. There is more to entropy production: it controls the kinetic energy conversion into material particles. The contemporary wisdom how to describe the situation distinguishes several reaction steps in RHI collision:

1) Formation of the primary fireball; a momentum equipartitioned partonic phase comprising in a limited space-time domain, speaking in terms of orders of magnitude, almost the final state entropy.

2) The cooking of the energy content of the hot matter fireball towards the particle yield (chemical) equilibrium in a hot perturbative QGP phase.

3) Expansion and evaporation cooling towards the temperature phase boundary. 
4) Hadronization; that is, combination of effective and strongly interacting $u, d, s, \bar{u}, \bar{d}$ and $\bar{s}$ quarks and anti-quarks into the final state hadrons, with the yield probability weighted by accessible phase space.

It is the first step that harbors a mystery.

The current textbook wisdom is that entropy production requires the immersion of the quantum system in a classical environment. Such an environment is not so readily available for a RHI collision system that has a lifespan of below $10^{-22} \mathrm{~s}$ and a size less than $1 / 10000$ of atomic size. For a year 2011 review on entropy production during the different stages of RHI collision see ref. [160]. The search for a fast entropy generating mechanism continues, see for example ref. [161].

So what could be a mechanism of rapid entropy formation? Consider the spontaneous pair production in presence of a strong field: the stronger is the field the greater is the rate of field conversion into particles. One finds that when the field strength is such that it is capable of accelerating particles with a unit strength critical acceleration, the speed of field decay into particle pairs is such that a field filled state makes no sense as it decays too fast [162]. For this reason there is an effective limit to the strength of the field, and forces capable to accelerate particles at critical limit turn the field filled space into a gas of particles.

The conversion of energy stored in fields into particles, often referred to, in the QCD context, as the breaking of color strings, must be an irreversible process. Yet the textbook wisdom will assign to the time evolution pure quantum properties, and in consequence, while the complexity of the state evolves, it remains "unobserved" and thus a pure state with vanishing entropy content. Intuitively, this makes little sense. Thus the riddle of entropy production in RHI collisions which involve an encounter of two pure quantum states and turns rapidly into state of large entropy carried by many particles maybe related to our poor formulation of quantum processes for unstable critical field filled states decaying into numerous pairs.

However, the situation may also call for a more fundamental revision of the laws of physics. The reason is that our understanding is based in experience, and we really do not have much experience with critical acceleration conditions. When we study acceleration phenomena on microscopic scale, usually these are very small, even in principle zero. However, in RHI collisions when we stop partons in the central rapidity region we encounter the critical acceleration, an acceleration that in natural units is unity and which further signals a drastic change in the way fields and particles behave. The framework of physical laws which is based on present experience may not be sufficiently complete to deal with this situation and we will need to increase the pool of our experience by performing many experiments involving critical forces.

To conclude: a) The measurement of entropy production is relatively straightforward as all entropy produced at the end is found in newly produced particles; b) The QGP formation presents an efficient mechanism for the conversion of the kinetic energy of the colliding nuclei into particles in a process that is not understood despite many years of effort; c) Exploration and understanding of the principles that lead to the abundant formation of entropy in the process of QGP formation in RHI collisions harbors potential opportunity to expand the horizons of knowledge.

\subsection{Non-equilibrium in fireball hadronization}

Heavy flavor production cross sections, in lowest order in coupling constant, scale according to $\sigma \propto \alpha_{s}^{2} / m^{2}$. Considering a smaller (running) coupling, and a much larger mass of e.g. heavy quarks $c, b$, we obtain a significant reduction in the speed of thermal QGP production reactions. For charm and bottom, contribution for thermal production depends on the profile of temperature but is very likely negligible, and for charm it is at the level of a few percent. Conversely, light quarks equilibrate rather rapidly compared to the even more strongly self coupled gluons and in general can be assumed to follow and define QGP matter properties.

Heavy quark yields originate in the pre-thermal parton dynamics. However, heavy quarks may acquire through elastic collisions a momentum distribution characteristic of the medium, providing an image of the collective dynamics of the dense quark matter flow. Moreover, the question of yield evolution arises, in particular with regard to annihilation of heavy flavor in QGP evolution.

Our "boiling" QGP fireball is not immersed in a bath. It is expanding or, rather, exploding into empty space at a high speed. This assures that the entropy $S \propto V$ is not decreasing, but increasing, in consideration of internal collisions which describe the bulk viscosity. The thermal energy content is not conserved since the sum of the kinetic energy of expanding motion, and thermal energy, is conserved. Since the positive internal pressure of QGP accelerates the expansion into empty space, an explosion, the thermal energy content decreases and the fireball cools rapidly.

In this dynamical evolution quark flavors undergo chemical freeze-out. The heavier the quark, the earlier the abundance freeze-out should occur. Charm is produced in the first collisions in the formative stage of QGP. The coupling to thermal environment is weak. As ambient temperature drops the charm quark phase space given its mass drops rapidly. The quantum Fermi phase space distribution which maximizes the entropy at fixed particle number is $[163,164]$

$$
\begin{aligned}
n_{\mathrm{F}}(t) & =\frac{1}{\gamma^{-1}(t) \mathrm{e}^{(E \mp \mu) / T(t)}+1}, \\
\frac{\mathrm{d}^{6} N_{\mathrm{F}}}{\mathrm{d}^{3} p \mathrm{~d}^{3} x} & =\frac{g}{(2 \pi)^{3}} n_{\mathrm{F}}, \quad E=\sqrt{p^{2}+m^{2}},
\end{aligned}
$$

where $g$ is the statistical degeneracy, and the chemical non-equilibrium fugacity (phase space occupancy) $\gamma(t)$ is the same for particles and antiparticles while the chemical potential $\mu$ describes particle-antiparticle asymmetry, and changes sign as indicated. Our $\mu$ is "relativistic" chemical potential. In the non-relativistic limit $\mu \equiv m+\mu_{\mathrm{nr}}$ such 
that $m$ implicit in $E$ cancels out for particle but turns to a $2 m / T$ suppression for the antiparticles. Note that independent of the values of all parameters, $n_{\mathrm{F}} \leq 1$ as required.

The integral of the distribution eq. (17) provides the particle yield. When addressing SHARE phase space properties in subsect. 9.4, we will inspect the more exact result, here we consider the Boltzmann non-relativistic limit suitable for heavy quarks $(c, b)$

$$
\begin{aligned}
N & =\frac{g V T^{3}}{2 \pi^{2}} \gamma \mathrm{e}^{ \pm \mu / T} x^{2} K_{2}(x), \quad x=\frac{m}{T} \\
& \rightarrow g V(m T / 2 \pi)^{3 / 2} \gamma \mathrm{e}^{-(m \mp \mu) / T} .
\end{aligned}
$$

$T(t)$ is time dependent because the system cools. Let us look at the case $\mu=0$, appropriate for physics at LHC and, in the context of present discussion, also a good approximation at RHIC.

Considering charm abundance, in QGP chemical equilibrium $\gamma^{\mathrm{QGP}}(t) \rightarrow 1$. However, we recall that charm froze out shortly after first collisions. Therefore the value of $\gamma_{c}^{\mathrm{QGP}}(t)$ in eq. (19) is established by need to preserve the total charm pair number $N_{c}=$ const. The exponential factor $m / T$ changes from about 2 to 8 near to hadronization. Thus for prescribed yields at LHC and RHIC it is likely that $\gamma_{c}^{\mathrm{QGP}}(t)>1$. More generally there is nobody who disagrees with the need to have $\gamma_{c}^{\mathrm{QGP}} \neq 1$. $\gamma_{c}^{\mathrm{QGP}}=1$ is an accidental condition. We have established that charm, and for the very same reason, bottom flavor, cannot be expected to emerge in chemical equilibrium abundance at hadronization.

A QGP filled volume at high $T$ cooks up a high content of strangeness pairs, in essence as many as there are of each light flavor $u, d$; in plasma strangeness suppression disappears; the Wroblewski suppression factor [178] (see also next subsection) is therefore close to unity. As plasma evolves and cools at some relatively low temperature the yield of strangeness freezes-out, just like it did for charm (and bottom) at higher value of $T$.

In earlier discussion we have assumed that in QGP strangeness will follow the evolution in its pair abundance, and always be in chemical equilibrium in the fireball. This tacit assumption is not supported by kinetic theory for $T<T_{s} \simeq 180 \mathrm{MeV}$; however for such low value of $T$ the systematic error of perturbative QCD is large, thus we really do not know where approximately strangeness pair yield freezes out. We must introduce a pair fugacity parameter aside of charm also for strangeness and we now have $\gamma_{b, c, s}^{\mathrm{QGP}} \neq 1$. The phase space size of strangeness on the hadron side is smaller so once strangeness emerges one must expect that a relatively large value could be measured.

So what about $\gamma_{u, d}$ ? If the evolution as a function of $T$ of the fireball properties is smooth as lattice computation suggests, then the strongly coupled light quarks and gluons are defining the QGP properties and, remain in equilibrium ... really? The flaw in this argument is that only quarks define final hadrons. Thus gluons transform into quark pairs feeding additional mesons and baryons in that way and helping preserve entropy content. Thus gluon dissolution into additional hadrons assures that the light quark phase space occupancies as measured in terms of observed hadron abundances should show $\gamma_{u, d}^{\mathrm{HG}}>\gamma_{u, d}^{\mathrm{QGP}}>1$.

The introduction hadron-side of phase space occupancy $\gamma_{s}[65]$ and later $\gamma_{u, d}[79]$ into the study of hadron production in the statistical hadronization approach has been challenged. However there was no scientific case, challenges were driven solely by an intuitive argument that in RHI collisions at sufficiently high reaction energy aside of thermal, also chemical equilibrium is reached. One of the objectives of this review is to explain why this intuition is wrong when QGP is formed.

Note further that there is a difference between an assumption and the demonstration of a result. All know that to make a proof one generally tries to show a contrary behavior and arrives at a contradiction: in this case one starts with $\gamma_{s, u, d} \neq 1$ and shows that results are right only for $\gamma_{s, u, d} \rightarrow 1$. However, we will see in sect. 10 that results are right when $\gamma_{s, u, d} \neq 1$ and we show by example in subsect. 10.3 how the urban legend "chemical equilibrium works" formed relying on a set of errors and/or omissions.

The question about chemical non-equilibrium conditions has to be resolved so that consensus can emerge about the properties of the hadronizing QGP drop, and the mechanisms and processes that govern the hadronization process.

\section{How is the experimental study of QGP continuing today?}

Today RHI collisions and QGP is a research field that has grown to be a large fraction of nuclear science research programs on several continents. A full account of methods, ongoing experiments, scheduled runs, future plans including the development of new experimental facilities is a separate review that this author cannot write. The question how to balance presenting "nothing", with "everything", is never satisfactorily soluble. The selection of the following few topics is made in support of a few highlights of greatest importance to this review.

\subsection{Short survey of recent QGP probes and results}

A short list of contemporary QGP probes and results includes:

\section{Strangeness and other soft hadrons}

This cornerstone observable of QGP is a topic of personal expertise of the author and is addressed elsewhere and at length in these pages. The following is a brief summary: Strangeness, the lightest unstable quark flavor, appears in $p p$ collisions with an abundance that is about a factor 2.5-3 below that of each light quark flavor; this is the mentioned "Wroblewski" ratio [178]. It is natural to expect that in a larger physical $A A$ collision system additional scattering opportunity among all particles creates 


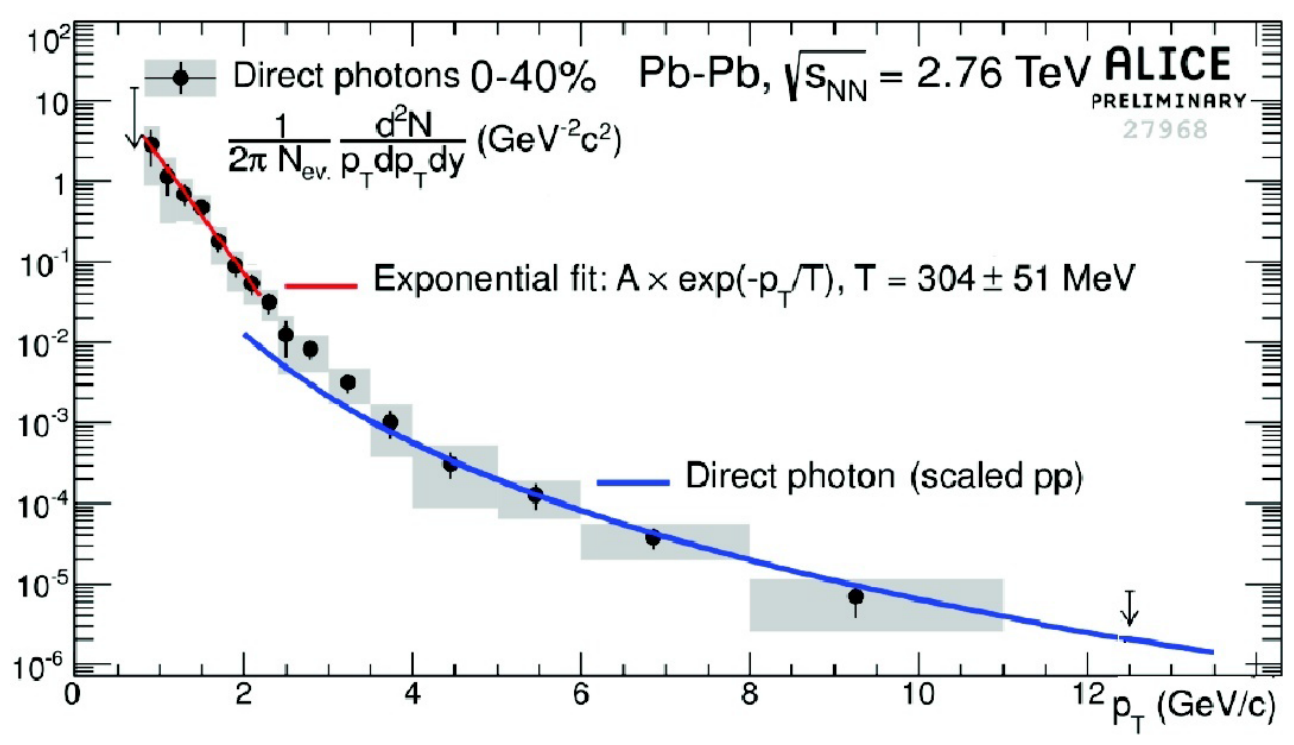

Fig. 22. Direct photon LHC- $A A$ yield; Adapted from: F. Antinori presentation July 2014.

a more democratic abundance with $u, d, s$ quarks being available in nearly equal abundance. However, this initial simple hypothesis, see ref. [15], needed to be refined with actual kinetic theory evaluation; see ref. [16], in consideration of the short time available and demonstration that quark collisions were too slow [146] to achieve this goal.

It was shown that the large abundance of strangeness depends on gluon reactions mechanism; thus the "gluon" particle component in quark gluon plasma is directly involved [145], see ref. [15]. The high strangeness density in QGP and "democratic" abundance at nearly the same level also implies that the production of (anti)baryons with multiple strangeness content is abundant, see fig. 18, which attracted experimental interest, see subsect. 4.3. The observation of strange hadrons involves the identification of non-strange hadrons and thus a full characterization of all particles emitted is possible. This in turn creates an opportunity to understand the properties of the QGP at time of hadronization, see subsect. 10.1.

\section{Hard hadrons: jet quenching}

With increasing energy, like in $p p$, also in $A A$ collisions hard parton back-scattering must occur, with a rate described by the perturbative QCD $[165,166]$. Such hard partons are observed in back-to-back jets, that is two jetlike assembly of particles into which the hard parton hadronizes. These jets are created within the primordial medium. If geometrically such a pair is produced near to the edge of colliding matter, one of the jet-partons can escape and the balancing momentum of the immersed jetparton tells us how it travels across the entire nuclear domain, in essence traversing QGP that has evolved in the collision. The energy of such a parton can be partially or completely dissipated, "thermalized" within the QGP distance traveled. Since at the production point a second high energy quark (parton) was produced, we can deduce from the "jet" asymmetry that the dense matter we form in RHI collisions is very opaque, and with some effort we can quantify the strength of such an interaction. This establishes the strength of interaction of a parton at given energy with the QGP medium.

\section{Direct photons}

Hot electromagnetic charge plasma radiates both photons and virtual photons, dileptons $[167,168]$. The hotter is the plasma, the greater is the radiation yield; thus we hope for a large early QGP stage contribution. Electromagnetic probes emerge from the reaction zone without noticeable loss. The yield is the integral over the history of QGP evolution, and the measured uncorrected yield is polluted by contributions from the ensuing hadron decays.

On the other hand, at first glance photons are the ideal probes of the primordial QGP period if one can control the background photons from the decay of strongly interacting particles such as $\pi^{0} \rightarrow \gamma \gamma$ which in general are dominant ${ }^{7}$. Recognition of the signal as direct QGP photon depends on a very precise understanding of the background.

At the highest collision energy the initial QGP temperature increases and thus direct photons should be more abundant. In fig. 22 we see the first still at the time of writing preliminary result from the Alice experiment at LHC. The yield shown is "direct"; that is, after the indirect photon part has been removed. The removal procedure appears reliable as for large $p_{\perp}$ scaled $p p$ yields match the outcome. At small $p_{\perp}$, we see a very strong excess above the scaled $p p$ yields. The $p_{\perp}$ is high enough to believe that the origin are direct $\mathrm{QGP}$ photons, and not collective charge acceleration-radiation phenomena.

\footnotetext{
7 Note that $\gamma$ when used as a symbol for photons is not to be confounded with parallel use of $\gamma$ as a fugacity, meaning is always clear in the context.
} 
A virtual photon with $q^{2} \neq 0$ is upon materialization a dilepton $e^{+} e^{-}, \mu^{+} \mu^{-}$in the final state. The dilepton yields, compared to photons, are about a factor 1000 smaller; this creates measurement challenges e.g. for large $p_{\perp}$. Backgrounds from vector meson intermediate states and decays are very large and difficult to control. Despite many efforts to improve detection capabilities and the understanding of the background, this author considers the situation as fluid and inconclusive: dilepton radiance not directly attributed to hadrons is often reported and even more often challenged. An observer view is presented in ref. [169].

\section{$J / \Psi(c \bar{c})$ yield modification}

This is the other cornerstone observable often quoted in the context of the early QGP search. The interest in the bound states of heavy charm quarks $c \bar{c}$ and in particu$\operatorname{lar} J / \Psi$ is due to their yield evolution in the deconfined state as first proposed by Matsui and Satz [170] just when first result $J / \Psi$ became available. Given that the variations in yield are subtle, and that there are many model interpretations of the effects based on different views of interaction of $J / \Psi$ in the dense matter - both confined and deconfined - this has been for a long time a livid topic which is beyond the scope of this review [171].

Modern theory addresses both "melting" and recombination in QGP as processes that modify the final $J / \Psi$ yield $[102,172]$. Recent results of the Alice collaboration [173] support, in my opinion, the notion of recombinant $c \bar{c}$ formation. Some features of these results allow suggesting that a yield equilibrium between melting and recombination has been reached for more central collisions. This is clearly a research topic, not yet suitable for a review analysis.

\section{Particle correlations and HBT}

Measurement of two particle and in particular two pion and two kaon correlations allows within the framework of geometric source interpretation the exploration of the three dimensional source size and the emission lifespan of the fireball. For a recent review and update of PHENIXRHIC results see ref. [174] and for ALICE-LHC see ref. [175]. These reports are the basis for our tacit assumption that soft hadrons emerge from the hadronization fireball with transverse size as large as $R \simeq 9 \mathrm{fm}$ for most central collisions. Aside of two particle correlation, more complex multi-particle correlations can be and are explored - their non-vanishing strength reminds us that the QGP source can have color-charge confinement related multiparticle effects that remain difficult to quantify. As an example of recent work on long range rapidity correlations see ref. [176].

\section{Fluctuations}

Any physical system that at first sight appears homogeneous will under a magnifying glass show large fluctua- tions; the color of the sky and for that matter of our planet originate in how the atmospheric density fluctuations scatter light. To see QGP fluctuation effects we need to study each individual event forming QGP apart from another. The SHARE suite of SHM programs also computes statistical particle yield fluctuations, see subsect. 9.4. The search is for large, non-statistical fluctuations that would signal competition between two different phases of matter, a phase transformation. This topic is attracting attention [177]. To see the phase transformation in action smaller reaction systems may provide more opportunity.

\subsection{Survey of LHC-ion program July 2015}

The Large Hadron Collider (LHC) in years of operation sets aside 4 weeks of run time a year to the heavy ion beam experiments, typically $A A(\mathrm{~Pb}-\mathrm{Pb})$ collisions but also $p$ $\mathrm{Pb}$. The $p p$ collision LHC run which lasts considerably longer addresses Higgs physics and beyond the standard model searches for new physics. This long run provides heavy ion experimental groups an excellent opportunity to obtain relevant data from the smallest collision system, creating a precise baseline against which $A A$ is evaluated. Furthermore, at the LHC energy, one can hope that in some measurable fraction of events conditions for QGP could be met in select, triggered events (i.e. collision class feature selected).

When LHC reaches energy of $7 \mathrm{TeV}+7 \mathrm{TeV}$ for protons, for $\mathrm{Pb}-\mathrm{Pb}$ collisions this magnet setting will correspond to a center-of-mass energy of up to $\sqrt{s_{\mathrm{NN}}}=$ $5.52 \mathrm{TeV}$ per nucleon pair in $\mathrm{Pb}-\mathrm{Pb}$ collisions. However, due to magnet training considerations the scheduled heavy ion run starting in mid-November 2015 should be at $\sqrt{s_{\mathrm{NN}}}=5.125 \mathrm{TeV}$ and the maximum energy achieved in the following year. The results we discuss in this review, see sect. 10, were obtained at a lower magnet setting in the LHC run 1, corresponding to $\sqrt{s_{\mathrm{NN}}}=2.76 \mathrm{TeV}$.

Several experiments at LHC take AA collision data.

1) The ALICE (A Large Ion Collider Experiment) was conceived specifically for the exploration of the QGP formed in nucleus-nucleus collisions at the LHC. Within the central rapidity domain $0.5 \leq y \leq 0.5$, ALICE detectors are capable of precise tracking and identifying particles over a large range of momentum. This permits the study of the production of strangeness, charm and resonances, but also multi-particle correlations, such as HBT and (moderate energy) jets. In addition, ALICE consists of a muon spectrometer allowing us to study at forward rapidities heavy-flavor and quarkonium production. The detector system also has the ability to trigger on different aspects of collisions, to select events on-line based on the particle multiplicity, or the presence of rare probes such as (di-)muons, and the electromagnetic energy from high-momentum electrons, photons and jets.

2) ATLAS (A Toroidal LHC ApparatuS) has made its name by being first to see jet quenching. It has high $p_{\perp}$ particle ID allowing the measurement of particle 

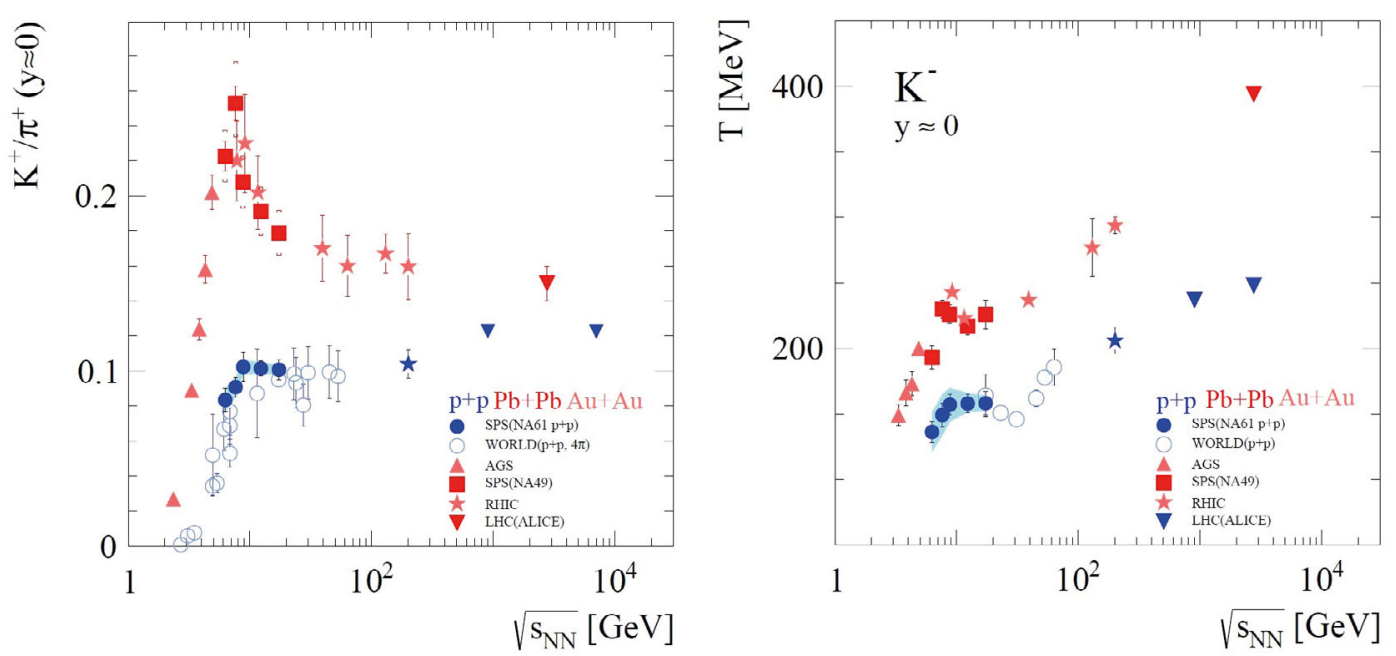

Fig. 23. The so-called horn (left) and step (right) structures in energy dependence of the $\mathrm{K}^{+} / \pi^{+}$ratio, and the inverse slope parameter of $\mathrm{K}^{-} m_{\perp}$ spectra, respectively. signal indicating threshold in strangeness to entropy yield in central $\mathrm{Pb}+\mathrm{Pb}(\mathrm{Au}+\mathrm{Au})$ collisions, from [182].

spectra in a domain inaccessible to other LHC experiments.

3) The CMS (Compact Muon Solenoid) offers high rate and high resolution calorimetry, charged particle tracking and muon identification over a wide acceptance, allowing detailed measurements of jets as well as heavyquark open and bound states. The large solid angle coverage also provides unique opportunities in the study of global observables.

The $\mathrm{LHCb}$ experiment has at present no footprint in the study of $A A$ collisions but has taken data in $p A$ trial run.

\subsection{Energy and A scan}

The smaller the size of colliding nuclei, the shorter is the collision time. Thus in collisions of small sized objects such as $p p$ or light nuclei, one cannot presume, especially at a relatively low collision energy, that primordial and yet not well understood processes (compare subsect. 5.2) will have time to generate the large amount of entropy leading to QGP formation that would allow a statistical model to work well, and in particular would allow QGP formation. This then suggests that one should explore dependence on reaction volume size, both in terms of collision centrality and a scan of projectile ion $A$.

An important additional observation is that particle production processes are more effective with increasing collision energy. Therefore the chemical equilibration is achieved more rapidly at higher energy. It seems that just about everyone agrees to this even though one can easily argue the opposite, that more time is available at lower energy. In any case, this urban legend that energy and time grow together is the main reason why QGP search experiments started at the highest available accelerator energy. This said, the question about the threshold of QGP production as a function of energy is open.
Considered from a theoretical perspective one recognizes in an energy and $A$ scan the opportunity to explore qualitative features of the QCD phase diagram in the $T$, $\mu_{\mathrm{B}}$ plane. Of particular importance is the finding of the critical point where at a finite value of $\mu_{\mathrm{B}}$ the smooth transformation between quark-hadron phases turns into an expected 1st order transition, see ref. [179]. There are other structure features of quark matter that may become accessible, for a review see ref. [40] and comments at the end of subsect. 2.2.

At CERN the multipurpose NA61 experiment surveys in its heavy-ion program tasks the domain in energy and collision system volume where threshold of deconfinement is suspected in consideration of available data. This experiment responds to the results of a study of head-on $\mathrm{Pb}-\mathrm{Pb}$ collisions as a function of energy at SPS did produce by 2010 tantalizing hints of an energy threshold to new phenomena [180-183].

There are significant discontinuities as a function of collision energy in the $\mathrm{K}^{+} / \pi^{+}$particle yield ratio, see fig. 23 on left. Similarly, the inverse slope parameter of the $m_{\perp}$ spectra of $\mathrm{K}^{-}$, see fig. 23 on right, also displays a local maximum near to $30 \mathrm{AGeV}$, that is at $3.8+3.8 \mathrm{GeV}$, $\sqrt{s_{\mathrm{NN}}}=7.6 \mathrm{GeV}$ collider energy collisions in both quantities. These behavior "thresholds" are to some degree mirrored in the much smaller $p p$ reaction system also shown in fig. 23. These remarkable results are interpreted as the onset of deconfinement as a function of collision energy.

Turning to comparable efforts at RHIC: in 2010 and 2011, RHIC ran the first phase of a beam energy scan program (RHIC-BES) to probe the nature of the phase boundary between hadrons and QGP as a function of $\mu_{\mathrm{B}}$. With beam energy settings $\sqrt{s_{\mathrm{NN}}}=7.7,11.5,19.6$, $27,39 \mathrm{GeV}$, with $14.5 \mathrm{GeV}$ included in year 2014 , complementing the full energy of $200 \mathrm{GeV}$, and the run at $62.4 \mathrm{GeV}$, a relatively wide domain of $\mu_{\mathrm{B}}$ can be probed, as the matter $v s$. anti-matter excess increases when energy decreases. For a report on these result see refs. [184,185]. 
Among the first phase of the beam energy scan discoveries is the $\mu_{\mathrm{B}}$ dependence of azimuthal asymmetry of flow of matter, $v_{2}$. Particle yield ratio fluctuations show significant deviation from Poisson expectation within HRG model. This and other results make it plausible that QGP is formed down to the lowest RHIC beam energy of $\sqrt{s_{\mathrm{NN}}}=7.7 \mathrm{GeV}$, corresponding to fixed target collision experiments at $32 \mathrm{AGeV}$. This is the collision energy where SPS energy scan also found behavior characteristic of QGP, see fig. 23. These interesting results motivate the second RHIC-BES phase after detector upgrades are completed in 2018/19.

\section{What are the conceptual challenges of the $\mathrm{QGP} / \mathrm{RHI}$ collisions program?}

In subsect. 1.1 we have briefly addressed the Why? of the RHI collision research program. Here we return to explore some of the points raised, presenting a highly subjective view of foundational opportunities that await us.

\subsection{The origin of mass of matter}

Confining quarks to a domain in space means that the typical energy each of the light quarks will have inside a hadron is $E_{q} \propto 1 / R \gg m_{q}$, where $R$ is the size of the "hole" in the vacuum - a vacuole. Imposing a sharp boundary and forbidding a quark-leak results in a squarewell-like relativistic Dirac quantum waves. This model allows quantification of $E_{q}$. One further argues that the size $R$ of the vacuole arises from the internal Fermi and Casimir pressures balancing the outside vacuum which presses to erase any vacuole comprising energy density that is higher.

In a nutshell this is the math known from within the context of quark-bag model [96-98], rounded off allowing color-magnetic hyperfine structure splitting. This model explains how baryons and mesons have a mass much greater than the sum of quark masses. It is also easy to see that a larger vacuole with hot quarks and gluons would provide a good starting point to develop a dynamical model of expanding QGP fireball formed in RHI collisions.

The advent of lattice-QCD means we can address static time independent properties of strongly interacting particles. A test of bag models ideas is the computation of the hadron mass spectrum and demonstration that the mass of hadrons is not determined by the mass of quarks bound inside. Indeed, this has been shown $[186,187]$; the confining vacuum structure contributes as much as $96 \%$ of the mass of the matter, the Higgs field the remaining few- $\%$.

Based on both bag model consideration and latticeQCD we conclude that the quantum zero-point energy of the localized, confined, light quarks governs the mass of matter. The ultimate word is, however, expected from an experiment. Most think that setting quarks free in a large vacuole created in RHI collision laboratory experiments is offering a decisive opportunity to test this understanding of mass of matter. The same lattice-QCD that provided the numerical evaluation of mass of matter, provides properties of the hot QGP Universe.

Others go even further to argue nothing needs to be confirmed: given the QCD action, the computer provides hadron spectrum and other static properties of hadron structure. For a recent review of "Lattice results concerning low-energy particle physics," see ref. [188]. That is true: the relatively good agreement of lattice-QCD theory with low-energy particle physics proves that QCD is the theory of strong interactions. In fact, many textbooks argue that this has already been settled 20 years ago in accelerator experiments, so a counter question could be, why bother to do lattice-QCD to prove QCD? One can present as example of a new insight the argument that the mass of matter is not due to the Higgs field $[186,187]$.

However, the mass argument is not entirely complete. The vacuole size $R$ directly relates to QCD vacuum properties - in bag models we relate it to the bag constant $\mathcal{B}$ describing the vacuum pressure acting on the vacuole. But is this hadron energy scale $\mathcal{B}^{1 / 4} \simeq 170 \mathrm{MeV}$ fundamental? The understanding of the scale of the QCD vacuum structure has not been part of the present-day lattice-QCD. In lattice-QCD work one borrows the energy scale from an observable. In my opinion hadron vacuum scale is due to the vacuum Higgs field, and thus the scale of hadron masses is after all due to Higgs field; it is just that the mechanism is not acting directly.

Let me explain this point of view: By the way of top interaction with Higgs there is a relation of the Higgs with the QCD vacuum scale.

a) The intersection between QCD and the Higgs field is provided by the top quark, given the remarkable value of the minimal coupling $g_{t}$

$$
g_{t} \equiv \frac{m_{t}}{\langle h\rangle} \simeq 1, \quad \alpha_{h}^{t} \equiv \frac{g_{t}^{2}}{4 \pi}=0.08 \simeq \alpha_{s}\left(m_{t}\right)=0.1 .
$$

Note that the same strength of interaction: top with gluons $\alpha_{s}\left(m_{t}\right)$, and with Higgs field fluctuations $\alpha_{h}^{t}$.

b) The size of QCD vacuum fluctuations has been estimated at $0.3 \mathrm{fm}$ [189]. This is large compared to the top quark Compton wavelength $\lambda_{t}=\hbar c / m_{t}=1.13 \times 10^{-3} \mathrm{fm}$. This means that for the top-field the $\mathrm{QCD}$ vacuum looks like a quasi-static mountainous random field driving large top-field fluctuations in the QCD vacuum.

The possible relation of the $\mathrm{QCD}$ vacuum structure via top quark with Higgs requires much more study, I hope that this will keep some of us busy in coming years.

That something still needs improvement in our understanding of strong interactions is in fact clear: Why i) all hadrons we know have $q q q$ and $q \bar{q}$ structure states, and why ii) we do not observe internal excitations of quarks in bags appearing as hadron resonances. These two questions show that how we interpret QCD within the bag model is incomplete.

I hope to have dented somewhat the belief that latticeQCD is capable of replacing the experimental study of vac- 
uum structure. In a nutshell, lattice neither explains scales of vacuum structure, nor can it address any dynamical phenomena, by necessity present in any laboratory recreation of the early Universe QGP conditions. Finally, the QCD vacuum structure paradigm needs an experimental confirmation.

\subsection{The quantum vacuum: Einstein's æther}

The quantum vacuum state determines the prevailing form of the "fundamental" physics laws. Within the standard model, the nature of particles and their interactions is determined by the transport properties of the vacuum state. As just discussed above, the mass of matter is inherent in the scale of QCD, which itself relates in a way to be studied in the future with the Higgs vacuum structure.

The existence of a structured quantum vacuum as the carrier of the laws of physics was anticipated by Lorentz, and Einstein went further seeking to reconcile this with the principles of relativity. What we call quantum vacuum, they called æether. The concluding paragraph from a lecture by Albert Einstein is creating the philosophical foundation of the quantum vacuum as carrier of laws of physics (translation by author) [190]

... space is endowed with physical qualities; in this sense the æether exists. According to the general theory of relativity, space without æether is unthinkable: without æther light could not only not propagate, but also there could be no measuring rods and clocks, resulting in non-existence of space-time distance as a physical concept. On the other hand, this æether cannot be thought to possess properties characteristic of ponderable matter, such as having parts trackable in time. Motion cannot be inherent to the æther.

A few months earlier, in November, 1919 Einstein announced the contents of this address in a letter to Lorentz: It would have been more correct if I had limited myself, in my earlier publications, to emphasizing only the nonexistence of an ather velocity, instead of arguing the total non-existence of the ather...

\subsection{The quantum vacuum: Natural constants}

In the quark-gluon plasma state of matter, we fuse and dissolve nucleons in the primordial æether state, different in its structure and properties from the æther of our experience. In Einstein's writings quoted above the case of transition between two coexistent æether states was not foreseen, but properties such as the velocity of light were seen as being defined by the æther. One should thus ask: Is velocity of light the same out there (vacuole) as it is around here? Such a question seems on first sight empty as the velocity of light connects the definition of a unit of length with the definition of a time increment. However, if $\bar{c}$ in the vacuole is the same as $c$, it means that time "advances" at the same rate there as it does here. This assumption is not necessary.

Is it possible, both in practical and in principle terms, using RHI collisions to answer if $\bar{c}=c$, where the bar indicates the property in the vacuole?

We can for example study the relation between energy and momentum of photons produced in QGP, and the rate at which these processes occur. The photon emitted is defined by its wavelength $k=1 / \lambda$, the energy of the photon is $\hbar c k$. This energy is different in the vacuole from what we observe in the laboratory - energy conservation for the photon is not maintained since the translation symmetry in time would need to be violated to make time tick differently in different vacuum states. However, global energy conservation is assured. Transition radiation, Cherenkov radiation are more mundane examples of what happens when a superluminal photon enters a dielectric medium. Thus we will need to differentiate with what would be called medium effect when considering photon propagation across the $\bar{c} \neq c$. boundary. That may be difficult.

Turning now to the rate of photon production in the vacuole: we keep to gauge invariance, thus charge cannot change between two quantum vacuum states. The way the change from the vacuole to the normal vacuum rate will be looked at is that we assume the space size of the vacuole to be measured in units of length evaluated in the normal vacuum. The rate of an electromagnetic process in modified vacuum should be, according to the Fermi golden rule proportional to $\bar{\hbar} \bar{\alpha}^{2}=e^{4} \bar{\hbar} /\left(\bar{\hbar}^{2} \bar{c}^{2}\right)$. This expression reminds that we also can have $\bar{\hbar} \neq \hbar$, but the result will involve the product $c h$ only. The rate per unit volume and time of an electromagnetic process is in the vacuole with $\Delta \bar{t}=\Delta L / \bar{c}$ is

$$
\overline{\mathcal{W}} \propto \bar{\hbar} \frac{\bar{\alpha}^{2}}{\Delta^{3} L \Delta \bar{t}} \propto \frac{1}{\bar{\hbar} \bar{c}} \frac{1}{\Delta^{4} L} .
$$

The number of events we observe is $\Delta L^{4} \overline{\mathcal{W}}$. The production of direct dileptons and direct photons is thus predicted to scale with $(\bar{\hbar} \bar{c})^{-1}$ in a space-time volume determined in our vacuum by for example the HBT method.

The above consideration cannot be applied to strong interactions since there is no meaning to $\alpha_{s}$ in the normal vacuum; we always measure $\bar{\alpha}_{s}$. Similarly, the thermal properties of the vacuole, in particular addressing the quark energy, are intrinsic properties. The direct connection of intrinsic to external properties occurs by electromagnetic phenomena. The practical problem in using the rate of electromagnetic processes to compare in-out $(\hbar c)^{-1}$ is that all production processes depend on scattering of electrically charged quanta (quarks) in QGP, and that in turn depends on a high power of $T$. This means that small changes in $\hbar c$ could be undetectable. However, it will be quite difficult to reconcile an order of magnitude $\hbar c$ modification by pushing $T$ and HBT sizes. We hope to see such studies in the near future, where one tries to determine for electromagnetic processes an in-medium strength of $\alpha$ as this is how one would reinterpret vacuole modified physical natural constants. 


\subsection{The primordial quark universe in laboratory}

Relativistic heavy ion (RHI) collisions recreate the extreme temperature conditions prevailing in the early Universe: a) dominated by QGP; b) in the era of evolution beginning at a few $\mu$ s after the big-bang; c) lasting through the time when QGP froze into individual hadrons at about 20-30 $\mu$ s. We record especially at the LHC experiments the initial matter-antimatter symmetry a nearly net-baryonfree $(B=b-\bar{b} \rightarrow 0)$ primordial QGP ${ }^{8}$. The early Universe (but not the lab experiment) evolved through the matterantimatter annihilation leaving behind the tiny $10^{-9}$ residual matter asymmetry fraction.

The question in which era the present day net baryon number of the Universe originates remains unresolved. Most believe that the net baryon asymmetry is not due to an initial condition. For baryon number to appear in the Universe the three Sakharov conditions have to be fulfilled:

1) In terms of its evolution, the Universe cannot be in the full equilibrium stage; or else whatever created the asymmetry will also undo it. This requirement is generally understood to mean that the asymmetry has to originate in the period of a phase transformation, and the focus of attention has been on electro-weak symmetry restoring condition at a temperature scale $1000 \times T_{\mathrm{H}}$. However the time available for the asymmetry to arise is in this condition on the scale of $10^{-8} \mathrm{~s}$ and not $10^{-5} \mathrm{~s}$ or longer if the asymmetry is related to QGP evolution, hadronization, and/or matter-antimatter annihilation period.

2) During this period interactions must be able to differentiate between matter and antimatter, or else how could the residual asymmetry be matter dominated? This asymmetry requires $\mathrm{CP}$-non-conservation, well known to be inherent in the SM as a complex phase of the Kobayashi-Maskawa flavor mixing.

3) If true global excess of baryons over antibaryons is to arise there must be a baryon number conservation violating process. This seems to be a requirement on fundamental interactions which constrains most when and how one must look for the asymmetry formation. It would be hard to place this in the domain of physics today accessible to experiments as no such effect has come on the horizon.

A variant model of asymmetry could be a primordial acoustical chemical potential wave inducing an asymmetry in the local distribution of quantum numbers. It has been established that at the QGP hadronization $T=T_{\mathrm{H}}$ temperature a chemical potential amplitude at the level of $0.3 \mathrm{eV}$ achieves the present day baryon to photon number in our domain of the Universe [191]. Constrained by local, electrical charge neutrality, and $B=L$ (local net baryon density equal to local net lepton density), this chemical potential amplitude is about $10^{-9}$ fraction of $T_{\mathrm{H}}$.

This insight sets the scale of energy we are looking for: the absence in the SM of any force related to baryon number and operating at the scale of $\mathrm{eV}$ is what allows us

\footnotetext{
8 Here $b, \bar{b}$ denotes baryons and antibaryons, not bottom quarks.
}

to imagine local baryon number chemical fluctuation. This "random fluctuation" resolution of the baryon asymmetry riddle implies that our matter domain in the Universe borders on an antimatter domain - however a chemical potential wave means that this boundary is where $\mu=0$ and thus where no asymmetry is present; today presumably a space domain void of any matter or antimatter. Therefore, a change from matter to antimatter across the boundary is impossible to detect by astronomical observations - we have to look for antimatter dust straying into local particle detectors. One of the declared objectives of the Alpha Magnetic Spectrometer (AMS) experiment mounted on the International Space Station (ISS) is the search for antihelium, which is considered a characteristic signature of antimatter lurking in space [192].

We recall that the acoustical density oscillation of matter is one of the results of the precision microwave background studies which explore the conditions in the Universe at temperatures near the scale of $T=0.25 \mathrm{eV}$ where hydrogen recombines and photons begin to free-steam. This is the begin of observational cosmology era. Another factor 30000 into the primordial depth of the Universe expansion, we reach the big-bang nuclear synthesis stage occurring at the scale of $T \simeq 10 \mathrm{keV}$. Abundance of helium compared to hydrogen constrains significantly the timescale of the Universe expansion and hence the present day photon to baryon ratio. A further factor 30000 increase of temperature is needed to reach the stage at which the hadronization of quark Universe occurs at Hagedorn temperature $T_{\mathrm{H}}$.

We have focused here on conservation, or not, of baryon number in the Universe. But another topic of current interest is if the hot QGP fireball in its visible energy component conserves energy; the blunt question to ask is: What if the QGP radiates darkness, that is something we cannot see? [193]. I will return under separate cover to discuss the QGP in the early Universe, connecting these different stages. For a preliminary report see ref. [194]. The understanding of the quark Universe deepens profoundly the reach of our understanding of our place in this world.

\section{Melting hadrons}

Two paths towards the quark phase of matter started in parallel in 1964-65, when on one hand quarks were introduced triggering the first quark matter paper [117], and on another, Hagedorn recognized that the yields and spectra of hadrons were governed by new physics involving $T_{\mathrm{H}}$ and he proposed the SBM [11]. This briefly addresses the events surrounding Hagedorn discovery and the resulting modern theory of hot hadronic matter.

\subsection{The tale of distinguishable particles}

In early 1978 Rolf Hagedorn shared with me a copy of his unpublished manuscript Thermodynamics of Distinguishable Particles: A Key to High-Energy Strong Interactions?, a preprint CERN-TH-483 dated 12 October 1964. 
He said there were two copies; I was looking at one; another was in the CERN archives. A quick glance sufficed to reveal that this was, actually, the work proposing a limiting temperature and the exponential mass spectrum. Hagedorn explained that upon discussions of the contents of his paper with Léon Van Hove, he evaluated in greater detail the requirements for the hadron mass spectrum and recognized a needed fine-tuning. Hagedorn concluded that his result was therefore too arbitrary to publish, and in the CERN archives one finds Hagedorn commenting on this shortcoming of the paper, see chapt. 18 in ref. [1].

However, Hagedorn's "Distinguishable Particles" is a clear stepping stone on the road to modern understanding of strong interactions and particle production. The insights gained in this work allowed Hagedorn to rapidly invent the Statistical Bootstrap Model (SBM). The SBM paper Statistical Thermodynamics of Strong Interactions at High Energies, preprint CERN-TH-520 dated 25 January 1965 , took more than a year to appear in $\operatorname{press}^{9}$ [11].

The beginning of a new idea in physics often seems to hang on a very fine thread: was anything lost when Thermodynamics of Distinguishable Particles remained unpublished? And what would Hagedorn do after withdrawing his first limiting-temperature paper? My discussion of the matter with Hagedorn suggests that his vision at the time of how limiting temperature could be justified evolved very rapidly. Presenting his more complete insight was what interested Hagedorn and motivated his work. Therefore, he opted to work on the more complete theoretical model, and publish it, rather than to deal with complications that pressing Thermodynamics of Distinguishable Particles would generate.

While the withdrawal of the old, and the preparation of an entirely new paper seemed to be the right path to properly represent the evolving scientific understanding, today's perspective is different. In particular the insight that the appearance of a large number of different hadronic states allows to effectively side-step the quantum physics nature of particles within statistical physics became essentially invisible in the ensuing work. Few scientists realize that this is a key property in the SBM, and the fundamental cause allowing the energy content to increase without an increase in temperature.

In the SBM model, a hadron exponential mass spectrum with the required "fine-tuned" properties is a natural outcome. The absence of Hagedorn's Distinguishable Particles preprint delayed the recognition of the importance of the invention of the SBM model. The SBM paper without its prequel looked like a mathematically esoteric work; the need for exponential mass spectrum was not immediately evident.

Withdrawal of Distinguishable Particles also removed from view the fact that quantum physics in hot hadronic matter loses its relevance, as not even Boltzmann's $1 / n$ ! factor was needed, the exponential mass spectrum effectively removes it. Normally, the greater the density of particles, the greater the role of quantum physics. To

\footnotetext{
${ }^{9}$ Publication was in Nuovo Cimento Suppl. 3, 147 (1965) actually printed in April 1966.
}

the best of my knowledge the dense, strongly interacting hadronic gas is the only physical system where the opposite happens. Thus surfacing briefly in Hagedorn's withdrawn Thermodynamics of Distinguishable Particles paper, this original finding faded from view. Hagedorn presented a new idea that has set up his SBM model, and for decades this new idea remained hidden in archives.

On the other hand, the Hagedorn limiting temperature $T_{\mathrm{H}}$ got off the ground. Within a span of only 90 days between the withdrawal of his manuscript, and the date of his new CERN-TH preprint, Hagedorn formulated the SBM. Its salient feature is that the exponential mass spectrum arises from the principle that hadrons are clusters comprising lighter (already clustered) hadrons ${ }^{10}$. The key point of this second paper is a theoretical model based on the very novel idea of hadrons made of other hadrons. Such a model bypasses the need to identify constituent content of all these particles. And, Hagedorn does not need to make explicit the phenomenon of Hadron distinguishability that clearly was not easy to swallow just 30 years after quantum statistical distributions saw the light of day.

Clustering pions into new hadrons and then combining these new hadrons with pions, and with already preformed clusters, and so on, turned out to be a challenging but soluble mathematical exercise. The outcome was that the number of states of a given mass was growing exponentially. Thus, in SBM, the exponential mass spectrum required for the limiting temperature arose naturally $a b \mathrm{ini}$ tio. Furthermore the model established a relation between the limiting temperature, the exponential mass spectrum slope, and the pion mass, which provides the scale of energy in the model.

Models of the clustering type are employed in other areas of physics. An example is the use of the $\alpha$-substructure in the description of nuclei structure: atomic nuclei are made of individual nucleons, yet improvement of the understanding is achieved if we cluster 4 nucleons (two protons and two neutrons) into an $\alpha$-particle substructure.

The difference between the SBM and the nuclear $\alpha$ model is that the number of input building blocks in SBM i.e. pions and more generally of all strongly interacting clusters is not conserved but is the result of constraints such as available energy. As result one finds rapidly growing with energy size of phase space with undetermined number of particles. This in turn provides justification for the use of the grand canonical statistical methods in the description of particle physics phenomena at a time when only a few particles were observed.

\subsection{Roots and contents of the SBM}

The development of SBM in 1964/65 had a few preceding pivotal milestones, see chapt. 17 in ref. [1] for a fully referenced list. One should know seeing point 1) below

\footnotetext{
10 In this paper, as is common today, we refer to all discovered hadron resonance states - Hagedorn's clusters - as resonances, and the undiscovered "heavy" resonances are called Hagedorn states
} 
that it was Heisenberg who hired Hagedorn as a postdoc in June 1952 to work on cosmic emulsion "evaporation stars", and soon after, in 1954, sent him on to join the process of building CERN:

1) The realization and a first interpretation of many secondaries produced in a single hadron-hadron collision (Heisenberg 1936 [195]).

2) The concept of the compound nucleus and its thermal behavior (1936-1937).

3) The construction of simple statistical/thermodynamical models for particle production in analogy to compound nuclei (1948-1950) (Koppe 1949 [56,57], Fermi 1950 [58]).

Enter Hagedorn:

4) The inclusion of resonances to represent interactions recognized via phase shifts (Belenky 1956 [48]).

5) The discovery of limited $\left\langle p_{\perp}\right\rangle$ (1956).

6) The discovery that fireballs exist and that a typical $p p$ collision seems to produce just two of them, projectile and target fireball (1954-1958).

7) The discovery that large-angle elastic cross-sections decrease exponentially with CM energy (1963).

8) The discovery of the parameter-free and numerically correct description of this exponential decrease buried in Hagedorn's archived Monte Carlo phase-space results obtained earlier at CERN (1963).

Hagedorn introduced a model based on an unlimited sequence of heavier and heavier bound and resonance states he called clusters ${ }^{11}$, each being a possible constituent of a still heavier resonance, while at the same time being itself composed of lighter ones. The pion is the lightest "one-particle cluster". Hadron resonance states are due to strong interactions; if introduced as new, independent particles in a statistical model, they express the strong interactions to which they owe their existence. To account in full for strong interactions effects we need all resonances; that is, we need the complete mass spectrum $\rho(m)$.

In order to obtain the mass spectrum $\rho(m)$, we will implement in mathematical terms the self-consistent requirement that a cluster is composed of clusters. This leads to the "bootstrap condition and/or bootstrap equation" for the mass spectrum $\rho(m)$. The integral bootstrap equation $(\mathrm{BE})$ can be solved analytically with the result that the mass spectrum $\rho(m)$ has to grow exponentially. Consequently, any thermodynamics employing this mass spectrum has a singular temperature $T_{\mathrm{H}}$ generated by the asymptotic mass spectrum $\rho(m) \sim \exp \left(m / T_{0}\right)$. Today this singular temperature is interpreted as the temperature where (for baryon chemical potential $\mu_{\mathrm{B}}=0$ ) the phase conversion of hadron gas $\longleftrightarrow$ quark-gluon plasma occurs.

\subsection{Implementation of the model}

Let us look at a simple toy model proposed by Hagedorn to illustrate the Frautschi-Yellin reformulation $[12,19]$ of

\footnotetext{
11 In the older literature Hagedorn and others initially called decaying clusters fireballs, this is another example of how a physics term is recycled in a new setting.
}

the original model which we find in comparable detail in ref. [15], also shown in subsect. 2.1, eq. (4). Like in SBM, in the toy model particle clusters are composed of clusters; however we ignore kinetic energy. Thus

$$
\begin{aligned}
\rho(m)= & \delta\left(m-m_{0}\right) \\
& +\sum_{n=2}^{\infty} \frac{1}{n !} \int \delta\left(m-\sum_{i=1}^{n} m_{i}\right) \prod_{i=1}^{n} \rho\left(m_{i}\right) \mathrm{d} m_{i} .
\end{aligned}
$$

In words, the cluster with mass $m$ is either the "input particle" with mass $m_{0}$ or else it is composed of any number of clusters of any masses $m_{i}$ such that $\Sigma m_{i}=m$. We Laplace-transform eq. (22):

$$
\begin{aligned}
\int \rho(m) \mathrm{e}^{-\beta m} \mathrm{~d} m= & \mathrm{e}^{-\beta m_{0}} \\
& +\sum_{n=2}^{\infty} \frac{1}{n !} \prod_{i=1}^{n} \int \mathrm{e}^{-\beta m_{i}} \rho\left(m_{i}\right) \mathrm{d} m_{i} .
\end{aligned}
$$

Define

$$
z(\beta) \equiv \mathrm{e}^{-\beta m_{0}}, \quad G(z) \equiv \int \mathrm{e}^{-\beta m} \rho(m) \mathrm{d} m .
$$

Thus eq. (23) becomes $G(z)=z+\exp [G(z)]-G(z)-1$ or

$$
z=2 G(z)-\mathrm{e}^{G(z)}+1
$$

which provides implicitly the function $G(z)$, the Laplace transform of the mass spectrum.

A graphic solution is obtained drawing $z(G)$ in fig. 24 top frame a) and transiting in fig. 24 from top a) to bottom frame b) by exchanging the axis. The parabola-like maximum of $z(G)$ implies a square root singularity of $G(z)$ at $z_{0}$, first remarked by Nahm [27]

$z_{\max }(G) \equiv z_{0}=\ln 4-1=0.3863 \ldots, \quad G_{0}=G\left(z_{0}\right)=\ln 2$,

as also shown in fig. 24 .

It is remarkable that the "Laplace-transformed BE" eq. (25) is "universal" in the sense that it is not restricted to the above toy model, but turns out to be the same in all (non-cutoff) realistic SBM cases $[12,19]$. Moreover, it is independent of:

- the number of space-time dimensions [196]; the "toy model" extends this to the cae of "zero"-space dimensions;

- the number of "input particles" ( $z$ becomes a sum over modified Hankel functions of input masses);

- Abelian or non-Abelian symmetry constraints [197].

Upon inverse Laplace asymptotic transformation of the Bootstrap function $G(z)$ one obtains

$$
\rho(m) \sim m^{-3} \mathrm{e}^{m / T_{\mathrm{H}}}
$$

where in the present case (not universally):

$$
T_{\mathrm{H}}=-\frac{m_{0}}{\ln z_{0}}=\frac{m_{0}}{0.95} .
$$



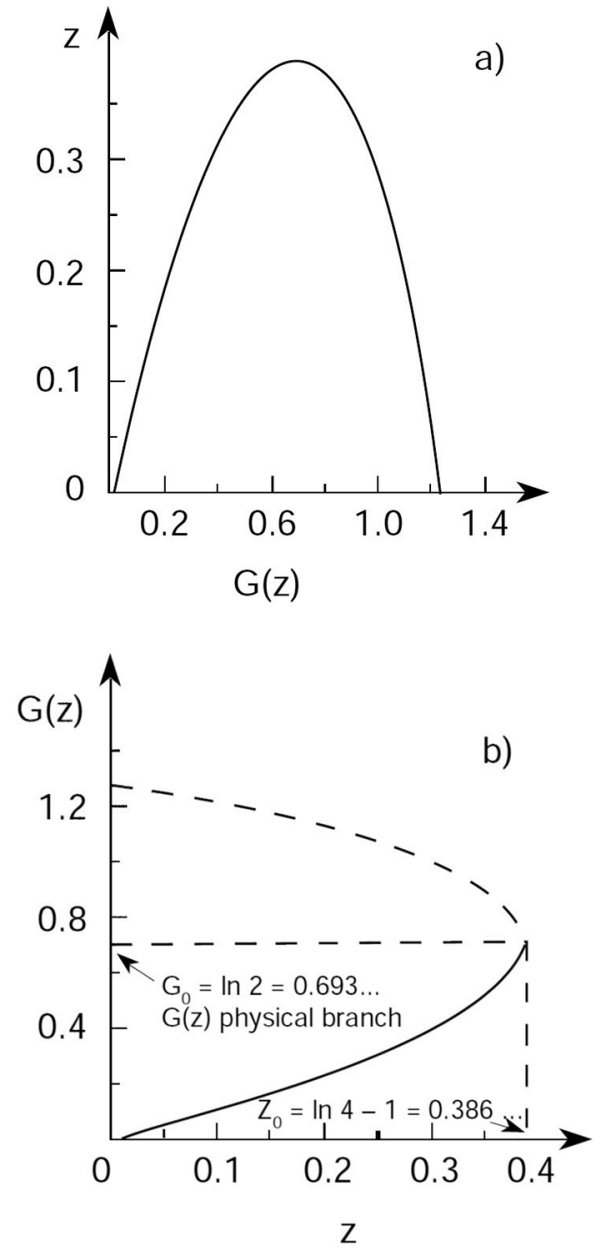

Fig. 24. (a) $z(G)$ according to eq. (25). (b) Bootstrap function $G(z)$, the graphical solution of eq. (25). The dashed line represents the unphysical branch. The root singularity is at $\varphi_{0}=\ln (4 / \mathrm{e})=0.3863$.

Using the natural choice $m_{0}=m_{\pi}$ we obtain:

$$
T_{\mathrm{H}}(\text { toy model })=145 \mathrm{MeV} \text {. }
$$

The simple toy model already yields all essential features of SBM: the exponential mass spectrum with $a=3$ and the right magnitude of $T_{\mathrm{H}}$.

\subsection{Constituents of finite size}

The original point-volume bootstrap model was adapted to be applicable to collisions of heavy ions where the reaction volume was relevant. This work began in 1977 and was in essence complete by $1980[137,28,198]$, see the details presented in ref. [15]. The new physics is that cluster volumes are introduced.

For overview of work that followed see for example ref. [199]. However, "in principle" we are today where the subject was when the initial model was completed in 1979. While many refinements were proposed, these were in physical terms of marginal impact. A new well-posed question is how the van der Waals excluded volume extension of Hagedorn SBM connects to present day latticeQCD [200], and we address this in the next subsection.

Before we discuss that, here follows one point of principle. Current work takes for granted the ability to work in a context similar to non-relativistic gas including relativistic phase space. This is not at all self-evident. To get there, see also ref. [15], we argued that particle rest-frame volumes had to be proportional to particle masses. Following Touschek [201], we defined a "four-volume"; arbitrary observer would attribute to each particle the 4-volume $V_{i}^{\mu}$ moving with particle four-velocity $u_{i}^{\mu}$

$$
V_{i}^{\mu}=V_{i} u_{i}^{\mu}, \quad u_{i}^{\mu} \equiv \frac{p_{i}^{\mu}}{m_{i}} .
$$

The entire volume of all particles is comoving with the four-velocity of the entire particle assembly of mass $m$

$$
\begin{aligned}
V^{\mu} & =V u^{\mu}, & u^{\mu} & =\frac{p^{\mu}}{m} \\
p^{\mu} & =\sum_{i=1}^{n} p_{i}^{\mu}, & m & =\sqrt{p_{\mu} p^{\mu}} .
\end{aligned}
$$

We explored a simple additive model applicable when all hadrons have the same energy density, see ref. [15]

$$
\frac{V}{m}=\frac{V_{i}}{m_{i}}=\text { const. }=4 \mathcal{B},
$$

where the proportionality constant is written $4 \mathcal{B}$ in order to emphasize the similarity to MIT bags [96-98], which have the same mass-volume relation in absence of any other energy scale. However, in QCD two relevant scales enter in higher order: that of strange quark mass, and parameters characterizing the running of QCD parameters; the coupling constant $\alpha_{s}$ and mass of strange quark $m_{s}$ are here relevant.

Given that the assembly of particles of mass $m$ occupies a comoving volume $V$ and the same applies to the constituent particles and their volumes one can henceforth ignore the Lorentz covariance challenges associated with introduction of particle proper volume. However, this has been shown only if all hadrons have the same energy density, nobody extended this argument to a more general case.

In an independent consideration the energy spectrum of such SBM clusters we obtained and that of MIT bags was found to be the same [202-204]. This suggests these two models are two different views of the same object, a snapshot taken once from the hadron side, and another time from the quark side. MIT bags "consist of" quarks and gluons, SBM clusters of hadrons. This leads on to a phase transition to connect these two aspects of the same, as is further developed in ref. [15]; the model of the phase boundary defined by the MIT bags was continued by Gorenstein and collaborations, see refs. [205,206].

\subsection{Connection with lattice-QCD}

Today the transformation between hadrons and QGP is characterized within the lattice-QCD evaluation of the 


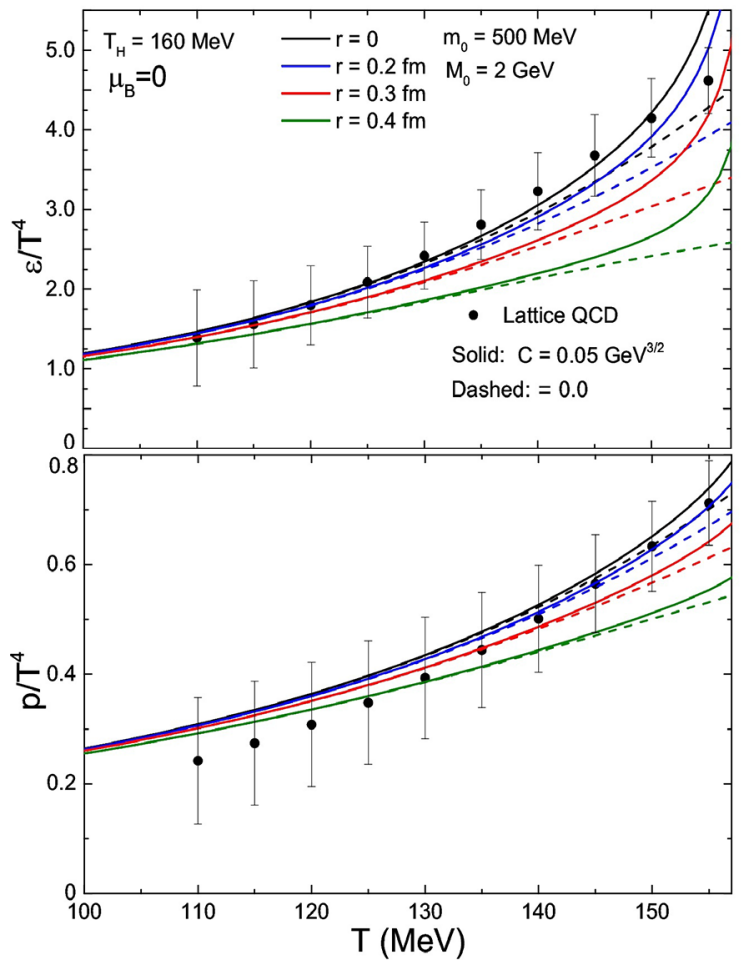

Fig. 25. Lattice-QCD [72] energy density $\varepsilon / T^{4}$ and Pressure $P / T^{4}$ for $T<155, \mu_{\mathrm{B}}=0 \mathrm{MeV}$, in RHG in a model with excluded volume parameter $r=0,0.2, .0 .3,0.4$ (dashed lines) and allowing for extension of the HRG with exponential mass spectrum (solid lines) for assumed $T_{\mathrm{H}}=160 \mathrm{MeV}$. See text. From ref. [200].

thermal properties of the quark-hadron Universe. In the context of our introductory remarks we have addressed the close relation of the HRG with the lattice results, see subsect. 2.4 and in particular fig. 6. But what does this agreement between lattice-QCD and HRG have to say about SBM of hadrons of finite size? That is an important question, it decides also the fate of the 1979 effort described in ref. [15].

Vovchenko, Anchishkin, and Gorenstein [200] analyzed the lattice-QCD for the pressure and energy density at $T<155, \mu_{\mathrm{B}}=0 \mathrm{MeV}$ within the hadron resonance gas model allowing for effects of both the excluded volume and the undiscovered part of Hagedorn mass spectrum. That work is within a specific model of finite sized hadron gas: particles occupy a volume defined by $v=16 \pi r^{3} / 3$ where $r$ is a parameter in range $0<r<0.4 \mathrm{fm}$, and it is the density of particles that characterizes the size of excluded volume.

The shape of their exponentially extended mass spectrum

$$
\rho(m)=C \frac{\theta\left(m-M_{0}\right)}{\left(m^{2}+m_{0}^{2}\right)^{a / 2}} \mathrm{e}^{m / T_{\mathrm{H}}},
$$

where the authors assumed with Hagedorn $T_{\mathrm{H}}=160 \mathrm{MeV}$, $m_{0}=0.5 \mathrm{GeV}$, and placed the cutoff at $M_{0}=2 \mathrm{GeV}$. Especially the assumed $a=5 / 2$ is in conflict with prior art, see subsect. 2.2, and the sharp cutoff leaves an unfilled "hole" in the intermediate mass domain. The authors report in a side remark that their results are insensitive to a change $a=5 / 2 \rightarrow a=3$ with appropriate other changes but this does not resolve the above sharp cutoff matter. Note that the normalization parameter $C$ in eq. (32) is the only free parameter and for $C=0$ the complement states are excluded (dashed lines in fig. 25), the model reverts to be HRG with finite size particle volume, but only for $r \neq 0$, for $r=0$ we have point HRG. How this model modifies energy density $\varepsilon / T^{4}$ and pressure $P / T^{4}$ is seen in fig. 25.

The authors conclude that lattice data exclude taking the two effects apart, i.e. consideration of each of these individually. This is so since for $C=0$ the fit of pressure fig. 6 favors finite hadron volume parameter $r \lesssim 0.4 \mathrm{fm}$; however the best fit of energy density shows $r \cong 0$. When both: excluded volume $r \neq 0$, and heavy resonances $C \neq 0$ are considered simultaneously the model works better: the effect of finite volume and the possibly yet undiscovered high mass Hagedorn mass spectrum thus complement each other when considered simultaneously: the suppression effects for pressure $P / T^{4}$ and energy density $\varepsilon / T^{4}$ due to the excluded volume effects and the enhancement due to the Hagedorn mass spectrum make the data fit marginally better as we can see in fig. 25 .

In effect ref. [200] tests in quantitative fashion the sensitivity of the lattice-QCD results to physics interpretation: it seems that even if and when the lattice results should be a factor 5 more precise, the correlation between the contribution of undiscovered states and the van der Waals effect will compensate within error margin.

As disappointing as these results may seem to some, it is a triumph for the physics developed in 1979. Namely, results of ref. [200] also mean that upon a reasonable choice of the energy density in Hadrons $4 \mathcal{B}$, the model presented in ref. [15] will fit well the present day lattice-QCD data $T<155, \mu_{\mathrm{B}}=0 \mathrm{MeV}$, since it has both the correct mass spectrum, and the correct van der Waals repulsion effects due to finite hadron size. Therefore, this model is bound to be accurate as a function of $\mu_{\mathrm{B}}$ as well.

Reading in ref. [15] it seems that the perturbative QCD phase has properties that do not match well to the SBM, requiring a strong 1st order phase transition matching to SBM. This was a result obtained with a fixed value of $\alpha_{s}$ in thermal-QCD. The results of lattice-QCD teach us that a more refined model with either running $\alpha_{s}$ and/or thermal quarks and gluon masses $[6,110]$ is needed. Contemporary investigation of the latest lattice-QCD results in such terms is promising [111] as we already mentioned in subsect. 3.4 .

\section{Hadronization of QGP fireball}

In this section the method and implementation of the fireball hadronization model will be presented allowing us to address the task defined in subsect. 5.3. This model was already described in subsect. 2.7 and thus we can proceed rapidly to develop the technical details. 


\subsection{A large parameter set}

Our task is to describe precisely a multitude of hadrons by a relatively small set of parameters. This then allows us to characterize the drop of QGP at the time of hadronization. In our view, the key objective is to characterize the source of hadrons rather than to argue about the meaning of parameter values in a religious fashion. For this procedure to succeed, it is necessary to allow for the greatest possible flexibility in the characterization of the particle phase space, consistent with conservation laws and related physical constraints at the time of QGP hadronization. For example, the number yield of strange and light quark pairs has to be nearly preserved during QGP hadronization. Such an analysis of experimental hadron yield results requires a significant bookkeeping and fitting effort, in order to allow for resonances, particle widths, full decay trees and isospin multiplet sub-states. We use SHARE (Statistical HAdronization with REsonances), a data analysis program available in three evolution stages for public use [207-209].

The important parameters of the SHM, which control the relative yields of particles, are the particle specific fugacity factors $\lambda \equiv \mathrm{e}^{\mu / T}$ and the space occupancy factors $\gamma$. The fugacity is related to particle chemical potential $\mu=T \ln \lambda . \mu$ follows a conserved quantity and senses the sign of "a charge". Thus it flips sign between particles and antiparticles.

The resultant shape of the Fermi-Dirac distribution is seen in eq. (17). The occupancy $\gamma$ is in Boltzmann approximation the ratio of produced particles to the number of particles expected in chemical equilibrium. Since there is one quark and one antiquark in each meson, yield is proportional to $\gamma_{q}^{2}$ and accordingly the baryon yield to $\gamma_{q}^{3}$. When necessary we will distinguish the flavor of the valance quark content $q=u, d, s, \ldots$

The occupancy parameters describing the abundance of valance quarks counted in hadrons emerge in a complex evolution process described in subsect. 5.3. In general, we expect a non-equilibrium value $\gamma_{i} \neq 1$. A much simplified argument to that used in subsect. 5.3 is to to assume that we have a completely equilibrated QGP with all quantum charges zero (baryon number, etc.) and thus, in QGP all $\lambda_{i}=1, \gamma_{i}=1$. Just two parameters describe the QGP under these conditions: temperature $T$ and volume $V$.

This state hadronizes preserving energy, and increasing or preserving entropy and essentially the number of pairs of strange quarks. On the hadron side temperature $T$ and volume $V$ would not suffice to satisfy these constraints, and thus we must at least introduce $\gamma_{s}>1$. The value is in general above unity because near to chemical equilibrium the QGP state contains a greater number of strange quark pairs compared to the hadron phase space.

Table 3 presents the here relevant parameters which must be input with their guessed values or assumed conditions, in order to run the SHARE with CHARM program as input in file thermo.data. When and if we allow $\gamma_{s}$ to account for excess of strangeness content, we must also introduce $\gamma_{q}$ to account for a similar excess of QGP light quark content as already discussed in depth in subsect. 5.3.
Table 3. Thermal parameters and their SHARE name. The values are to be presented in units $\mathrm{GeV}$ and $\mathrm{fm}^{3}$, where applicable.

\begin{tabular}{lll}
\hline Symbol & Parameter & Parameter description \\
\hline$V$ & norm & absolute normalization in $\mathrm{fm}^{3}$ \\
$T$ & temp & chemical freeze-out temperature $T$ \\
$\lambda_{q}$ & lamq & light quark fugacity factor \\
$\lambda_{s}$ & lams & strangeness fugacity factor \\
$\gamma_{q}$ & gamq & light quark phase space occupancy \\
$\gamma_{s}$ & gams & strangeness phase space occupancy \\
$\lambda_{3}$ & lmi3 & $I_{3}$ fugacity factor (eq. (54)) \\
$\gamma_{3}$ & gam3 & $I_{3}$ phase space occupancy (eq. $\left.(52)\right)$ \\
\hline$\lambda_{c}$ & lamc & charm fugacity factor $e . g . \lambda_{c}=1$ \\
$N_{c+\bar{c}}$ & Ncbc & number of $c+\bar{c}$ quarks \\
$T_{c} / T$ & tc2t & ratio of charm to the light quark \\
& & hadronization temperature \\
\hline
\end{tabular}

In regard to the parameters $\gamma_{q}, \gamma_{s} \neq 1$, we note that:

a) We do not know all hadronic particles, and the incomplete hadron spectrum used in SHM can be to some degree absorbed into values of $\gamma_{s}, \tilde{\gamma}_{q}$;

b) In our analysis of hadron production results we do not fit spectra but yields of particles. This is so since the dynamics of outflow of matter in an exploding fireball is hard to control; integrated spectra (i.e., yields) are not affected by collective flow of hot matter.

c) $\gamma_{q}, \gamma_{s} \neq 1$ complement $\gamma_{c}, \gamma_{b}$ to form a set of nonequilibrium parameters.

Among the arguments advanced against use of chemical non-equilibrium parameters is the urban legend that it is hard, indeed impossible, to find in the enlarged parameter space a stable fit to the hadron yield data. A large set of parameters often allows spurious local minima which cloud the physical minimum - when there are several fit minima, a random search can oscillate between such nonphysics minima rendering the fit neither reproducible, nor physically relevant.

This problem is solved as follows using the SHARE suite of programs: we recall that SHARE allows us to use any of the QGP bulk properties to constrain fits to particle yield. In extreme, one can reverse the process: given a prescribed fireball bulk properties one can fit a statistical parameter set, provided that the information that is introduced is sufficient.

To find a physics best fit, what a practitioner of SHARE will do is to loosely constrain the physical bulk properties at hadronization. One speeds up considerably the convergence by requiring that fits satisfy some ballpark value such as $\epsilon=0.45 \pm 0.15 \mathrm{GeV} / \mathrm{fm}^{3}$. Once a good physics minimum is obtained, a constraint can be removed. If the minimum is very sharp, one must repeat this process recursively; when imposing a value such as a favorite value of freeze-out $T$, the convergence improvement constraint has to be adjusted. 


\subsection{Rapidity density yields $\mathrm{dN} / \mathrm{dy}$}

In fitting the particle produced at RHIC and LHC energies we rarely have full information available about the yields. The detectors are typically designed to either cover the center of momentum domain (central rapidity) or the forward/backward "projectile/target" domains. Thus practically always - with the exception of results in SPS range of energies - we do need to focus our analysis on particle yields emerging from a domain, typically characterized by rapidity $y$ of a particle.

As a reminder, the rapidity of a particle $y$ replaces in set of kinematic variables the momentum component parallel to the axis RHI motion. For a particle of mass $m$ with momentum vector $\boldsymbol{p}=\boldsymbol{p}_{\|}+\boldsymbol{p}_{\perp}$ split into components parallel and perpendicular to the axis RHI motion, the relation is

$$
p_{\|}=E_{\perp} \sinh y, \quad E_{\perp}=\sqrt{m^{2}+p_{\perp}^{2}},
$$

which implies the useful relation $E=E_{\perp} \cosh y$.

Rapidity is popular due to the additivity of the value of $y$ under a change of reference frame in the $\|$-direction characterized by the Lorentz transformation where $\cosh y_{\mathrm{L}}=$ $\gamma_{\mathrm{L}}, \sinh _{\mathrm{L}}=\beta_{\mathrm{L}} \gamma_{\mathrm{L}}$. In this restricted sense rapidity replaces velocity in the context of relativistic motion. The value of $y$ is recognized realizing that a fireball emitting particles will have some specific value of $y_{\mathrm{f}}$ which we recognize displaying particle yields as function of rapidity, integrated with respect to $\boldsymbol{p}_{\perp}$.

The meaning of an analysis of particle data multiplicities $\mathrm{d} N / \mathrm{d} y_{p}$ is that we look at the particles that emerged from $\mathrm{d} V / \mathrm{d} y_{p}$ : in the fireball incremental volume $\mathrm{d} V$ per unit of rapidity of emitted particles $\mathrm{d} y_{p}$,

$$
\frac{\mathrm{d} N}{\mathrm{~d} y} \propto \frac{\mathrm{d} V}{\mathrm{~d} y} \equiv D_{\perp}(L) \frac{\mathrm{d} L}{\mathrm{~d} y}
$$

where $D_{\perp}$ is the transverse surface at hadronization of the fireball. Considering the case of sufficiently high energy where one expects that particle yields $\mathrm{d} N / \mathrm{d} y_{p}$ are flat as function of rapidity, we can expect that $D_{\perp}(L) \simeq D_{\perp}(L=$ 0 ) and thus $\mathrm{d} L / \mathrm{d} y_{p}=$ const., where $L=0$ corresponds to the CM-location of the hadron-hadron collision.

The quantity $\mathrm{d} L / \mathrm{d} y_{p}$ relates to the dynamics of each of the positions $L$ from which measured particles emerge with a measured rapidity $y_{p}$. Each such location has its proper time $\tau$ which applies to both the dynamics of the longitudinal volume element $\mathrm{d} L$ and the dynamics of particle production from this volume element. We thus can write

$$
\frac{\mathrm{d} L}{\mathrm{~d} y_{p}} \equiv \frac{\mathrm{d} L / \mathrm{d} \tau}{\mathrm{d} y_{p} / \mathrm{d} \tau}=\frac{f\left(y_{L}\right)}{\mathrm{d}\left(y_{\mathrm{th}}+y_{L}\right) / \mathrm{d} \tau}=\text { const. }
$$

In the last step we recognize the longitudinal dynamics introducing the local flow rapidity $y_{L}$ in the numerator where $\mathrm{d} L / \mathrm{d} \tau=f\left(y_{L}\right)$, and in the denominator given the additivity of rapidity we can break up the particle rapidity

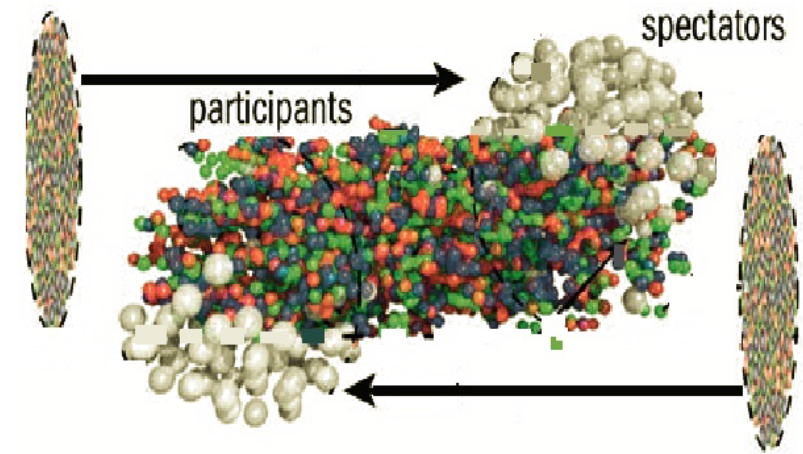

Fig. 26. Illustration of relativistic heavy ion collision: two Lorentz-contracted nuclei impact with offset, with some of the nucleons participating, and some remaining spectators, i.e. nucleons that miss the other nucleus, based on ref. [210].

into the longitudinal dynamics $y_{L}$ and the thermal component $y_{\text {th }}$, describing the statistical thermal production of particles. We have so obtained

$$
f\left(y_{L}\right)=R\left(\frac{\mathrm{d} y_{\mathrm{th}}}{\mathrm{d} \tau}+\frac{\mathrm{d} y_{L}}{\mathrm{~d} \tau}\right) .
$$

Since we form $\mathrm{d} N / \mathrm{d} y$ observing many particles emitted forward $\left(y_{\mathrm{th}}>0\right)$ or backward $\left(y_{\mathrm{th}}<0\right)$ in rapidity with respect to local rest frame, the statistical term averages out and thus we obtain as the requirement for a flat $\mathrm{d} N / \mathrm{d} y$ that the local longitudinal flow satisfies

$$
\frac{\mathrm{d} L}{\mathrm{~d} \tau}=f\left(y_{L}\right)=R \frac{\mathrm{d} y_{L}}{\mathrm{~d} \tau},
$$

that is a linear relation

$$
L-L_{0}=R\left(y_{L}-y_{0}\right) .
$$

It is tempting to view $f\left(y_{L}\right) \equiv \mathrm{d} L / \mathrm{d} \tau=\sinh y_{L}$ as we would expect if $L$ were a coordinate of a material particle. The implicit system of equations allows us then to determine the dependence of $y_{L}$ and thus $L$ on $\tau$ and thus of time evolution in eq. (34) and the relation of $\mathrm{d} V / \mathrm{d} y$ with geometric (HBT) volume, a connection that is at present not understood. This will be a topic for further study.

\subsection{Centrality classes}

When two atomic nuclei collide at relativistic speed, only matter in the collision path, see fig. 26, participates in the reaction. Two fraction of nuclei are shaved of and fly by along collisions axis - we call these nucleons spectators.

The sum of the number of participants and spectators must be exactly the number of nucleons introduced into the reaction: for $\mathrm{Pb}-\mathrm{Pb}$ this number is $2 A=416$ or perhaps better said, there are $N_{q}=1248$ valance $u, d$-quarks, and for $\mathrm{Au}-\mathrm{Au}$ we have $2 A=358$ or $N_{q}=1074$. How many of these quarks actually have interacted in each reaction is hard to know or directly measure. One applies a "trigger" to accept a class of collision events which then is characterized in terms of some macroscopic observable relating 


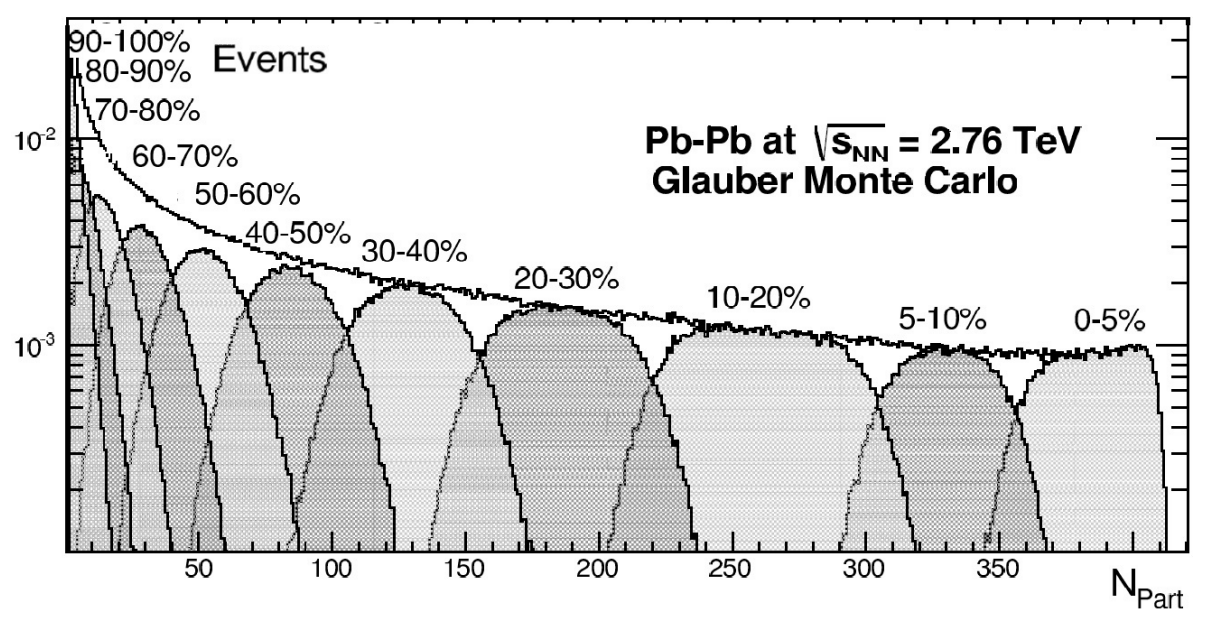

Fig. 27. Distribution in $N_{\text {part }}$ for each of experimental trigger classes called $a-b \%$ based on a MC Glauber model, data from ref. [211].

to a nearly forward flying spectator. A numerical model connects the artificially created reaction classes with the mean number of participants $N_{\text {part }}$ that contributed. For further details for the LHC work we refer to the recent ALICE review of their approach [211].

In fig. 27 we see how this works. All inelastic collision classes are divided into groups related to how big a fraction of all inelastic events the trigger selects. So $0-5 \%$ means that we are addressing the $5 \%$ most central collisions, nearly head-on. How head-on this is we can see by considering the distribution in $N_{\text {part }}$ one obtains in the Monte-Carlo Glauber model as shown in fig. 27.

How do we know that such classification, that is a characterization of events in terms of some forward observable which is model-converted into participant distribution, is meaningful? Experimental work provides direct confirmation by connecting different observables [211]. I will in the analysis of other experimental results evaluate specific properties of the fireball of matter in terms of the number of participants. Some of these properties turn out to be very flat across many of the collision classes as a function of $N_{\text {part }}$ which entered into the discussion. This shows that the expected extensivity of the property holds: as more participants participate the system expands accordingly. Moreover, this finding also validates the analysis method, a point which will be raised in due time.

\subsection{Particle yields and fluctuations}

For full and correct evaluation of the final hadron state in the LHC era, one has to calculate:

1) primary particle yields at chemical freeze-out;

2) charm hadron decays for a given charm quark abundance, followed by

3) decays of all hadron resonances.

The point 2. is the new module that rounds of SHARE for LHC energies [209].

Every hadron of species $i$ with energy $E_{i}=\sqrt{m_{i}^{2}+p_{i}^{2}}$ populates the energy states according to Fermi-Dirac or
Bose-Einstein distribution function:

$$
n_{i} \equiv n_{i}\left(E_{i}\right)=\frac{1}{\Upsilon_{i}^{-1} \exp \left(E_{i} / T\right) \pm 1},
$$

where the upper sign corresponds to Fermions and the lower one to Bosons. The fugacity $\Upsilon_{i}$ of the $i$-th hadron species is described and reduced to the valence quark properties in subsect. 9.5 below. Then the hadron species $i$ yield will correspond to the integral of the distribution function (eq. (39)) over the phase space multiplied by the hadron spin degeneracy $g_{i}=\left(2 J_{i}+1\right)$ and volume $V$

$$
\left\langle N_{i}\right\rangle \equiv\left\langle N_{i}\left(m_{i}, g_{i}, V, T, \Upsilon_{i}\right)\right\rangle=g_{i} V \int \frac{\mathrm{d}^{3} p}{(2 \pi)^{3}} n_{i}
$$

The fluctuation of the yield eq. (40) is:

$$
\left\langle\left(\Delta N_{i}\right)^{2}\right\rangle=\left.\Upsilon_{i} \frac{\partial\left\langle N_{i}\right\rangle}{\partial \Upsilon_{i}}\right|_{T, V}=g_{i} V \int \frac{\mathrm{d}^{3} p}{(2 \pi)^{3}} n_{i}\left(1 \mp n_{i}\right)
$$

It is more practical for numerical computation to express the above yields and fluctuations as an expansion in modified Bessel functions

$$
\begin{aligned}
\left\langle N_{i}\right\rangle= & \frac{g_{i} V T^{3}}{2 \pi^{2}} \sum_{n=1}^{\infty} \frac{( \pm 1)^{n-1} \Upsilon_{i}^{n}}{n^{3}} W\left(\frac{n m_{i}}{T}\right), \\
\left\langle\left(\Delta N_{i}\right)^{2}\right\rangle= & \frac{g_{i} V T^{3}}{2 \pi^{2}} \sum_{n=1}^{\infty} \frac{( \pm 1)^{n-1} \Upsilon_{i}^{n}}{n^{3}} \\
& \times\left(\begin{array}{c}
2+n-1 \\
n
\end{array}\right) W\left(\frac{n m_{i}}{T}\right) \\
W(x) \equiv & x^{2} K_{2}(x) .
\end{aligned}
$$

These expansions can be calculated to any desired accuracy; for Bosons convergence requires $\Upsilon_{i} \exp \left(-m_{i} / T\right)<1$, otherwise the expansion makes no sense. For heavy $(m \gg$ T) particles, such as charm hadrons, the Boltzmann distribution is a good approximation, i.e., it is sufficient to evaluate the first term of the expansion in eq. (42), which 
is indeed implemented in the CHARM module of SHARE to reduce computation time at no observable loss of precision.

To evaluate the yield of hadron resonance with finite width $\Gamma_{i}$, one has to weigh the yield (eq. (40)) by the resonance mass using the Breit-Wigner distribution:

$$
\begin{aligned}
\left\langle\tilde{N}_{i}^{\Gamma}\right\rangle & =\int \mathrm{d} M \frac{\Gamma_{i}}{2 \pi} \frac{\left\langle N_{i}\left(M, g_{i}, T, V, \Upsilon_{i}\right)\right\rangle}{\left(M-m_{i}\right)^{2}+\Gamma_{i}^{2} / 4} \\
& \longrightarrow\left\langle N_{i}\right\rangle \quad \text { for } \Gamma_{i} \rightarrow 0
\end{aligned}
$$

For low energy states with a large width one has to use the energy dependent resonance width, since an energy independent width implies a way too large probability of the resonance being formed with unrealistically small mass. The partial width of a decay channel $i \rightarrow j$ can be well approximated by

$$
\Gamma_{i \rightarrow j}(M)=b_{i \rightarrow j} \Gamma_{i}\left[1-\left(\frac{m_{i j}}{M}\right)^{2}\right]^{l_{i j}+1 / 2}, \quad \text { for } M>m_{i j},
$$

where $b_{i \rightarrow j}$ is the decay channel branching ratio, $m_{i j}$ is the decay threshold (i.e., sum of the decay product masses) and $l_{i j}$ is the angular momentum released in the decay. The total energy dependent width $\Gamma_{i}(M)$ is obtained using the partial widths eq. (46) for all decay channels of the resonance in question as

$$
\Gamma_{i}(M)=\sum_{j} \Gamma_{i \rightarrow j}(M)
$$

For a resonance with a finite width, we can then replace eq. (45) by

$$
\left\langle N_{i}^{\Gamma}\right\rangle=\sum_{j} \int_{m_{i j}}^{\infty} \mathrm{d} M \frac{\Gamma_{i \rightarrow j}(M)}{A_{i}} \frac{\left\langle N_{i}\left(M, g_{i}, T, V, \Upsilon_{i}\right)\right\rangle}{\left(M-m_{i}\right)^{2}+\Gamma_{i}(M)^{2} / 4},
$$

where $A_{i}$ is a normalization constant

$$
A_{i}=\sum_{j} \int_{m_{i j}}^{\infty} \mathrm{d} M \frac{\Gamma_{i \rightarrow j}(M)}{\left(M-m_{i}\right)^{2}+\Gamma_{i}(M)^{2} / 4} .
$$

Equation (48) is the form used in the program to evaluate hadron resonance yield, whenever calculation with finite width is required. Note that yield evaluation with finite width is implemented only for hadrons with no charm constituent quark; zero width $\left(\Gamma_{c_{i}}=0\right)$ is used for all charm hadrons.

\subsection{Hadron fugacity $\Upsilon_{\mathrm{i}}$ and quark chemistry}

The fugacity of hadron states defines the yields of different hadrons based on their quark content. It can be calculated from the individual constituent quark fugacities. In the most general case, for a hadron consisting of $N_{u}^{i}, N_{d}^{i}, N_{s}^{i}$ and $N_{c}^{i}$ up, down, strange and charm quarks respectively and $N_{\bar{u}}^{i}, N_{\bar{d}}^{i}, N_{\bar{s}}^{i}$ and $N_{\bar{c}}^{i}$ anti-quarks, the fugacity can be expressed as

$$
\begin{aligned}
\Upsilon_{i}= & \left(\lambda_{u} \gamma_{u}\right)^{N_{u}^{i}}\left(\lambda_{d} \gamma_{d}\right)^{N_{d}^{i}}\left(\lambda_{s} \gamma_{s}\right)^{N_{s}^{i}}\left(\lambda_{c} \gamma_{c}\right)^{N_{c}^{i}} \\
& \times\left(\lambda_{\bar{u}} \gamma_{\bar{u}}\right)^{N_{\bar{u}}^{i}}\left(\lambda_{\bar{d}} \gamma_{\bar{d}}\right)^{N_{\bar{d}}^{i}}\left(\lambda_{\bar{s}} \gamma_{\bar{s}}\right)^{N_{\bar{s}}^{i}}\left(\lambda_{\bar{c}} \gamma_{\bar{c}}\right)^{N_{\bar{c}}^{i}},
\end{aligned}
$$

where $\gamma_{f}$ is the phase space occupancy of flavor $f$ and $\lambda_{f}$ is the fugacity factor of flavor $f$. Note that we allow for non-integer quark content to account for states like $\eta$ meson, which is implemented as $\eta=0.55(u \bar{u}+d \bar{d})+0.45 s \bar{s}$ in agreement with [212]. It can be shown that for quarks and anti-quarks of the same flavor

$$
\gamma_{f}=\gamma_{\bar{f}} \quad \text { and } \quad \lambda_{f}=\lambda_{\bar{f}}^{-1}
$$

which reduces the number of variables necessary to evaluate the fugacity by half.

It is a common practice to take advantage of the isospin symmetry and to treat the two lightest quarks $(q=u, d)$ using light quark and isospin phase space occupancy and fugacity factors which are obtained via a transformation of parameters:

$$
\gamma_{q}=\sqrt{\gamma_{u} \gamma_{d}}, \quad \gamma_{3}=\sqrt{\frac{\gamma_{u}}{\gamma_{d}}}
$$

with straightforward backwards transformation

$$
\gamma_{u}=\gamma_{q} \gamma_{3}, \quad \gamma_{d}=\gamma_{q} / \gamma_{3},
$$

and similarly for the fugacity factors

$$
\begin{aligned}
& \lambda_{q}=\sqrt{\lambda_{u} \lambda_{d}}, \quad \lambda_{3}=\sqrt{\frac{\lambda_{u}}{\lambda_{d}}}, \\
& \lambda_{u}=\lambda_{q} \lambda_{3}, \quad \lambda_{d}=\lambda_{q} / \lambda_{3} .
\end{aligned}
$$

Chemical potentials are closely related to fugacity; one can express an associated chemical potential $\mu_{i}$ for each hadron species $i$ via

$$
\Upsilon_{i}=e^{\mu_{i} / T}
$$

It is more common to express chemical potentials related to conserved quantum numbers of the system, such as baryon number $B$, strangeness $s$, third component of isospin $I_{3}$ and charm $c$ :

$$
\begin{aligned}
\mu_{B} & =3 T \log \lambda_{q}, \\
\mu_{S} & =T \log \lambda_{q} / \lambda_{s}, \\
\mu_{I_{3}} & =T \log \lambda_{3}, \\
\mu_{C} & =T \log \lambda_{c} \lambda_{q} .
\end{aligned}
$$

Notice the inverse, compared to intuitive definition of $\mu_{S}$, which has a historical origin and is a source of frequent mistakes.

\subsection{Resonance decays}

The hadron yields observed include the post-hadronization decays of in general free streaming hadron states only a few are stable enough to reach detectors. In fact 
heavier resonances decay rapidly after the freeze-out and feed lighter resonances and "stable" particle yields. The final stable particle yields are obtained by allowing all resonances to decay sequentially from the heaviest to the lightest and thus correctly accounting for resonance cascades.

The observable yield of each hadron $i$ including into the study the resonances populated by more massive resonances, is then a combination of primary production and feed from resonance decays

$$
\left\langle N_{i}\right\rangle=\left\langle N_{i}\right\rangle_{\text {primary }}+\sum_{j \neq i} B_{j \rightarrow i}\left\langle N_{j}\right\rangle,
$$

where $B_{j \rightarrow i}$ is the probability (branching ratio) that particle $j$ will decay into particle $i$. Applied recursively, eq. (61) generated the model result that corresponds to the experimentally observable yields of all hadrons, "stable" and unstable resonances, which are often of interest.

The SHARE program includes for non-charm hadrons all decay channels with branching ratio $\geq 10^{-2}$ in data tables. To attain the parallel level of precision for the higher number of charm hadron decays (a few hundred (!) in some cases) with small branching ratios required to set the acceptance for decay channels at a branching ratio $\geq 10^{-4}$. Since charm hadrons in many cases decay into more than three particles, a more complex approach in implementing them had to be used [209].

There is still a lot of uncertainty regarding charm decay channels. Some of them are experimentally difficult to confirm, but required and had to be estimated based on symmetries. For example, a measured $\Lambda_{c}^{+}$decay channel

$$
\Lambda_{c}^{+} \rightarrow p \bar{K}^{0} \pi^{0} \quad(3.3 \pm 1.0) \%
$$

is complemented by the unobserved isospin symmetric channel

$$
\Lambda_{c}^{+} \rightarrow n \bar{K}^{0} \pi^{+} \quad(3.3 \pm 1.0) \%,
$$

with the same branching ratio.

The influence of resonance feed-down on fluctuations is the following:

$$
\left\langle\left(\Delta N_{j \rightarrow i}\right)^{2}\right\rangle=B_{j \rightarrow i}\left(\mathcal{N}_{j \rightarrow i}-B_{j \rightarrow i}\right)\left\langle N_{j}\right\rangle+B_{j \rightarrow i}^{2}\left\langle\left(\Delta N_{j}\right)^{2}\right\rangle .
$$

The first term corresponds to the fluctuations of the mother particle $j$, which decays into particle $i$ with branching ratio $B_{j \rightarrow i} . \mathcal{N}_{j \rightarrow i}$ is the number of particles $i$ produced in the decay of $i$ (inclusive production) so that $\sum_{i} B_{j \rightarrow i}=\mathcal{N}_{j \rightarrow i}$. For nearly all decays of almost all resonances $\mathcal{N}_{j \rightarrow i}=1$, however, there are significant exceptions to this, including the production of multiple $\pi^{0}$, such as $\eta \rightarrow 3 \pi^{0}$. The second term in eq. (64) corresponds to the fluctuation in the yield of the mother particle (resonance).

\section{Hadrons from QGP: What do we learn?}

A comparison of lattice results with freeze-out conditions were shown in fig. 9. The band near to the temperature axis displays the lattice estimate for $T_{\mathrm{H}}$ presented in ref. [71], $T_{\mathrm{H}}=147 \pm 5 \mathrm{MeV}$. As fig. 9 demonstrates, many SHM are in more or less severe conflict with this value of $T_{\mathrm{H}}$. The model SHARE we detailed in previous sect. 9 is, however, in excellent agreement. One of the reasons to write this review is to highlight how the change in understanding of $T_{\mathrm{H}}$ impacts the resultant choice that emerges in terms of SHM applicability.

The SHARE toolbox permits a complete analysis of any sufficiently large family set of particle yields that is consistently presented in terms of a given reaction energy and participant number class $N_{\text {part }}$. Especially as a function of $N_{\text {part }}$ this is not always the case, whence some interpolation of data is a part of the analysis. We do not discuss this practical issue further here. The material selected for presentation is not comprehensive and it is only representative of the work manifestly consistent with fig. 9 .

Another criterion that we use is to focus on particle yields only. Doing this, we need to mention upfront the work of Begun, Florkowski and Rybczynski [213,214] which applies the same non-equilibrium methods in an ambitious effort to describe all LHC particle spectra and does this with good success. These results are directly relevant to our study of LHC data presenting complementing information that confirms our statistical parameter determination.

We will also show, by an example, some of the issues that have affected the SHM analysis carried out by another group.

\subsection{Hadron source bulk properties before LHC}

Among the important features built into the SHARE program is the capability to fully describe the properties of the fireball that produces the particles analyzed. This is not done in terms of produced particles: each carries away "content", such as the energy of the fireball. We evaluate and sum all fractional contributions to the fireball bulk properties from the observed and, importantly, unobserved particles, predicted by the fit in their abundance. The energy content is only thermal, as we eliminate using yields the effect of expansion flow on the spectra, i.e. the dynamical collective flow energy of matter. Thus the energy content we compute is the "comoving" total thermal energy.

Given the large set of parameters that SHARE makes available we fit all particles well and thus the physical properties that we report are rather precise images of the observed particle yields. The question what the SHM parameters mean does not enter the discussion at all. If a measurement error has crept in then our results would look anomalous when inspected as a function of collision energy or collision centrality.

The fit of SHM parameters then provides an extrapolation from the measured particle abundances to unmeasured yields of all particles known and listed in SHARE tables. Most of these are of no great individual relevance, being too massive. The bulk properties we report here are, for the most part, defined by particles directly observed. We expect smooth lines describing the fireball properties as a function of $\sqrt{s_{\mathrm{NN}}}$, the CM energy 


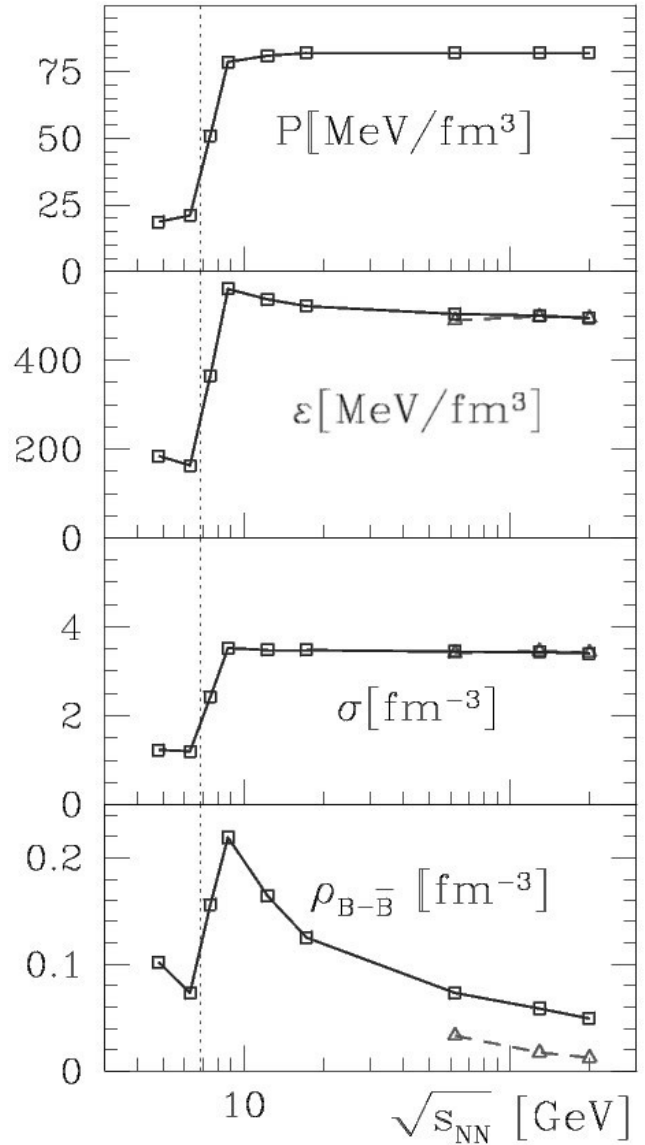

Fig. 28. Fireball bulk properties in the SPS and RHIC energy domain, see text. Update of results published in ref. [7].

per pair of nucleons and/or as function of collision centrality class $N_{\text {npart }}$. Appearance of discontinuous behavior as a function of $\sqrt{s_{\mathrm{NN}}}$ can indicate a change related to QGP formation

In fig. 28 we see in the SPS and RHIC energy domain for most head-on collisions, from top to bottom, the pressure $P$, energy density $\varepsilon$, the entropy density $\sigma$, and the net baryon density $\rho_{\mathrm{B}-\overline{\mathrm{B}}}$. SPS and RHIC $4 \pi$ data were used, for RHIC range also results obtained fitting $\mathrm{d} N / \mathrm{d} y$ are shown by the dashed line, particles originating in a volume $\mathrm{d} V / \mathrm{d} y, y \in\{-0.5,0.5\}$. Only for the baryon density can we recognize a serious difference; the baryon density in the central rapidity region seems to be a factor 5 below average baryon density. Not shown is the change in the fitted volume, which is the one changing quantity (aside of $\left.\rho_{\mathrm{B}-\overline{\mathrm{B}}}\right)$. Volume grows to accommodate the rapid rise in particle multiplicity with the available energy.

Figure 28 shows exciting features worth further discussion. There can be no doubt that over a relatively small domain of collision energy - in laboratory frame, between 20 and $40 A \mathrm{GeV}$ (SPS projectile per nucleon energies) and in $\mathrm{CM}$ frame $\sqrt{s_{\mathrm{NN}}} \in\{6.5,7.5\} \mathrm{GeV}$ per nucleon pairthe properties of the fireball change entirely. Is this a signal of the onset of new physics? And if so why, is this happening at this energy? Though this experimental result has been recognized for nearly 10 years now, ref. [7] and private communication by M. Gaździcki, I have no clear answer to offer to these simple questions.

We find a peak in the net baryon density, bottom frame of the fig. 28. The $K^{+} / \pi^{+}$peaking, fig. 23, discussed in subsect. 6.3 seems to be related to the effect of baryon stopping, perhaps a rise as function of $\sqrt{s_{\mathrm{NN}}}$ in stopping power at first, when color bonds are broken, and a more gradual decline with increasing energy. But what makes quarks stop just then? And why do they decide to stop less at higher energy, instead "shooting through"? Note that a possible argument that a decrease in baryon density is due to volume growth is not right considering that the thermal energy density $\epsilon$, and the entropy density $\sigma$ remain constant above the threshold in collision energy.

I would argue that when first color bonds are melted, gluons are stopped while quarks are more likely to run out. That would agree with our finding in context of strangeness production, see ref. [16], that despite similar looking matrix elements in perturbative QCD, gluons are much more effective in making things happen due to their "high" adjoint representation color charge; the best analogy would be to say that gluons have double-color charge. The high gluon density at first manages to stop some quarks but the probability decreases with increasing energy. It is remarkable how fast the dimensionless $\rho_{\mathrm{B}-\overline{\mathrm{B}}} / \sigma \equiv b / S$ drops. This expresses the ability to stop quarks normalized to the ability to produce entropy.

Seeing all these results, one cannot but ask what the total abundance of strange quark pairs will do. Before the discussion of results seen in fig. 29 it is wise to read the conclusions in ref. [16] where in 1983 the overall strangeness yield enhancement alone was not predicted to be a striking signature. In fig. 29 ratios are shown, in the top frame: the pair strangeness abundance $s$ per net baryon abundance $b$; per entropy in the middle frame; and in the bottom frame we see the energy cost in $\mathrm{GeV}$ to make a strange quark pair, $E / s$; mind you that this energy is the final state thermal fireball energy.

We see in fig. 29 that the $s / b$ ratio is smooth. This means that strangeness production takes off where baryon stopping takes off, being in the QGP attributed range of $\sqrt{s_{N N}}$ faster than rise in entropy production. And, the energy cost of a pair seems to be very low at high energy: only $5-6$ times the energy that the pair actually carries by itself, and this factor reflects accurately on how abundant strangeness is in comparison to all the other constituents of the QGP fireball. This by itself clearly indicates that the yield converges to chemical QGP equilibrium. The clear break in the cost of making a strange quark pair near $30 \mathrm{GeV}$ energy shows the threshold above which strangeness, as compared to other components, becomes an equal fireball partner.

Our analysis thus shows: a) There is an onset of baryon transparency and entropy production at a very narrowly defined collision energy range. b) Beyond this threshold in collision energy the hadronization proceeds more effectively into strange antibaryons. c) The universality of hadronization source properties, such as energy density, or entropy density above the same energy threshold, suggest as explanation that a new phase of matter hadronizes. 


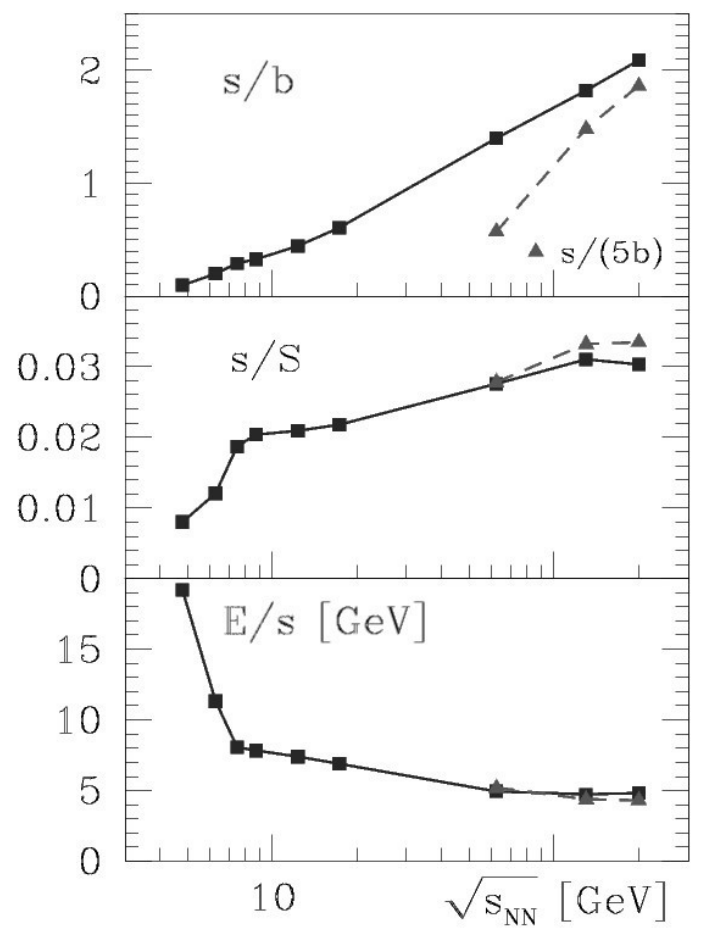

Fig. 29. Strangeness pair production $s$ yield from SPS and RHIC as a function $\sqrt{s_{N N}}$ : yield normalized by net baryon abundance $b$ in top frame, entropy $S$ in middle frame. At bottom the energy cost to produce strangeness. Total particle yields, except for $\mathrm{d} N / \mathrm{d} y$ results shown as dashed lines in the RHIC energy range. Update of results published in ref. [7].

There is little doubt considering these cornerstone analysis results that at SPS at and above the projectile energy of $30 \mathrm{AGeV}$ we produced a rapidly evaporating (hadronizing) drop of QGP. The analysis results we presented for the properties of the fireball leave very little space for other interpretation. The properties of the QGP fireball created in the energy range of $30-156 A \mathrm{GeV} \mathrm{Pb}$ $\mathrm{Pb}$ collisions at CERN are just the same as those obtained for RHIC beam energy scan, see end of subsect. 6.3.

\subsection{LHC SHM analysis}

We consider now LHC results obtained at $\sqrt{s_{N N}}=$ $2760 \mathrm{GeV}$ as a function of participant number $N_{\text {part }}$, sect. 9.3 and compare with an earlier similar analysis of STAR results available at $\sqrt{s_{\mathrm{NN}}}=62 \mathrm{GeV}[76]$. In comparison, there is a nearly a factor 50 difference in collision energy. The results presented here for LHC are from the ALICE experiment as analyzed in refs. [73-75]. The experimental data inputs were discussed extensively in these references, the data source includes refs. [87,153,215,216]. The analysis of hadron production as a function of participant number $N_{\text {part }}$ at RHIC and LHC proceeds in essentially the same way as already described. The results here presented were obtained without the contribution of charmed hadrons.

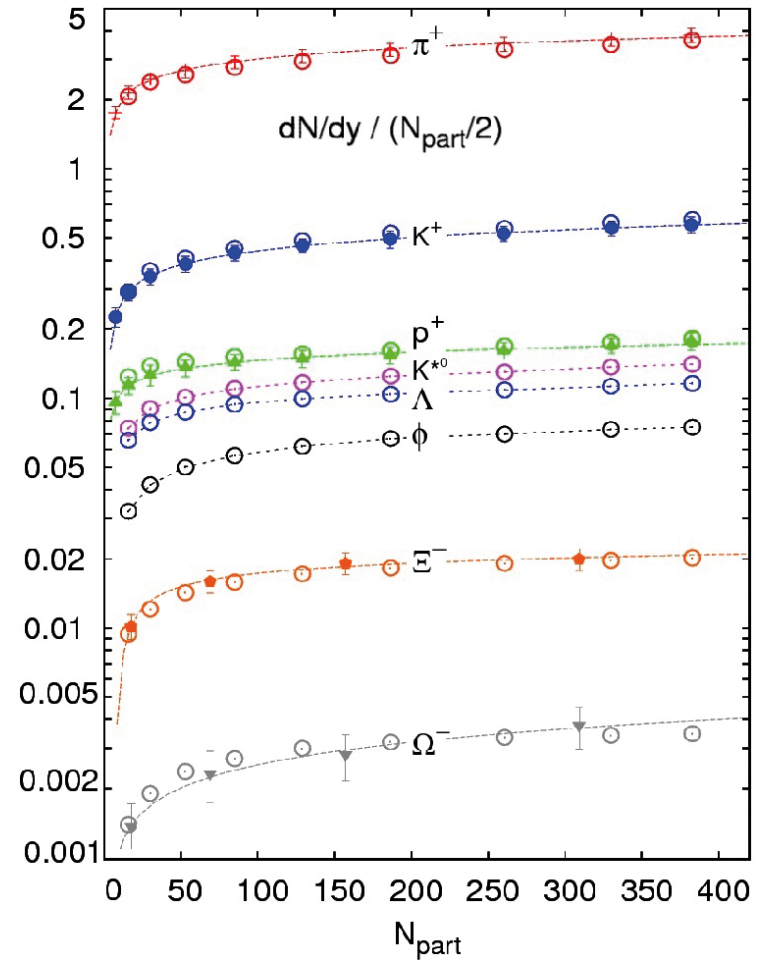

Fig. 30. LHC experimental data measured by the ALICE experiment in $\mathrm{Pb}-\mathrm{Pb}$ collisions at $\sqrt{s_{N N}}=2.76 \mathrm{TeV}$ as function of centrality described by $N_{\text {part }}$, normalized by $N_{\text {part }} / 2$. Results adapted from refs. [73-75].

Given the large set of available SHARE parameters all particles are described very well, a non-complete example of the data included is seen in fig. 30 . Note that the central rapidity yields are divided by $N_{\text {part }} / 2$; that is they are per nucleon pair as in $p p$ collisions. This also means that our fit spans a range of a yield of $\mathrm{d} N_{\Omega} \simeq 10^{-4}$ for the most peripheral collisions to $\mathrm{d} N_{\pi} \simeq 2000$ for the most central collisions, thus more than 7 orders of magnitude alone of particles shown in fig. 30 .

About three orders of magnitude of the large range of yields $\mathrm{d} N / \mathrm{d} y$ that are fitted are absorbed into the rapidly changing volume $\mathrm{d} V / \mathrm{d} y$ from which these particles emerge, see fig. 31. Note that this result is already reduced by the factor $N_{\text {part }} / 2$; thus this is volume per colliding nucleon pair. For RHIC we see that this is a rather constant value to which the LHC results seem to converge for small value of $N_{\text {part }}$. However for large $N_{\text {part }}$ at LHC the specific volume keeps growing. Keep in mind that the interpretation of $\mathrm{d} V / \mathrm{d} y$ is difficult and a priori is not geometric, see subsect. 9.2.

The corresponding LHC and RHIC chemical freeze-out temperature $T$, fig. 32, varies both at RHIC and LHC in the same fashion with larger values found for smaller hadronization volumes. This is natural, as scattering length for decoupling must be larger than the size of the system and thus the more dense hotter condition is possible for the smaller fireball. One can also argue with the same outcome that the rapid expansion of the larger fireball can lead to stronger supercooling of QGP which directly trans- 


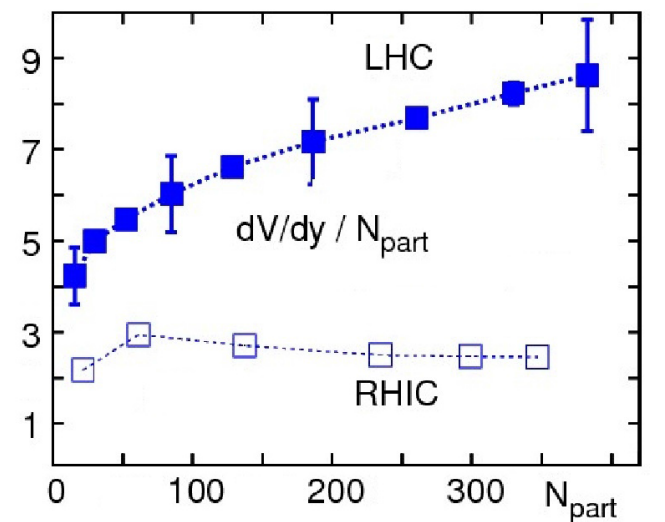

Fig. 31. The source volume $\mathrm{d} V / \mathrm{d} y$ at $\sqrt{s_{N N}}=2.76 \mathrm{TeV}$, normalized by number of nucleon pairs $N_{\text {part }} / 2$, as a function of the number of participants $N_{\text {part }}$. For comparison, a similar STAR $\sqrt{s_{\mathrm{NN}}}=62 \mathrm{GeV}$ data analysis is shown. Results adapted from refs. [73-75].

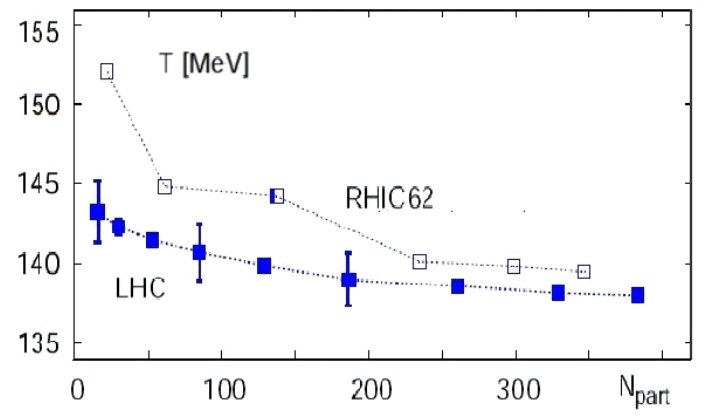

Fig. 32. The chemical freeze-out temperature $T$ at $\sqrt{s_{N N}}=$ $2.76 \mathrm{TeV}$, as a function of the number of participants $N_{\text {part }}$, lines guide the eye. Results adapted from refs. [73-75].

forms into free-streaming hadrons. The possibility of direct QGP hadronization is supported by the strong chemical non-equilibrium with $\gamma_{q}>1, \gamma_{s}>1$ for all collision centralities. These results are seen in fig. 33 .

In fig. 34 we see the physical properties of the fireball as obtained by the same procedure as discussed in subsect. 10.1. With increasing participant number all these bulk properties decrease steadily. This is the most marked difference to the RHIC results. We should here remember that the hadronization volume at LHC given the greater total energy content of the fireball is much greater and thus the dynamics of fireball expansion should be different.

Results seen in fig. 34 show a remarkable universality, both when LHC is compared to RHIC, and as a function of centrality; variation as a function of $N_{\text {part }}$ is much smaller than that seen in particle yields in fig. 30 (keep in mind that these results are divided by $\left.N_{\text {part }} / 2\right)$. The universality of the hadronization condition is even more pronounced when we study, see fig. $35,(\varepsilon-3 P) / T^{4}$, the interaction measure $I_{\mathrm{m}}$ eq. (11) (compare subsect. 2.5, fig. 7).

We observe that the lattice-QCD maximum from fig. 7 $(\varepsilon-3 P) / T^{4}$ falls right into the uncertainty band of this result. Only for $\gamma_{q} \simeq 1.6$ and $\gamma_{s} \simeq 2$ a high value for $I_{\mathrm{m}}$ shown in fig. 35 can be obtained. The equilibrium hadron

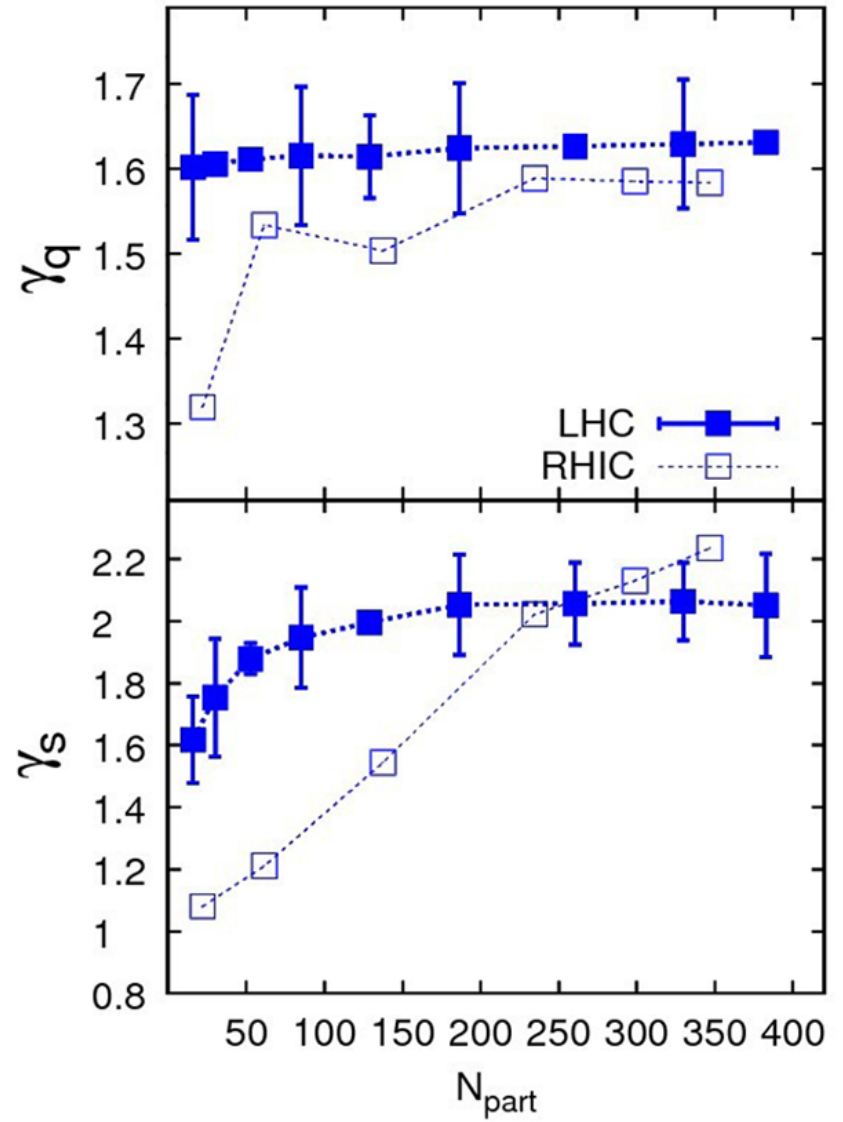

Fig. 33. Light quark $\gamma_{q}$ and strange quark $\gamma_{s}$ fugacities at $\sqrt{s_{N N}}=2.76 \mathrm{TeV}$ as a function of the number of participants $N_{\text {part }}$, lines guide the eye. Results adapted from refs. [73-75].

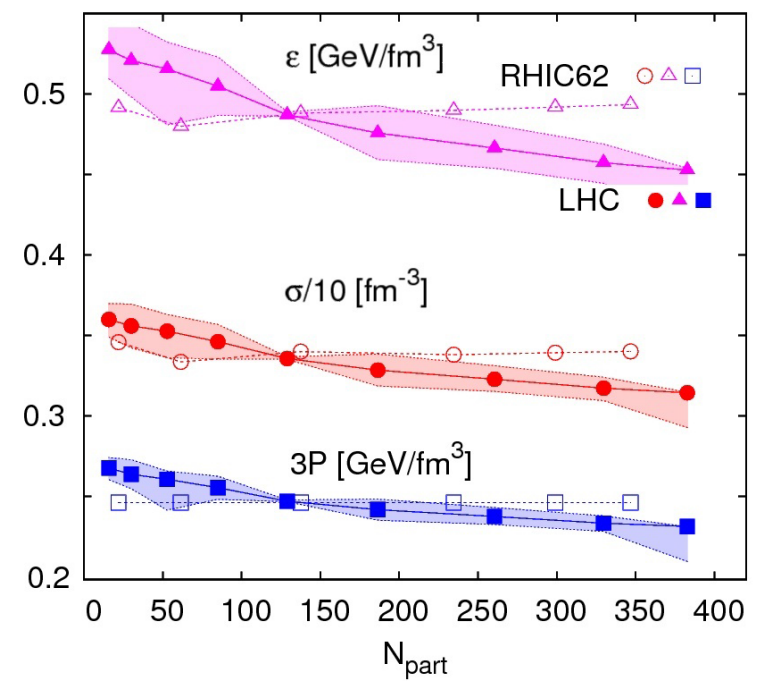

Fig. 34. From top to bottom as function of centrality described by $N_{\text {part }}$ : energy density $\varepsilon$, entropy density $\sigma$ reduced by a factor 10 to fit in figure, and $3 P$ at $\sqrt{s_{N N}}=2.76 \mathrm{TeV}$. The dotted line are RHIC $\sqrt{s_{\mathrm{NN}}}=62 \mathrm{GeV}$ analysis results not showing the (larger) error band. Results adapted from refs. [73$75]$. 


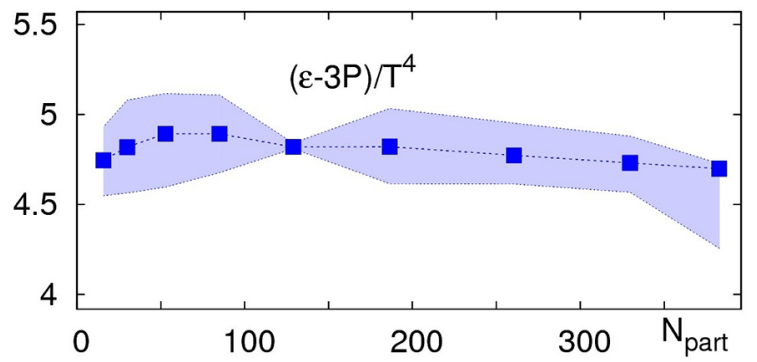

Fig. 35. Hadronization universality: the interaction measure $(\varepsilon-3 P) / T^{4}$ evaluated at hadronization condition of the hadron fireball created in $\sqrt{s_{N N}}=2.76 \mathrm{TeV} \mathrm{Pb}-\mathrm{Pb}$ collisions as a function of centrality described by $N_{\text {part }}$. Results adapted from refs. [73-75].

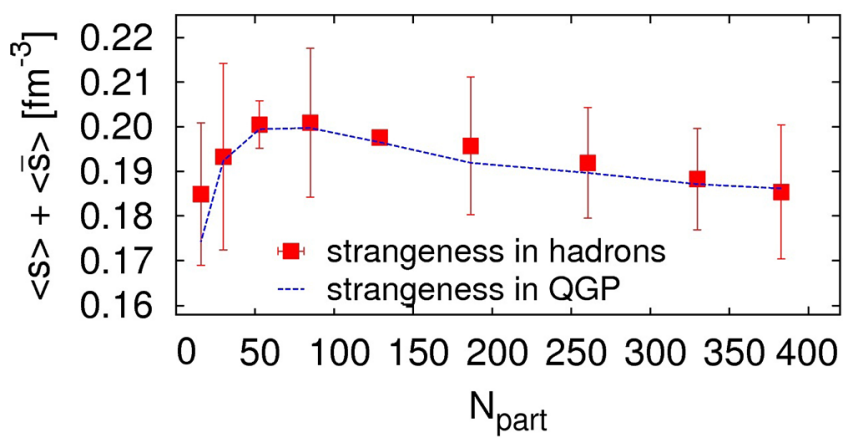

Fig. 36. $\langle s\rangle+\langle\bar{s}\rangle$ strangeness density measured in the hadron phase (red squares) as a function of centrality described by $N_{\text {part }}$. The dashed (blue) line is a fit with strangeness in the QGP phase, see text. Results adapted from refs. [73-75].

gas results are about a factor 3 smaller in the relevant domain of temperature.

Turning our attention now to strangeness: In the most central $5 \% \mathrm{~Pb}-\mathrm{Pb}$ collisions at the LHC2760, a total of $\mathrm{d} N_{s \bar{s}} / \mathrm{d} y \simeq 600$ strange and anti-strange quarks per unit of rapidity is produced. For the more peripheral collisions the rise of the total strangeness yield is very rapid, as both the size of the reaction volume and within the small fireball the approach to saturation of strangeness production in the larger QGP fireball combine.

It is of considerable interest to understand the magnitude of strangeness QGP density at hadronization. We form a sum of all (strange) hadron multiplicities $\mathrm{d} N_{h} / \mathrm{d} y$ weighting the sum with the strange content $-3 \leq n_{s}^{h} \geq 3$ of any hadron $h$ and include hidden strangeness, to obtain the result shown in fig. 36 . Within the error bar the result is a constant; strange quarks and antiquarks in the fireball are $20 \%$ more dense than are nucleons bound in nuclei.

However, is this $\langle s\rangle+\langle\bar{s}\rangle$ strangeness density shown by error bars in fig. 36 a density related to QGP? To give this result a quantitative QGP meaning we evaluate QGP phase strangeness density at a given $T$, see eq. (42)

$$
\begin{aligned}
s\left(m_{s}, T ; \gamma_{s}^{\mathrm{QGP}}\right)= & -\frac{g T^{3}}{2 \pi^{2}} \sum_{n=1}^{\infty}\left(-\gamma_{s}^{\mathrm{QGP}}\right)^{n} \\
& \times \frac{1}{n^{3}}\left(\frac{n m_{s}}{T}\right)^{2} K_{2}\left(\frac{n m_{s}}{T}\right),
\end{aligned}
$$

where $m_{s}$ is the (thermal) strange quark mass, $\gamma_{s}^{\mathrm{QGP}}$ is the phase space occupancy: here the superscript QGP helps to distinguish from that measured in the hadronization analysis as $\gamma_{s}$, used without a superscript. The degeneracy is $g=12=2_{\text {spin }} 3_{\text {color }} 2_{\mathrm{p}}$ where the last factor accounts for the presence of both quarks and antiquarks.

In central LHC collisions, the large volume (longer lifespan) also means that strangeness approaches saturated yield in the QGP. In peripheral collisions, the short lifespan of the fireball may not be sufficient to reach chemical equilibrium. Therefore we introduce a centrality dependent strangeness phase space occupancy $\gamma_{s}^{\mathrm{QGP}}\left(N_{\text {part }}\right)$ which is to be used in eq. (65).

A model of the centrality dependence of $\gamma_{s}^{\mathrm{QGP}}\left(N_{\text {part }}\right)$ is not an important consideration, as the yield for $N_{\text {part }}>30$ is nearly constant. The value of strangeness density requires $m_{s}=299 \mathrm{MeV}$ in a QGP fireball at hadronization. For $m_{s} \simeq 140 \mathrm{MeV}$ (mass at a scale of $\mu \simeq$ $2 \pi T \simeq 0.9 \mathrm{GeV}) . \gamma_{s \text { final }}^{\mathrm{QGP}} \simeq 0.77$ is found. The higher value of $m_{s}$ makes more sense in view of the need to account for the thermal effects. Thus we conclude that for $N_{\text {part }}>30$ the fireball contains QGP chemical equilibrium strangeness abundance, with strangeness thermal mass $m_{s}=299 \mathrm{MeV}$ [75].

The ratio of strangeness to entropy is easily recognized to be, for QGP, a measure of the relative number of strange to all particles — adding a factor $\simeq 4$ describing the amount of entropy that each particle carries. Thus a QGP source will weigh in with ratio $s / S \simeq 0.03$ [217]. This is about factor 1.4 larger than one computes for hadron phase at the same $T$, and this factor describes the strangeness enhancement effect in abundance which was predicted to be that small, see ref. [16]. However, if a QGP fireball was formed we do expect a rather constant $s / S$ as a function of $N_{\text {part }}$.

\subsection{Earlier work}

Results of SHM that provide freeze-out $T$ well above $T_{\mathrm{H}}$ seen in fig. 9 should today be considered obsolete. As an example let us enlarge here on the results of ref. [218] which would be marked in fig. 9 GSI-RHIC at $T \simeq 174 \pm 7$, $\mu_{\mathrm{B}} \simeq 46 \pm 5 \mathrm{MeV}$ corresponding to a fit of $\sqrt{s_{\mathrm{NN}}}=$ $130 \mathrm{GeV}$ RHIC results (but the point is not shown above the upper $T$ margin). This reference assumes full chemical equilibrium. They draw attention to agreements with other results and expectations, both in their conclusions, as well as in the body of their text, verbatim:

"The chemical freeze-out temperature $T_{f} \simeq 168 \pm$ $2.4 \mathrm{MeV}$ found from a thermal analysis of experimental data in $\mathrm{Pb}-\mathrm{Pb}$ collisions at SPS is remarkably consistent within error with the critical temperature $T_{c} \simeq 170 \pm 8 \mathrm{MeV}$ obtained from lattice Monte Carlo simulations of QCD at vanishing baryon density [15] and [16]"

Their lattice references are [15, from the year 2001] [219] and [16, from the year 1999] [220]. The two references disagree in regard to value of $T_{\mathrm{H}}$, verbatim: 
(1999) "If the quark mass dependence does not change drastically closer to the chiral limit the current data suggest $T_{c} \simeq(170-190) \mathrm{MeV}$ for 2 -flavor QCD in the chiral limit. In fact, this estimate also holds for 3-flavor QCD.".

(2001) "The 3-flavor theory, on the other hand, leads to consistently smaller values of the critical temperature,... 3 flavor QCD: $T_{c}=(154 \pm$ 8) MeV."

While the authors of ref. [218] were clearly encouraged by the 1999 side remark in ref. [220] about 3 flavors, they also cite in the same breath the correction [219] which renders their RHIC SHM fit invalid: for a lattice result $T_{\mathrm{H}}=154 \pm 8 \mathrm{MeV}$ chemical freeze-out at $T \simeq 174 \pm 7 \mathrm{MeV}$ seems inconsistent since $T<T_{\mathrm{H}}$ strictly.

When reading ref. [218] in Spring 2002 I further spotted that it is technically wanting. Namely, the experimental $\bar{\Xi} / \Xi$ ratio used in the paper predicts a value $\mu_{\Xi}=\mu_{\mathrm{B}}-2 \mu_{\mathrm{S}}=18.8 \mathrm{MeV}$ while the paper determines from this ratio a value $\mu_{\Xi}=\mu_{\mathrm{B}}-2 \mu_{\mathrm{S}}=9.75 \mathrm{MeV}$. In conclusion: the cornerstone manuscript of the GSI group is at the time of publication inconsistent with the lattice used as justification showing chemical freeze-out $T>T_{\mathrm{H}}$ by $20 \mathrm{MeV}$, and its computational part contains a technical mistake. But, this paper had a "good" confidence level.

The key argument of the paper is that $\chi^{2} /$ dof $\simeq 1$. However, $\chi^{2}$ depends in that case on large error bars in the initial $130 \mathrm{GeV}$ RHIC results. Trusting $\chi^{2}$ alone is not appropriate to judge a fit result ${ }^{12}$. A way to say this is to argue that a fit must be "confirmed" by theory, and indeed that is what ref. [218] claimed, citing ref. [219] which however, provided a result in direct disagreement.

Thus we can conclude that ref. [218] at time of publication had already proved itself wrong. And while humanum errare est, students lack the experience to capture theirs effectively. Today this work is cited more than 500 times - meaning that despite the obvious errors and omissions it has entered into the contemporary knowledge base. Its results confuse the uninitiated deeply. These results could only be erased by a direct withdrawal note by the authors.

\subsection{Evaluation of LHC SHM fit results}

The chemical non-equilibrium SHM describes very well all available LHC-2760 hadron production data obtained in a wide range of centralities $N_{\text {part }}$, measured in the CM within the rapidity interval $-0.5 \leq 0 \leq 0.5$. A value of freeze-out temperature that is clearly below the range for $T_{\mathrm{H}}$ reported in fig. 9 for lattice-QCD arises only when accepting a full chemical non-equilibrium outcome. Chemical non-equilibrium is expected for the hadron phase space

\footnotetext{
12 Hagedorn explained the abuse of $\chi^{2}$ as follows: he carried an elephant and mouse transparency set, showing how both transparencies are fitted by a third one comprising a partial picture of something. Both mouse and elephant fitted the something very well. In order to distinguish mouse from elephant, one needs external scientific understanding; in his example, the scale, was required.
}

if QGP fireball was in chemical equilibrium. In that sense, theory supports the finding, and this result also has a very good $\chi^{2} /$ ndf $<1$ for all collision centralities.

The value of the ratio $p /\left.\pi\right|_{\text {experiment }}=0.046 \pm$ $0.003[215,87]$ is a LHC result that any model of particle production in RHI collisions must agree with. The value $p / \pi \simeq 0.05$ is a natural outcome of the chemical non-equilibrium fit with $\gamma_{q} \simeq 1$.6. This result was predicted in ref. [221]: $p /\left.\pi\right|_{\text {prediction }}=0.047 \pm 0.002$ for the hadronization pressure seen at RHIC and SPS $P=$ $82 \pm 5 \mathrm{MeV} / \mathrm{fm}^{3}$. Chemical equilibrium model predicts and fits a very much larger result. This is the so-called proton anomaly; there is no anomaly if one does not dogmatically prescribe chemical equilibrium conditions.

A recent study of the proton spectra within the freezeout model developed in Krakow confirms the chemical non-equilibrium [213]: In fig. 37 we show a comparison between a spectral fit of pions, kaons and protons within the equilibrium and non-equilibrium approaches. These results show the strong overprediction of soft protons and some overprediction of kaons that one finds in the equilibrium model. The chemical non-equilibrium model provides an excellent description of this key data.

Further evidence for the chemical non-equilibrium outcome of SHM analysis arises from the universality of hadronization at LHC, RHIC and SPS: the bulk properties of the fireball that we determine are all very similar to each other. This can be seen by comparing RHIC-SPS results presented in fig. 28 with those shown in fig. 34 for LHC-RHIC.

This universality includes the strangeness content of the fireball. The LHC particle multiplicity data has relatively small errors, allowing establishment of relatively precise results. The strong non-equilibrium result $\gamma_{s} \rightarrow 2$ seen in fig. 33 allows the description of the large abundance of multi-strange hadrons despite the relatively small value of freeze-out temperature. The value of light quark fugacity, $\gamma_{q} \rightarrow 1.6$ allows a match in the high entropy content of the QGP fireball with the $\gamma_{q}$ enhanced phase space of hadrons, especially mesons. As noted above, this effect naturally provides the correct $p / \pi$ ratio at small $T$.

There are two noticeable differences that appear in comparing RHIC62 to LHC2760 results; in fig. 31 we see as a function of centrality the specific volume parameter $(\mathrm{d} V / \mathrm{d} y) / N_{\text {part }}$. The noticeable difference is that at RHIC this value is essentially constant, while at LHC there is clearly a visible increase. One can associate this with a corresponding increase in entropy per participant, implying that a novel component in entropy production must have opened up in the LHC energy regime. This additional entropy production also explains why at LHC the maximum value of specific strangeness pair yield per entropy is smaller when compared to RHIC62 for most central collisions, see fig. 38 .

The results found in the LHC-SHM analysis characterize a fireball that has properties which can be directly compared with results of lattice-QCD, and which have not been as yet reported; thus this analysis offers a prediction which can be used to verify the consistency of SHM 


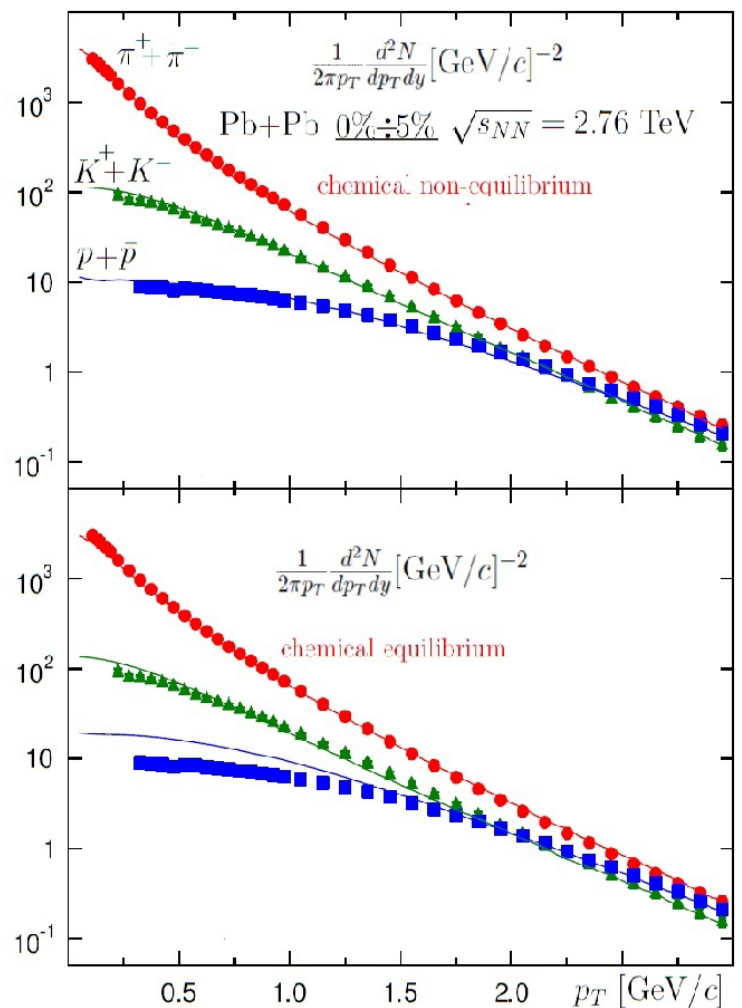

Fig. 37. Data: most central spectra of pions, kaons and protons from ALICE experiment $[87,88]$ as a function of $p_{\perp}$. Lines: (Top) the non-equilibrium model for parameters of this review; (bottom) the outcome with equilibrium constraint. Figure adapted from ref. [213].

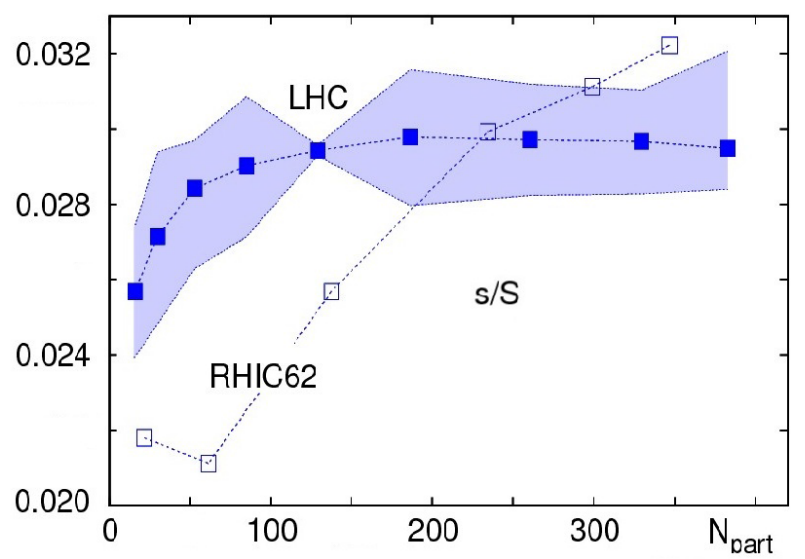

Fig. 38. Ratio of strangeness pair yield to entropy $s / S$ as function of collision centrality described by $N_{\text {part }}$. Results adapted from refs. [73-75].

results with lattice. For example, note the dimensionless ratio of the number of strange quark pairs with entropy $s / S \rightarrow 0.03$. Since strange quarks have a mass scale, this ratio can be expected to be a function of temperature in lattice-QCD evaluation. The interesting question is, at what $T$ will lattice obtain this strangeness hadronization condition $s / S=0.03$ ?

Further, there is a variation as a function of centrality seen in fig. $38 ; s / S$ decreases with decreasing $N_{\text {part }}$. Seeing that freeze-out $T$ increases, compare fig. 32 , we obtain the prediction that as freeze-out $T$ increases, $s / S$ decreases. On first sight this is counterintuitive as we would think that at higher $T$ there is more strangeness. This is an interesting behavior that may provide an opportunity to better understand the relation of the freeze-out analysis with lattice-QCD results.

\section{Comments and conclusions}

To best of my knowledge nobody has attempted a synthesis of the theory of hot hadronic matter, lattice-QCD results, and the statistical hadronization model. This is done here against the backdrop of the rich volume of soft hadron production results that have emerged in the past 20 years in the field of RHI collisions, covering the entire range of SPS, RHIC and now LHC energy, ranging a factor 1000 in $\sqrt{s_{N N}}$.

This is certainly not the ultimate word since we expect new and important experimental results in the next few years: LHC-ion operations will reach near maximum energy by the end of 2015; further decisive energy increases could take another lifespan. On the other energy range end, we are reaching out to the domain where we expect the QGP formation energy threshold, both at RHIC-BES, and at SPS-NA61. The future will show if new experimental facilities today in construction and/or advanced planning will come online within this decade and join in the study of QGP formation threshold. Such plans have been made both at GSI and at DUBNA laboratories.

In order for this report to be also a readable RHI collision introduction, I provided pages distributed across the manuscript, suitable both for students starting in the field, and readers from other areas of science who are interested in the topic. I realize that most of technical material is not accessible to these two groups, but it is better to build a bridge of understanding than to do nothing. Moreover, some historical considerations may be welcome in these circles.

I did set many of the insights into their historical context which I have witnessed personally. In my eyes understanding the history, how topics came to be looked at the way they are, helps both the present generation to learn what we know, and the future generation in resolving the misunderstandings that block progress.

For this reason I felt that many insights that I needed to develop could be presented here equally well in the format of work done many years ago. Therefore, in the jointly published refs. $[15,16]$ I present two unpublished reports from conference proceedings, long gone from library shelves, which I think provide quite appropriate background material for this report. Perhaps I should have abridged this bonus material to omit a few obsolete developments, and/or to avoid duplication across these $80+$ pages. However, any contemporary change will modify in a damaging way the historical context of these presentations.

There was a very special reason to prepare this report now. I took up this task after I finished editing a book to honor 50 years of Hagedorn remarkable achievements, 
ref. [1]. Given the historical context and the target of interest in the book being also a person, I could not inject there all the results that the reader sees in these pages. The overlap between this work and ref. [1] is small, mostly when I describe how the field developed historically before 1985.

This background material as presented here is more extensive compared to ref. [1] as I can go into the detail without concern about the contents balance of an edited book. For this reason a reader of ref. [1] should look at this text as an extension, and conversely, the reader of this review should also obtain ref. [1] which is freely available on-line, published in open format by the publisher in order to access some of the hard to get references used in this volume.

This immediately takes us to the question that experts in the field will pose: is in this synthesis anything scientifically new? The answer is yes. The list is actually quite long, and the advice is: read, and fill the gaps where the developments stop. Let me point here to one result, the new item which is really not all that new: 1978 [28], Hagedorn and I discovered that hadronic matter with exponential mass spectrum at the point of singularity $T_{\mathrm{H}}$ has a universally vanishing speed of sound $c_{s} \rightarrow 0$ in leading order.

Universally means that this is true for all functions that I have tried, including in particular a variation on preexponential singularity index $a$. In "leading order" means that the most singular parts of both energy density and pressure are considered. This result is found in a report that was submitted to a Bormio Winter Meeting proceedings, chapt. 23 in ref. [1] which today is archived electronically at CERN [28].

The reason that this important result $c_{s} \rightarrow 0$ was not preprinted is that Hagedorn and I were working on a larger manuscript from which this Bormio text was extracted. We did not want to preclude the publication of the large paper. However, at the same time we were developing a new field of physics. The main manuscript was never to be finished and submitted, but the field of physics took off. Thus the Bormio report is all that remains in public view.

This result, $\left.c_{s}\right|_{T \rightarrow T_{\mathrm{H}}} \rightarrow 0$ has other remarkable consequences: Sound velocity that goes to zero at the critical boundary implies that the matter is sticky there, when pressed from inside many unusual things can happen, one being filamenting break-up we call sudden hadronization - the amazing thing is that while I was writing this, Giorgio Torrieri was just reminding me about this insight he had shared before with me. Could $\left.c_{s}\right|_{T \rightarrow T_{\mathrm{H}}} \rightarrow 0$ actually be the cause why the SHM study of the fireball properties obtains such clean sets of results?

Any universal hot hadron matter critical property can be tested with lattice-QCD and the results available do show a range where $\left.c_{s}\right|_{T \rightarrow T_{\mathrm{H}}} \simeq 0$. Thus, the value of $T_{\mathrm{H}}$ may become available as the point of a minimum sound velocity. This criterion comes with the Hagedorn exponential mass spectrum "attached": the result is valid if and when there is an exponential growth in hadron mass spectrum.
In this text I also answer simple questions which turn out to have complicated answers. For example, what is, and when was, QGP discovered? It turns out that QGP as a phrase meant something else initially, and all kinds of variants such as: hot quark matter; hadron plasma, were in use. This makes literature search difficult.

I also tracked the reporting and interviews from the time when CERN decided in February 2000 to step forward with its announcement of the QGP discovery. I learned that the then director of BNL was highly skeptical of the CERN results. And I was shocked to learn that one of the two authors of the CERN scientific consensus report declared a few months down the road that he was mistaken.

Seeing these initial doubts, and being expert on strangeness I thought I ought to take a late deep look at how the signature of CERN February 2000 announcement held up in past 15 years. I am happy to tell that it is doing very well; the case of QGP at CERN-SPS in terms of strange antibaryon signature is very convincing. I hope the reader will join me in this evaluation, seeing the results shown. These are not comprehensive (my apologies) but sufficient to make the point.

I have spent a lot of time, ink, and paper, to explain here why RHI collisions and QGP physics is, was, and remains a frontier of our understanding of physics. It is true that for the trees we sometimes lose the view of the forest. Thus at a few opportunities in this report I went outside the trees to tell how the forest looks today, after 35 years of healthy growth. While some will see my comments as speculative, others may choose to work out the consequences, both in theory and experiment.

\section{Commendation}

I am deeply indebted to Rolf Hagedorn of CERN-TH whose continued mentoring nearly 4 decades ago provided much of the guidance and motivation in my long pursuit of strangeness in quark-gluon plasma and hadronization mechanisms. Rolf Hagedorn was the scientist whose dedicated, determined personal commitment formed the deep roots of this novel area of physics. In 1964/65 Hagedorn proposed the Hagedorn Temperature $T_{\mathrm{H}}$ and the Statistical Bootstrap Model (SBM). These novel ideas opened up the physics of hot hadronic matter to at first, theoretical and later, experimental study, in relativistic heavy ion collision experiments.

I thank (alphabetically) Michael Danos (deceased), Wojciech Florkowski, Marek Gaździcki, Rolf Hagedorn (deceased), Peter Koch, Inga Kuznetsova, Jean Letessier, Berndt Müller, Emanuele Quercigh, Krzysztof Redlich, Helmut Satz, and Giorgio Torrieri, who have all contributed in an essential way to further my understanding of relativistic heavy ion collisions, hot hadronic matter, statistical hadronization, and the strangeness signature of QGP. I thank Tamas Biro for critical comments of a draft manuscript helping to greatly improve the contents; and Victoria Grossack for her kind assistance with the manuscript presentation. I thank the CERN-TH for hospitality in Summer 2015 while this project was created and completed. This work has been in part supported by the US Department of Energy, Office of Science, Office of Nuclear Physics under award number DE-FG02-04ER41318. 
Open Access This is an open access article distributed under the terms of the Creative Commons Attribution License (http://creativecommons.org/licenses/by/4.0), which permits unrestricted use, distribution, and reproduction in any medium, provided the original work is properly cited.

\section{References}

1. J. Rafelski (Editor), Melting Hadrons, Boiling Quarks: From Hagedorn temperature to ultra-relativistic heavyion collisions at CERN; with a tribute to Rolf Hagedorn (Springer, Heidelberg, 2015).

2. P. Koch, B. Müller, J. Rafelski, Phys. Rep. 142, 167 (1986) Strangeness in Relativistic Heavy Ion Collisions.

3. H.C. Eggers, J. Rafelski, Int. J. Mod. Phys. A 6, 1067 (1991) Strangeness and quark gluon plasma: aspects of theory and experiment.

4. J. Rafelski, J. Letessier, A. Tounsi, Acta Phys. Pol. B 27, 1037 (1996) Strange particles from dense hadronic matter, nucl-th/0209080.

5. J. Letessier, J. Rafelski, Int. J. Mod. Phys. E 9, 107 (2000) Observing quark gluon plasma with strange hadrons, nuclth/0003014.

6. J. Letessier, J. Rafelski, Hadrons and quark-gluon plasma, in Cambridge Monographs on Particle Physics Nuclear Physics and Cosmology, Vol. 18 (Cambridge University Press, Cambridge, 2002) pp. 1-397.

7. J. Letessier, J. Rafelski, Eur. Phys. J. A 35, 221 (2008) Hadron production and phase changes in relativistic heavy ion collisions, nucl-th/0504028.

8. S. Weinberg, The Quantum Theory of Fields, Vols. 1, 2 (Cambridge University Press, Cambridge 1995, 1996).

9. Kohsuke Yagi, Tetsuo Hatsuda, Quark-Gluon Plasma: From Big Bang to Little Bang (Cambridge University Press, Cambridge, 2008).

10. J. Rafelski, CERN Cour. 54, 10:57 (2014) http:// cerncourier.com/cws/article/cern/59346, Birth of the Hagedorn temperature.

11. R. Hagedorn, Nuovo Cimento Suppl. 3, 147 (1965) Statistical thermodynamics of strong interactions at highenergies.

12. S.C. Frautschi, Phys. Rev. D 3, 2821 (1971) Statistical bootstrap model of hadrons.

13. R. Hagedorn, Lect. Notes Phys. 221, 53 (1985) preprint CERN-TH-3918/84, reprinted in chapt. 25 of ref. [1] How We Got to QCD Matter From the Hadron Side by Trial, Error.

14. H.G. Pugh, Introductory remarks presented at the Workshop on Future Relativistic Heavy Ion Experiments held at GSI Darmstadt 7-10 October 1980, appeared in proceedings: GSI Orange report 1981-6, edited by R. Bock, R. Stock, preprint LBL-11974 web location verfied July 2015; http://www.osti.gov/scitech/biblio/6639601, Future relativistic heavy ion experiments.

15. J. Rafelski, Eur. Phys. J. A 51, 115 (2015) Originally printed in GSI81-6 Orange Report, pp. 282-324, Extreme States of Nuclear Matter-1980, edited by R. Bock, R. Stock.

16. J. Rafelski, Eur. Phys. J. A 51, 116 (2015) Originally printed in LBL-16281 pp. 489-510, Strangeness and Phase Changes in Hot Hadronic Matter - 1983, also report number UC-34C, DOE CONF-830675, preprint CERN-TH-3685, web location verified July 2015 https://cds.cern.ch/record/147343/ files/198311019.pdf.

17. J. Kapusta, B. Müller, J. Rafelski, Quark-Gluon Plasma: Theoretical Foundations: An annotated reprint collection (Elsevier, Amsterdam, 2003) pp. 1-817.

18. J. Rafelski, Eur. Phys. J. ST 155, 139 (2008) Strangeness Enhancement: Challenges and Successes.

19. J. Yellin, Nucl. Phys. B 52, 583 (1973) An explicit solution of the statistical bootstrap.

20. T.E.O. Ericson, J. Rafelski, CERN Cour. 43N7, 30 (2003) web location verified July 2015 http://cds . cern. ch/record/1733518 The tale of the Hagedorn temperature.

21. C.J. Hamer, S.C. Frautschi, Phys. Rev. D 4, 2125 (1971) Determination of asymptotic parameters in the statistical bootstrap model.

22. S.C. Frautschi, C.J. Hamer, Nuovo Cimento A 13, 645 (1973) Effective temperature of resonance decay in the statistical bootstrap model.

23. C.J. Hamer, Phys. Rev. D 8, 3558 (1973) Explicit solution of the statistical bootstrap model via Laplace transforms.

24. C.J. Hamer, Phys. Rev. D 9, 2512 (1974) Single Cluster Formation in the Statistical Bootstrap Model.

25. R.D. Carlitz, Phys. Rev. D 5, 3231 (1972) Hadronic matter at high density.

26. R. Hagedorn, Nuovo Cimento A 52, 1336 (1967) On the hadronic mass spectrum.

27. W. Nahm, Nucl. Phys. B 45, 525 (1972) Analytical solution of the statistical bootstrap model.

28. J. Rafelski, R. Hagedorn, Thermodynamics of hot nuclear matter in the statistical bootstrap model, presented at Bormio Winter Meeting January 1979, also chapt. 23 in ref. [1], web location verfied July 2015: http:// inspirehep.net/record/1384658/files/CM-P00055555 .pdf.

29. H. Satz, Lect. Notes Phys. 841, 1 (2012). Extreme states of matter in strong interaction physics. An introduction.

30. A. Tounsi, J. Letessier, J. Rafelski, in Proceedings of Divonne 1994 Hot hadronic matter, NATO-ASI series $\mathrm{B}$, Vol. 356, Hadronic matter equation of state and the hadron mass spectrum (1995) pp. 105-116.

31. J. Letessier, J. Rafelski, unpublished, Evaluation made for the CERN Courier article in honor of R. Hagedorn [20], based on the method of ref. [30].

32. M. Beitel, K. Gallmeister, C. Greiner, Phys. Rev. C 90, 045203 (2014) Thermalization of Hadrons via Hagedorn States, arXiv:1402.1458 [hep-ph].

33. A.M. Polyakov, Phys. Lett. B 72, 477 (1978) Thermal Properties of Gauge Fields and Quark Liberation.

34. L. Susskind, Phys. Rev. D 20, 2610 (1979) Lattice Models of Quark Confinement at High Temperature.

35. J.C. Collins, M.J. Perry, Phys. Rev. Lett. 34, 1353 (1975) Superdense Matter: Neutrons Or Asymptotically Free Quarks?

36. N. Cabibbo, G. Parisi, Phys. Lett. B 59, 67 (1975) Exponential Hadronic Spectrum and Quark Liberation.

37. B.C. Barrois, Nucl. Phys. B 129, 390 (1977) Superconducting Quark Matter.

38. M.G. Alford, K. Rajagopal, F. Wilczek, Phys. Lett. B 422, 247 (1998) QCD at finite baryon density: Nucleon droplets and color superconductivity, hep-ph/9711395. 
39. M.G. Alford, K. Rajagopal, F. Wilczek, Nucl. Phys. B 537, 443 (1999) Color flavor locking and chiral symmetry breaking in high density QCD, hep-ph/9804403.

40. M.G. Alford, A. Schmitt, K. Rajagopal, T. Schfer, Rev. Mod. Phys. 80, 1455 (2008) Color superconductivity in dense quark matter, arXiv:0709.4635 [hep-ph].

41. W. Broniowski, W. Florkowski, Phys. Lett. B 490, 223 (2000) Different Hagedorn temperatures for mesons and baryons from experimental mass spectra, compound hadrons, and combinatorial saturation, hep-ph/0004104.

42. W. Broniowski, W. Florkowski, L.Y. Glozman, Phys. Rev. D 70, 117503 (2004) Update of the Hagedorn mass spectrum, hep-ph/0407290.

43. J. Cleymans, D. Worku, Mod. Phys. Lett. A 26, 1197 (2011) The Hagedorn temperature Revisited, arXiv:1103.1463 [hep-ph].

44. T.D. Cohen, V. Krejcirik, J. Phys. G 39, 055001 (2012) Does the Empirical Meson Spectrum Support the Hagedorn Conjecture?, arXiv:1107.2130 [hep-ph].

45. T.S. Biro, A. Peshier, Phys. Lett. B 632, 247 (2006) Limiting temperature from a parton gas with power-law tailed distribution, hep-ph/0506132.

46. P.M. Lo, M. Marczenko, K. Redlich, C. Sasaki, Matching Hagedorn mass spectrum with Lattice QCD, arXiv:1507.06398 [nucl-th].

47. A. Majumder, B. Müller, Phys. Rev. Lett. 105, 252002 (2010) Hadron Mass Spectrum from Lattice QCD, arXiv:1008.1747 [hep-ph].

48. S.Z. Belenky, Nucl. Phys. 2, 259 (1956) Connection between scattering and multiple production of particles.

49. K. Redlich, H. Satz, The Legacy of Rolf Hagedorn: Statistical Bootstrap, Ultimate Temperature, arXiv:1501.07523 [hep-ph], chapt. 7 in [1]

50. J. Rafelski, Nucl. Phys. Proc. Suppl. 243, 155 (2013) Connecting QGP-Heavy Ion Physics to the Early Universe, arXiv:1306.2471 [astro-ph.CO].

51. L.P. Csernai, J.I. Kapusta, Phys. Rev. D 46, 1379 (1992) Nucleation of relativistic first order phase transitions.

52. T.S. Biro, A. Jakovac, Phys. Rev. D 90, 094029 (2014) $Q C D$ above $T_{c}$ : Hadrons, partons, and the continuum, arXiv:1405.5471 [hep-ph].

53. A.Z. Mekjian, Phys. Rev. C 17, 1051 (1978) Explosive nucleosynthesis, equilibrium thermodynamics, and relativistic heavy-ion collisions.

54. I. Montvay, J. Zimanyi, Nucl. Phys. A 316, 490 (1979) Hadron Chemistry in Heavy Ion Collisions.

55. P. Koch, J. Rafelski, Nucl. Phys. A 444, 678 (1985) Time Evolution of Strange Particle Densities in Hot Hadronic Matter.

56. H. Koppe, Phys. Rev. 76, 688 (1949) On the Production of Mesons.

57. H. Koppe, Z. Naturforsch. A 3, 251 (1948) The meson output from the bombardment of light nuclei with $\alpha$ particles.

58. E. Fermi, Prog. Theor. Phys. 5, 570 (1950) High-energy nuclear events.

59. I.Y. Pomeranchuk, Dokl. Akad. Nauk Ser. Fiz. 78, 889 (1951) (translated in [17], pp. 20-23) On the theory of multiple particle production in a single collision.

60. R. Hagedorn, Nuovo Cimento A 56, 1027 (1968) Hadronic matter near the boiling point.

61. P. Koch, J. Rafelski, South Afr. J. Phys. 9, 8 (1986) web locatio verified July 2015 http://www.physics. arizona.edu/ ${ }^{\sim}$ rafelski/Books/85SAJPKocRaf.pdf Why the hadronic gas description of hadronic reactions works: The example of strange hadrons.

62. G. Torrieri, J. Rafelski, New J. Phys. 3, 12 (2001) Search for QGP and thermal freezeout of strange hadrons, hep$\mathrm{ph} / 0012102$.

63. W. Broniowski, W. Florkowski, Phys. Rev. C 65, 064905 (2002) Strange particle production at RHIC in a single freezeout model, nucl-th/0112043.

64. A. Baran, W. Broniowski, W. Florkowski, Acta Phys. Pol. B 35, 779 (2004) Description of the particle ratios and transverse momentum spectra for various centralities at RHIC in a single freezeout model, nucl-th/0305075.

65. J. Rafelski, Phys. Lett. B 262, 333 (1991) Strange antibaryons from quark - gluon plasma.

66. T. Csorgo, L.P. Csernai, Phys. Lett. B 333, 494 (1994) Quark - gluon plasma freezeout from a supercooled state?, hep-ph/9406365.

67. L.P. Csernai, I.N. Mishustin, Phys. Rev. Lett. 74, 5005 (1995) Fast hadronization of supercooled quark - gluon plasma.

68. T.S. Biro, P. Levai, J. Zimanyi, Phys. Rev. C 59, 1574 (1999) Hadronization with a confining equation of state, hep-ph/9807303.

69. J. Rafelski, J. Letessier, Phys. Rev. Lett. 85, 4695 (2000) Sudden hadronization in relativistic nuclear collisions, hep-ph/0006200.

70. A. Keranen, J. Manninen, L.P. Csernai, V. Magas, Phys. Rev. C 67, 034905 (2003) Statistical hadronization of supercooled quark gluon plasma, nucl-th/0205019.

71. S. Borsanyi, Nucl. Phys. A 904, 270c (2013) Thermodynamics of the QCD transition from lattice, arXiv: 1210.6901 [hep-lat], presented at the 23rd International Conference on Ultrarelativistic Nucleus-Nucleus Collisions (QM 2012), August 13-18, 2012, Washington, DC, USA.

72. S. Borsanyi, Z. Fodor, C. Hoelbling, S.D. Katz, S. Krieg, K.K. Szabo, Phys. Lett. B 730, 99 (2014) Full result for the $Q C D$ equation of state with $2+1$ flavors, arXiv:1309.5258 [hep-lat].

73. M. Petran, J. Letessier, V. Petracek, J. Rafelski, Phys. Rev. C 88, 034907 (2013) Hadron production and quark-gluon plasma hadronization in $\mathrm{Pb}-\mathrm{Pb}$ collisions at $\sqrt{s_{N N}}=2.76 \mathrm{TeV}$, arXiv:1303.2098 [hep-ph].

74. M. Petran, J. Rafelski, Phys. Rev. C 88, 021901 (2013) Universal hadronization condition in heavy ion collisions at $\sqrt{s_{\mathrm{NN}}}=62 \mathrm{GeV}$ and at $\sqrt{s_{\mathrm{NN}}}=2.76 \mathrm{TeV}$, arXiv:1303.0913 [hep-ph].

75. M. Petran, J. Letessier, V. Petracek, J. Rafelski, J. Phys. Conf. Ser. 509, 012018 (2014) Interpretation of strange hadron production at LHC, arXiv:1309.6382 [hep-ph].

76. M. Petran, J. Letessier, V. Petracek, J. Rafelski, Acta Phys. Pol. Suppl. 5, 255 (2012) Strangeness Production in Au-Au collisions at $\sqrt{s_{N N}}=62.4 \mathrm{GeV}$, arXiv:1112.3189 [hep-ph].

77. J. Rafelski, J. Letessier, J. Phys. G 36, 064017 (2009) Critical Hadronization Pressure, arXiv:0902.0063 [hep$\mathrm{ph}$.

78. J. Rafelski, J. Letessier, PoS CONFINEMENT 8, 111 (2008) Particle Production and Deconfinement Threshold, arXiv:0901.2406 [hep-ph].

79. J. Letessier, J. Rafelski, Phys. Rev. C 59, 947 (1999) Chemical non-equilibrium and deconfinement in 200A/GeV sulphur induced reactions, hep-ph/9806386. 
80. P. Braun-Munzinger, J. Stachel, J.P. Wessels, N. Xu, Phys. Lett. B 344, 43 (1995) Thermal equilibration and expansion in nucleus-nucleus collisions at the AGS, nuclth/9410026.

81. A. Andronic, P. Braun-Munzinger, J. Stachel, Nucl. Phys. A 772, 167 (2006) Hadron production in central nucleus-nucleus collisions at chemical freeze-out, nuclth/0511071.

82. F. Becattini, P. Castorina, A. Milov, H. Satz, Eur. Phys. J. C 66, 377 (2010) A Comparative analysis of statistical hadron production, arXiv:0911.3026 [hep-ph].

83. J. Manninen, F. Becattini, Phys. Rev. C 78, 054901 (2008) Chemical freeze-out in ultra-relativistic heavy ion collisions at $\sqrt{s_{\mathrm{NN}}}=130$ and 200-GeV, arXiv:0806.4100 [nucl-th].

84. F. Becattini, M. Gazdzicki, A. Keranen, J. Manninen, R. Stock, Phys. Rev. C 69, 024905 (2004) Chemical equilibrium in nucleus nucleus collisions at relativistic energies, hep-ph/0310049.

85. J. Cleymans, H. Oeschler, K. Redlich, S. Wheaton, Phys. Rev. C 73, 034905 (2006) Comparison of chemical freezeout criteria in heavy-ion collisions, hep-ph/0511094.

86. STAR Collaboration (B.I. Abelev et al.), Phys. Rev. C 79, 034909 (2009) Systematic Measurements of Identified Particle Spectra in pp, $d^{+} A u$ and $A u+A u$ Collisions from STAR, arXiv:0808.2041 [nucl-ex].

87. ALICE Collaboration (B. Abelev et al.), Phys. Rev. Lett. 109, 252301 (2012) Pion, Kaon, and Proton Production in Central $\mathrm{Pb}-\mathrm{Pb}$ Collisions at $\sqrt{s_{N N}}=2.76 \mathrm{TeV}$, arXiv:1208.1974 [hep-ex].

88. ALICE Collaboration (B. Abelev et al.), Phys. Rev. C 88, 044910 (2013) Centrality dependence of $\pi, K, p$ production in $\mathrm{Pb}-\mathrm{Pb}$ collisions at $\sqrt{s_{N N}}=2.76 \mathrm{TeV}$, arXiv:1303.0737 [hep-ex].

89. I.A. Karpenko, Y.M. Sinyukov, K. Werner, Phys. Rev. C 87, 024914 (2013) Uniform description of bulk observables in the hydrokinetic model of $A+A$ collisions at the BNL Relativistic Heavy Ion Collider and the CERN Large Hadron Collider, arXiv:1204.5351 [nucl-th].

90. A.S. Botvina, J. Steinheimer, E. Bratkovskaya, M. Bleicher, J. Pochodzalla, Phys. Lett. B 742, 7 (2015) Formation of hypermatter and hypernuclei within transport models in relativistic ion collisions, arXiv:1412.6665 [nucl-th].

91. J. Letessier, A. Tounsi, U.W. Heinz, J. Sollfrank, J. Rafelski, Phys. Rev. D 51, 3408 (1995) Strangeness conservation in hot nuclear fireballs, hep-ph/9212210.

92. J. Rafelski, J. Letessier, Nucl. Phys. A 715, 98 (2003) Testing limits of statistical hadronization, nuclth/0209084.

93. T.D. Lee, Trans. N.Y. Acad. Sci., Ser. II 40, 111 (1980); Based on September 28, 1979 lecture at Columbia University; Preprint CU-TP-170 (1979) Is the Physical Vacuum a Medium?, reprinted in: T.D. Lee, Selected papers, edited by G. Feinberg, Vol. 2 (Birkhauser, Boston, 1986) pp. 213-225.

94. T.D. Lee, Particle Physics and Introduction to Field Theory (Science Press, Harwood Academic, Beijing, Chur, London, 1981).

95. K.G. Wilson, Phys. Rev. D 10, 2445 (1974) Confinement of Quarks.

96. A. Chodos, R.L. Jaffe, K. Johnson, C.B. Thorn, V.F. Weisskopf, Phys. Rev. D 9, 3471 (1974) A New Extended Model of Hadrons.
97. T.A. DeGrand, R.L. Jaffe, K. Johnson, J.E. Kiskis, Phys. Rev. D 12, 2060 (1975) Masses and Other Parameters of the Light Hadrons.

98. K. Johnson, Acta Phys. Pol. B 6, 865 (1975) The M.I.T. Bag Model.

99. A.W. Thomas, Adv. Nucl. Phys. 13, 1 (1984) Chiral Symmetry and the Bag Model: A New Starting Point for Nuclear Physics.

100. A.T.M. Aerts, J. Rafelski, Phys. Lett. B 148, 337 (1984) QCD, Bags and Hadron Masses.

101. A.T.M. Aerts, J. Rafelski, Strange Hadrons In The Mit Bag Model CERN-TH-4160-85, UCT-TP-27-21985, web location verified July 2015 http://www-lib. kek.jp/cgi-bin/img_index?8505459.

102. R.L. Thews, M. Schroedter, J. Rafelski, Phys. Rev. C 63, 054905 (2001) Enhanced $J / \psi$ production in deconfined quark matter, hep-ph/0007323.

103. M. Schroedter, R.L. Thews, J. Rafelski, Phys. Rev. C 62, 024905 (2000) $B_{c}$ meson production in nuclear collisions at RHIC, hep-ph/0004041.

104. A. Rothkopf, T. Hatsuda, S. Sasaki, Phys. Rev. Lett. 108, 162001 (2012) Complex Heavy-Quark Potential at Finite Temperature from Lattice QCD, arXiv:1108.1579 [hep-lat].

105. A. Bazavov, P. Petreczky, Nucl. Phys. A 904,599c (2013) On static quark anti-quark potential at non-zero temperature, arXiv:1210.6314 [hep-lat].

106. B.J. Harrington, A. Yildiz, Phys. Rev. Lett. 33, 324 (1974) High Density Phase Transitions in Gauge Theories.

107. S.A. Chin, Phys. Lett. B 78, 552 (1978) Transition to Hot Quark Matter in Relativistic Heavy Ion Collision.

108. A. Bazavov et al., Phys. Rev. D 80, 014504 (2009) Equation of state and QCD transition at finite temperature, arXiv:0903.4379 [hep-lat].

109. HotQCD Collaboration (A. Bazavov et al.), Phys. Rev. D 90, 094503 (2014) Equation of state in $(2+1)$-flavor $Q C D$, arXiv:1407.6387 [hep-lat].

110. J. Letessier, J. Rafelski, Phys. Rev. C 67, 031902 (2003) QCD equations of state and the QGP liquid model, hep$\mathrm{ph} / 0301099$.

111. J.O. Andersen, N. Haque, M.G. Mustafa, M. Strickland, N. Su, Equation of State for QCD at finite temperature and density. Resummation versus lattice data, arXiv:1411.1253 [hep-ph], invited talk at 11th Quark Confinement and the Hadron Spectrum, September 8-12 2014, Saint Petersburg, Russia.

112. M. Strickland, J.O. Andersen, A. Bandyopadhyay, N. Haque, M.G. Mustafa, N. Su, Nucl. Phys. A 931, 841 (2014) Three loop HTL perturbation theory at finite temperature and chemical potential, arXiv:1407.3671.

113. N. Haque, A. Bandyopadhyay, J.O. Andersen, M.G. Mustafa, M. Strickland, N. Su, JHEP 05, 027 (2014) Three-loop HTLpt thermodynamics at finite temperature and chemical potential, arXiv:1402.6907 [hep-ph]

114. L. Van Hove, in 17th International Symposium on Multiparticle Dynamics, held in Seewinkel, Austria, 16-20 Jun 1986, edited by J. MacNaughton, W. Majerotto, Walter, M. Markytan (World Scientific, Singapore, 1986) pp. 801818 Theoretical prediction of a new state of matter, the "quark-gluon plasma" (also called "quark matter").

115. E.V. Shuryak, Phys. Lett. B 78, 150 (1978) (Sov. J. Nucl. Phys. 28, 408 (1978)) (Yad. Fiz. 28, 796 (1978)) Quark- 
Gluon Plasma and Hadronic Production of Leptons, Photons and Psions.

116. O.K. Kalashnikov, V.V. Klimov, Phys. Lett. B 88, 328 (1979) Phase Transition in Quark-Gluon Plasma.

117. D.D. Ivanenko, D.F. Kurdgelaidze, Astrophysics 1, 251 (1965) (Astrofiz. 1, 479 (1965)) Hypothesis concerning quark stars.

118. H. Fritzsch, M. Gell-Mann, H. Leutwyler, Phys. Lett. B 47, 365 (1973) Advantages of the Color Octet Gluon Picture.

119. P. Carruthers, Collect. Phenomena 1, 147 (1974) Quarkium: a bizarre Fermi liquid.

120. A.D. Linde, Rep. Prog. Phys. 42, 389 (1979) Phase Transitions in Gauge Theories and Cosmology.

121. B.A. Freedman, L.D. McLerran, Phys. Rev. D 16, 1169 (1977) Fermions and Gauge Vector Mesons at Finite Temperature and Density. 3. The Ground State Energy of a Relativistic Quark Gas.

122. E.V. Shuryak, Sov. Phys. JETP 47, 212 (1978) (Zh. Eksp. Teor. Fiz. 74, 408 (1978)) Theory of Hadronic Plasma.

123. J.I. Kapusta, Nucl. Phys. B 148, 461 (1979) Quantum Chromodynamics at High Temperature.

124. E.V. Shuryak, Phys. Rep. 61, 71 (1980) Quantum Chromodynamics and the Theory of Superdense Matter.

125. G.F. Chapline, A.K. Kerman, Preprint CTP-695 MITCambridge - April (1978) web location verified July 2015 http://inspirehep.net/record/134446/files/CTP695.pdf On the Possibility of Making Quark Matter in Nuclear Collisions.

126. G.F. Chapline, M.H. Johnson, E. Teller, M.S. Weiss, Phys. Rev. D 8, 4302 (1973) Highly excited nuclear matter.

127. L. Lederman, J. Weneser (Editors), Workshop on BeV/nucleon collisions of heavy ions: How and Why held at Bear Mountain, New York, November 29-December 1, 1974, proceedings available as Brookhaven National Laboratory Report \#50445, (BNL-50445, Upton, NY, 1975), web location verified July 2015 http: //www.osti. gov/scitech/servlets/purl/4061527.

128. T.D. Lee, G.C. Wick, Phys. Rev. D 9, 2291 (1974) Vacuum stability and vacuum excitation in a spin-0 field theory.

129. G. Odyniec, Begin of the search for the Quark-Gluon Plasma, chapt. 12 in ref. [1]

130. H.H. Gutbrod, The Path to Heavy Ions at LHC and Beyond, chapt. 13 in ref. [1].

131. S.A. Chin, A.K. Kerman, Phys. Rev. Lett. 43 , 1292 (1979) Possible longlived hyperstrange multi-quark droplets.

132. G.F. Chapline, A. Granik, Nucl. Phys. A 459, 681 (1986) Production of quark matter via oblique shock waves.

133. R. Anishetty, P. Koehler, L.D. McLerran, Phys. Rev. D 22, 2793 (1980) Central collisions between heavy nuclei at extremely high-energies: The fragmentation region.

134. R. Hagedorn, CERN-TH-3014 (1980), reprinted in chapt. 26 of ref. [1] How To Deal With Relativistic Heavy Ion Collisions.

135. J.D. Bjorken, Phys. Rev. D 27, 140 (1983) Highly Relativistic Nucleus-Nucleus Collisions: The Central Rapidity Region.

136. M. Danos, J. Rafelski, Heavy Ion Phys. 14, 97 (2001) Baryon rich quark gluon plasma in nuclear collision, nuclth/0011049 Formation of quark-gluon plasma at central rapidity (This manuscript is identical with University of Cape Town preprint UCT-TP $7 / 84$ which is the long version of University of Frankfurt preprint UFTP-82/94).

137. R. Hagedorn, I. Montvay, J. Rafelski, Thermodynamics Of Nuclear Matter From The Statistical Bootstrap Model, CERN-TH-2605, in Hadronic Matter, Erice, October 1978, edited by N. Cabibbo, L. Sertorio (Plenum Press, New York, 1980) p. 49.

138. CERN press release, web location verfied July 2015 http://press.web.cern.ch/press-releases/2000/02/ new-state-matter-created-cern.

139. Brookhaven National Laboratory, (2000, February 10), Tantalizing Hints Of Quark Gluon Plasma Found At CERN ScienceDaily, web location verfied July 2015 www . sciencedaily.com/releases/2000/02/000209215728. htm.

140. BRAHMS Collaboration (I. Arsene et al.), Nucl. Phys. A 757, 1 (2005) Quark gluon plasma and color glass condensate at RHIC? The Perspective from the BRAHMS experiment, nucl-ex/0410020.

141. PHENIX Collaboration (K. Adcox et al.), Nucl. Phys. A 757, 184 (2005) Formation of dense partonic matter in relativistic nucleus-nucleus collisions at RHIC: Experimental evaluation by the PHENIX collaboration, nuclex/0410003.

142. PHOBOS Collaboration (B.B. Back et al.), Nucl. Phys. A 757, 28 (2005) The PHOBOS perspective on discoveries at $R H I C$, nucl-ex/0410022.

143. STAR Collaboration (J. Adams et al.), Nucl. Phys. A 757, 102 (2005) Experimental and theoretical challenges in the search for the quark gluon plasma: The STAR Collaboration's critical assessment of the evidence from RHIC collisions, nucl-ex/0501009.

144. BNL press release, web location verfied July 2015 https://www . bnl.gov/rhic/news2/news . asp?a=5756/\& $\mathrm{t}=$ today.

145. J. Rafelski, B. Müller, Phys. Rev. Lett. 48, 1066 (1982) 56, 2334(E) (1986) Strangeness production in the QuarkGluon Plasma.

146. T. Biro, J. Zimanyi, Phys. Lett. B 113, 6 (1982) Quarkochemistry in Relativistic Heavy Ion Collisions.

147. J. Rafelski, Phys. Rep. 88, 331 (1982) Formation and Observables of the Quark-Gluon Plasma.

148. J. Rafelski, Strangeness in Quark - Gluon Plasma, lecture at Quark Matter Formation And Heavy Ion Collisions, Bielefeld, May 1982, due to mail mishap ultimately published in South Afr. J. Phys. 6, 37 (1983), see chapts. 30, 31 in ref. [1].

149. L. Van Hove, Quark Matter Formation And Heavy Ion Collisions: The Theoretical Situation, preprint CERNTH-3360 19 July 1982, in Multiparticle Dynamics 1982 Volendam 6-11 June 1982; edited by W. Kittel, W. Metzger, A. Stergiou (World Scientific, Singapore, 1983) pp. 244-266,

150. F. Antinori, The heavy ion physics programme at the CERN OMEGA spectrometer, web location verfied July 2015 http://cds.cern.ch/record/343249/files/p43. pdf pp. 43-49 in edited by M. Jacob, E. Quercigh, CERN-Yellow Report 97-02, Symposium on the CERN Omega Spectrometer: 25 Years of Physics held 19 March 1997, CERN Geneva, Switzerland, web location verfied July 2015 http://cds.cern.ch/record/330556/ files/CERN-97-02.pdf. 
151. WA97 Collaboration (E. Andersen et al.), Phys. Lett. B 449, 401 (1999) Strangeness enhancement at mid-rapidity in $\mathrm{Pb} \mathrm{Pb}$ collisions at $158-\mathrm{A}-\mathrm{GeV} / \mathrm{c}$.

152. STAR Collaboration (B. Abelev et al.), Phys. Lett. B 673, 183 (2009) Energy and system size dependence of phi meson production in $\mathrm{Cu}+\mathrm{Cu}$ and $\mathrm{Au}+\mathrm{Au}$ collisions, arXiv:0810.4979 [nucl-ex].

153. ALICE Collaboration (B. Abelev et al.), Phys. Lett. B 728, 216 (2014) 734, 409(E) (2014) Multi-strange baryon production at mid-rapidity in $\mathrm{Pb}-\mathrm{Pb}$ collisions at $\sqrt{s_{N N}}=$ $2.76 \mathrm{TeV}$, arXiv:1307.5543 [nucl-ex].

154. K. Geiger, D. Kumar Srivastava, Phys. Rev. C 56, 2718 (1997) Parton cascade description of relativistic heavy ion collisions at CERN SPS energies?, nucl-th/9706002.

155. K. Geiger, Phys. Rep. 258, 237 (1995) Space-time description of ultrarelativistic nuclear collisions in the $Q C D$ parton picture.

156. H.J. Drescher, M. Hladik, S. Ostapchenko, T. Pierog, K. Werner, Phys. Rep. 350, 93 (2001) Parton based GribovRegge theory, hep-ph/0007198.

157. E. Iancu, R. Venugopalan, The Color glass condensate and high-energy scattering in QCD, Quark gluon plasma 3 in edited by R.C. Hwa, X.-N. Wang, (World Scientific, Singapore, 2003) pp. 249-336 [hep-ph/0303204].

158. K. Geiger, Phys. Rev. D 46, 4965 (1992) Thermalization in ultrarelativistic nuclear collisions. 1. Parton kinetics and quark gluon plasma formation.

159. K. Geiger, Phys. Rev. D 46, 4986 (1992) Thermalization in ultrarelativistic nuclear collisions. 2. Entropy production and energy densities at RHIC and LHC.

160. B. Müller, A. Schafer, Int. J. Mod. Phys. E 20, 2235 (2011) Entropy Creation in Relativistic Heavy Ion Collisions, arXiv:1110.2378 [hep-ph].

161. A. Kurkela, E. Lu, Phys. Rev. Lett. 113, 182301 (2014) Approach to Equilibrium in Weakly Coupled Non-Abelian Plasmas, arXiv:1405.6318 [hep-ph].

162. L. Labun, J. Rafelski, Acta Phys. Pol. B 41, 2763 (2010) Strong Field Physics: Probing Critical Acceleration and Inertia with Laser Pulses and Quark-Gluon Plasma, arXiv:1010.1970 [hep-ph].

163. J. Letessier, J. Rafelski, A. Tounsi, Phys. Rev. C 50, 406 (1994) Gluon production, cooling and entropy in nuclear collisions, hep-ph/9711346.

164. J. Letessier, J. Rafelski, A. Tounsi, Acta Phys. Pol. A 85 , 699 (1994) In search of entropy.

165. B. Müller, Phys. Rev. C 67, 061901 (2003) Phenomenology of jet quenching in heavy ion collisions, nuclth/0208038.

166. A. Majumder, M. Van Leeuwen, Prog. Part. Nucl. Phys. A 66, 41 (2011) The Theory and Phenomenology of Perturbative QCD Based Jet Quenching, arXiv:1002.2206 [hep-ph].

167. L. Bhattacharya, R. Ryblewski, M. Strickland, Photon production from a non-equilibrium quark-gluon plasma, arXiv:1507.06605 [hep-ph].

168. R. Ryblewski, M. Strickland, Phys. Rev. D 92, 025026 (2015) Dilepton production from the quark-gluon plasma using $(3+1)$-dimensional anisotropic dissipative hydrodynamics, arXiv:1501.03418 [nucl-th].

169. V. Petousis, Theoretical Review on $Q C D$ and Vector Mesons in Dileptonic Quark Gluon Plasma, arXiv: 1208.4437 [hep-ph].

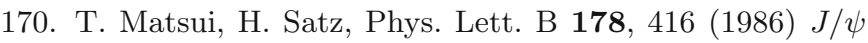
Suppression by Quark-Gluon Plasma Formation.

171. A. Andronic, Nucl. Phys. A 931, 135 (2014) Experimental results and phenomenology of quarkonium production in relativistic nuclear collisions, arXiv:1409.5778 [nucl-ex].

172. J.P. Blaizot, D. De Boni, P. Faccioli, G. Garberoglio, Heavy quark bound states in a quark-gluon plasma: dissociation and recombination, arXiv:1503.03857 [nucl-th].

173. ALICE Collaboration (B.B. Abelev et al.), Phys. Lett. B 734, 314 (2014) Centrality, rapidity and transverse momentum dependence of $\mathrm{J} / \psi$ suppression in $\mathrm{Pb}-\mathrm{Pb}$ collisions at $\sqrt{s_{\mathrm{NN}}}=2.76 \mathrm{TeV}$, arXiv:1311.0214 [nucl-ex].

174. PHENIX Collaboration (A. Adare et al.), Systematic study of charged-pion and kaon femtoscopy in Au+Au collisions at $\sqrt{s_{N N}}=200 \mathrm{GeV}$, arXiv:1504.05168 [nucl-ex].

175. ALICE Collaboration (J. Adam et al.), One-dimensional pion, kaon, and proton femtoscopy in $\mathrm{Pb}-\mathrm{Pb}$ collisions at $\sqrt{s_{\mathrm{NN}}}=2.76 \mathrm{TeV}$, arXiv:1506.07884 [nucl-ex]

176. T. Altinoluk, N. Armesto, G. Beuf, A. Kovner, M. Lublinsky, Bose enhancement and the ridge, arXiv:1503.07126 [hep-ph].

177. L.P. Csernai, G. Mocanu, Z. Neda, Phys. Rev. C 85, 068201 (2012) Fluctuations in Hadronizing QGP, arXiv:1204.6394 [nucl-th].

178. A. Wroblewski, Acta Phys. Pol. B 16, 379 (1985) On The Strange Quark Suppression Factor In High-energy Collisions.

179. K. Rajagopal, Nucl. Phys. A 661, 150 (1999) Mapping the QCD phase diagram, hep-ph/9908360.

180. M. Gazdzicki, M. Gorenstein, P. Seyboth, Acta Phys. Pol. B 42, 307 (2011) Onset of deconfinement in nucleusnucleus collisions: Review for pedestrians and experts, arXiv:1006.1765 [hep-ph].

181. NA49 Collaboration (K. Grebieszkow), Acta Phys. Pol. B 43, 609 (2012) Report from NA49, arXiv:1112.0794 [nuclex].

182. NA61/SHINE Collaboration (N. Abgrall et al.), Report from the NA61/SHINE experiment at the CERN SPS CERN-SPSC-2014-031, SPSC-SR-145 (2014), web location verified July 2015 http://cds . cern.ch/record/ 1955138.

183. NA61/SHINE Collaboration (M. Gazdzicki), EPJ Web of Conference 95, 01005 (2015) Recent results from NA61/SHINE, arXiv:1412.4243 [hep-ex].

184. P. Sorensen, J. Phys. Conf. Ser. 446, 012015 (2013) Beam Energy Scan Results from RHIC.

185. STAR Collaboration (N.R. Sahoo), J. Phys. Conf. Ser. 535, 012007 (2014) Recent results on event-by-event fluctuations from the RHIC Beam Energy Scan program in the STAR experiment, arXiv:1407.1554 [nucl-ex].

186. S. Durr, Z. Fodor, J. Frison, C. Hoelbling, R. Hoffmann, S.D. Katz, S. Krieg, T. Kurth et al., Science 322, 1224 (2008) Ab-Initio Determination of Light Hadron Masses, arXiv:0906.3599 [hep-lat].

187. A.S. Kronfeld, Lattice Gauge Theory and the Origin of Mass, in 100 Years of Subatomic Physics, edited by E.M. Henley, S.D. Ellis (World Scientific, Singapore, 2013) pp. 493-518 arXiv:1209.3468 [physics.hist-ph].

188. S. Aoki et al., Eur. Phys. J. C 74, 2890 (2014) Review of lattice results concerning low-energy particle physics, arXiv:1310.8555 [hep-lat].

189. A. Di Giacomo, H.G. Dosch, V.I. Shevchenko, Y.A. Simonov, Phys. Rep. 372, 319 (2002) Field correlators in QCD: Theory and applications, hep-ph/0007223. 
190. A. Einstein, Aether, the theory of relativity reprinted in The Berlin Years Writings 1918-1921, Aether and the Theory of Relativity edited by M. Janssen, R. Schulmann, J. Illy, Ch. Lehner, D.K. Buchwald, see pp. 305-309; and p. 321 (Document 38, Collected Papers of Albert Einstein, Princeton University Press, 2002), English version translation by the author, see also translation in A. Einstein Sidelights on Relativity (Dover Publishers, 1983) ondemand 2015.

191. M.J. Fromerth, J. Rafelski, Hadronization of the quark Universe, astro-ph/0211346.

192. The Alpha Magnetic Spectrometer: What For web location verfied July 2015 http://www.ams02.org/whatis-ams/what-for/.

193. J. Birrell, J. Rafelski, Phys. Lett. B 741, 77 (2015) Quarkgluon plasma as the possible source of cosmological dark radiation, arXiv:1404.6005 [nucl-th].

194. J. Rafelski, J. Birrell, J. Phys. Conf. Ser. 509, 012014 (2014) Traveling Through the Universe: Back in Time to the Quark-Gluon Plasma Era, arXiv:1311.0075 [nucl-th].

195. W. Heisenberg, Z. Phys. 101, 533 (1936) Zur Theorie der "Schauer" in der Höhenstrahlung.

196. H. Satz, Phys. Rev. D 20, 582 (1979) Dimensionality in the Statistical Bootstrap Model.

197. K. Redlich, L. Turko, Z. Phys. C 5, 201 (1980) Phase Transitions in the Statistical Bootstrap Model with an Internal Symmetry.

198. R. Hagedorn, J. Rafelski, Phys. Lett. B 97, 136 (1980) Hot Hadronic Matter and Nuclear Collisions.

199. K. Zalewski, K. Redlich, Thermodynamics of the low density excluded volume hadron gas, arXiv:1507.05433 [hep$\mathrm{ph}$.

200. V. Vovchenko, D.V. Anchishkin, M.I. Gorenstein, Phys. Rev. C 91, 024905 (2015) Hadron Resonance Gas Equation of State from Lattice $Q C D$, arXiv:1412.5478 [nuclth].

201. B. Touschek, Nuovo Cimento B 58, 295 (1968) Covariant statistical mechanics.

202. J.I. Kapusta, Phys. Rev. D 23, 2444 (1981) Asymptotic Mass Spectrum and Thermodynamics of the Abelian Bag Model.

203. J.I. Kapusta, Nucl. Phys. B 196, 1 (1982) Asymptotic Level Density of Constrained and Interacting Fields.

204. K. Redlich, Z. Phys. C 21, 69 (1983) Asymptotic Hadron Mass Spectrum With an Internal Non-abelian Symmetry Group.

205. M.I. Gorenstein, V.K. Petrov, G.M. Zinovev, Phys. Lett. B 106, 327 (1981) Phase Transition in the Hadron Gas Model.

206. M.I. Gorenstein, M. Gazdzicki, W. Greiner, Phys. Rev. C 72, 024909 (2005) Critical line at the deconfinement phase transition.

207. G. Torrieri, S. Steinke, W. Broniowski, W. Florkowski, J. Letessier, J. Rafelski, Comput. Phys. Commun. 167, 229 (2005) SHARE: Statistical hadronization with resonances, nucl-th/0404083.

208. G. Torrieri, S. Jeon, J. Letessier, J. Rafelski, Comput. Phys. Commun. 175, 635 (2006) SHAREv2: Fluctuations and a comprehensive treatment of decay feed-down, nuclth/0603026.

209. M. Petran, J. Letessier, J. Rafelski, G. Torrieri, Comput. Phys. Commun. 185, 2056 (2014) SHARE with CHARM, arXiv: 1310.5108 [hep-ph].
210. Alberica Toia, CERN Cour. 53N4, 31 (2013) Participants and spectators at the heavy-ion fireball.

211. ALICE Collaboration (B. Abelev et al.), Phys. Rev. C 88 , 044909 (2013) Centrality determination of $\mathrm{Pb}-\mathrm{Pb}$ collisions at $\sqrt{s_{N N}}=2.76 \mathrm{TeV}$ with ALICE, arXiv:1301.4361 [nucl-ex].

212. J.W. Li, D.S. Du, Phys. Rev. D 78, 074030 (2008) The Study of $\mathrm{B} \rightarrow J / \psi \eta^{\prime}$ decays and determination of $\eta \rightarrow \eta^{\prime}$ mixing angle, arXiv:0707.2631 [hep-ph].

213. V. Begun, W. Florkowski, M. Rybczynski, Phys. Rev. C 90, 014906 (2014) Explanation of hadron transversemomentum spectra in heavy-ion collisions at $\sqrt{s_{N N}}=$ $2.76 \mathrm{TeV}$ within chemical non-equilibrium statistical hadronization model, arXiv:1312.1487 [nucl-th].

214. V. Begun, W. Florkowski, M. Rybczynski, Phys. Rev. C 90, 054912 (2014) Transverse-momentum spectra of strange particles produced in $\mathrm{Pb}+\mathrm{Pb}$ collisions at $\sqrt{s_{\mathrm{NN}}}=2.76 \mathrm{TeV}$ in the chemical non-equilibrium model, arXiv:1405.7252 [hep-ph].

215. ALICE Collaboration (B. Abelev), Strangeness with $A L$ ICE: from $p p$ to $\mathrm{Pb}-\mathrm{Pb}$, arXiv:1209.3285 [nucl-ex], Proceedings of The Physics of the LHC 2012, held 4-9 June 2012 in Vancouver, BC, http://plhc2012.triumf.ca/.

216. ALICE Collaboration (B. Abelev et al.), Phys. Rev. Lett. 111, 222301 (2013) $K_{S}^{0}$ and $\Lambda$ production in $\mathrm{Pb}-\mathrm{Pb}$ collisions at $\sqrt{s_{N N}}=2.76 \mathrm{TeV}$, arXiv:1307.5530 [nucl-ex].

217. I. Kuznetsova, J. Rafelski, Eur. Phys. J. C 51, 113 (2007) Heavy flavor hadrons in statistical hadronization of strangeness-rich QGP, hep-ph/0607203.

218. P. Braun-Munzinger, D. Magestro, K. Redlich, J. Stachel, Phys. Lett. B 518, 41 (2001) Hadron production in Au-Au collisions at RHIC, hep-ph/0105229.

219. F. Karsch, Nucl. Phys. A 698, 199 (2002) Lattice results on QCD thermodynamics, hep-ph/0103314.

220. F. Karsch, Nucl. Phys. Proc. Suppl. 83, 14 (2000) Lattice QCD at finite temperature and density, hep-lat/9909006.

221. J. Rafelski, J. Letessier, Phys. Rev. C 83, 054909 (2011) Particle Production in $s_{\mathrm{NN}}=2.76 \mathrm{TeV}$ Heavy Ion Collisions, arXiv:1012.1649 [hep-ph].

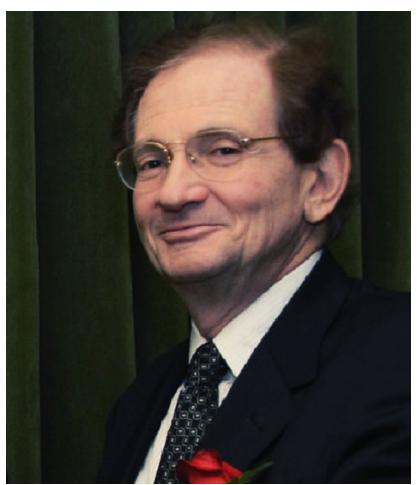

Johann Rafelski received his $\mathrm{PhD}$ in 1973, addressing the physics of Strong Fields with Walter Greiner in Frankfurt. In 1977 he arrived at CERN, where in collaboration with Rolf Hagedorn he turned to the study of Relativistic Heavy Ion Collisions and Quark Gluon Plasma (QGP). He continued this work in Frankfurt, CapeTown and finally, Arizona, where he has been since 1987 . Rafelski's efforts on strangeness signature of QGP guided a wide experimental effort at CERN-SPS, leading to the announcement of the QGP discovery in the year 2000. During the last 15 years he worked to understand strange and charm QGP data obtained at BNLRHIC and CERN-LHC. 